\title{
Introdução à teoria de álgebras e módulos conformais
}

\author{
Renato Alessandro Martins
}

\author{
DISSERTAÇÃO APRESENTADA \\ Instituto DE MATEMÁTICA E EstatísticA \\ UNIVERSIDADE DE SÃo PAUlO \\ PARA \\ OBTENÇÂO DO TÍTULO \\ $\mathrm{DE}$ \\ Mestre EM CIÊNCIAS
}

Área de Concentração: Matemática

Orientador: Prof. Dr. Vyacheslav Futorny

Durante o desenvolvimento deste trabalho o autor recebeu auxílio financeiro do $\mathrm{CNPq}$

São Paulo, julho de 2008 


\section{Introdução à teoria de álgebras e módulos conformais}

Este exemplar corresponde à redação final da dissertação devidamente corrigida e defendida por Renato Alessandro Martins e aprovada pela Comissão Julgadora.

Banca Examinadora:

- Prof. Dr. Vyacheslav Futorny (orientador) - IME-USP.

- Prof. Dr. Luis Antônio Peresi - IME-USP.

- Prof. Dr. Adriano Adrega de Moura - IMECC-UNICAMP. 


\section{Agradecimentos}

Agradeço primeiramente aos meus pais por me apoiarem durante toda a minha caminhada até a conclusão deste mestrado.

E também aos meus amigos Rodrigo Ferraz, Rodrigo Roque, Ana Carolina e Mariana Smit por estarem sempre ao meu lado nos piores, e também nos melhores momentos deste curso.

Agradeço a ajuda e compreensão de todos os amigos que fui fazendo durante este curso (como o Samir, o Gustavo, o Oscar, entre outros) e também aos que tinha na graduação mas que infelizmente não consegui manter o mesmo contato agora (como o Ulysses, a Giselle Bertaglia, entre outros). Vocês foram pessoas muito importantes na minha trajetória.

Também sou grato aos bons professores deste instituto que, de alguma forma, me ajudaram a chegar até aqui, seja de forma indireta (como fizeram Maria Cristina Bonomi Barufi e Heloísa Daruiz Borsari, através de seus excelentes cursos que me motivaram a seguir esta carreira) ou de forma mais direta (como fez José Antônio Verderesi, me ajudando num momento muito difćil, mesmo sem nunca ter sido, de fato, meu professor).

A todos aqueles que torceram por mim, meus sinceros agradecimentos. 


\section{Resumo}

O Capítulo 1 tem o objetivo de familiarizar o leitor com a linguagem de distribuições formais introduzindo a função formal delta, a expansão de uma distribuição formal e a transformada formal de Fourier.

Já no Capítulo 2, veremos o conceito de superálgebras de Lie de distribuições formais, localidade entre duas distribuições formais e suas equivalências, além do conceito de $O P E$. Também apresentamos muitos exemplos de superálgebras de Lie e de superálgebras de Lie de distribuições formais, devido à sua importância nesta teoria.

No Capítulo 3, definiremos superálgebra conformal (ou conforme) e o associaremos às superálgebras de Lie de distribuições formais através de uma "equivalência" entre estas fazendo uso da teoria de Categorias. A partir daí, começamos a analisar o problema da classificação de superálgebras conformes finitas e irredutíveis apresentando três exemplos de superálgebras conformes e enunciando o teorema de $[D K]$ que os utiliza.

No quarto Capítulo, classificaremos os módulos conformes finitos e irredutíveis, apresentando as definições, propriedades, e exemplos básicos que nos permitem iniciar tal estudo.

Palavras-chave: superálgebra conforme, módulo conforme, distribuições formais. 


\section{Abstract}

In Chapter 1, we present the language of formal distributions, introducing the formal delta function, the concept of formal distribution expansion and the Fourier Formal Transform.

In Chapter 2, we study formal distributions of Lie superalgebras, locality of formal distributions and its equivalences, the concept of $O P E$ and examples of Lie superalgebras and formal distributions of Lie superalgebras.

In Chapter 3, we define conformal superalgebras and associate them with formal distributions of Lie superalgebras using category theory, which provide us the grounds to study the classification problem of finite irreducible Lie superalgebras, and we present three examples of conformal superalgebras and a $[D K]$ theorem using them.

In Chapter 4, we classify finite irreducible conformal modules, presenting definitions, properties and basic examples which allow us to begin the study of this subject.

Keywords: conformal superalgebras, conformal modules, formal distributions. 


\section{Sumário}

1 Distribuições Formais $\quad 1$

1.1 Introdução . . . . . . . . . . . . . . . . . . . 1

1.2 Função Formal Delta . . . . . . . . . . . . . . . . . . . . 3

1.3 Expansão de uma distribuição formal . . . . . . . . . . . . . . 11

1.4 A Transformada Formal de Fourier . . . . . . . . . . . . . . 19

2 Superálgebras de Lie de distribuições formais $\quad 26$

2.1 Introdução . . . . . . . . . . . . . . . . . . . 26

2.2 O conceito de localidade de duas distribuições formais numa superálgebra de Lie . . . . . . . . . . . . . . . . 28

$2.3 \mathrm{O} \lambda$-produto e o $\lambda$-colchete de duas distribuições formais . . . 34

2.4 Fórmula de Taylor . . . . . . . . . . . . . . . . . . 41

2.5 Exemplos de superálgebras de Lie . . . . . . . . . . . . 43

2.5.1 Álgebras de Witt . . . . . . . . . . . . . . . . . . . 44

2.5.2 Extensões centrais . . . . . . . . . . . . . . . . 44

2.5.3 Álgebras de Heisenberg . . . . . . . . . . . . . . . 45

2.5.4 A álgebra de Virasoro . . . . . . . . . . . . . . . 46

2.5.5 O afinizador de Kac-Moody . . . . . . . . . . . . 47

2.5.6 Afinizador de Clifford . . . . . . . . . . . . . . . 48

2.5.7 Álgebras de Correntes . . . . . . . . . . . . . . . . 48

2.5.8 Álgebra de Neveu-Schwarz . . . . . . . . . . . . . . . . 49

2.6 Exemplos de superálgebras de Lie de distribuições formais . . 49

2.7 Pesos Conformes . . . . . . . . . . . . . . . . . . 54

3 Superálgebras Conformes $\quad 66$

3.1 Introdução . . . . . . . . . . . . . . . . . 66

3.2 A equivalência entre a categoria das superálgebras conformes e a categoria das classes de equivalência das superálgebras de Lie de distribuições formais . . . . . . . . . . . . . . . . . . 81

3.3 Álgebra de Vertex . . . . . . . . . . . . . . . . . . . . . 93

4 Módulos Conformes $\quad 98$

4.1 Introdução . . . . . . . . . . . . . . . . . . . . . . . . 99 
4.2 Classificação dos módulos conformes finitos irredutíveis . . . . 112 


\section{Introdução}

As álgebras de Vertex vêm sendo, nas últimas décadas, uma importante ferramenta em diversos assuntos da Física e da Matemática como, por exemplo, na representação de álgebras de dimensão infinita e na teoria de grupos finitos. Apenas para comentar, uma álgebra de Vertex é, basicamente, um espaço $V$ onde a cada elemento se corresponde uma distribuição formal. A primeira definição histórica foi dada em [6] sendo muito complexa, mas a atual, apresentada na Seção 3, é definida como tendo duas estruturas que se relacionam:

- a de uma superálgebra de Lie conforme

- a de uma superálgebra diferencial simétrica à esquerda (que comentaremos posteriormente)

As (super)álgebras conformes (ou conformais), a qual definiremos no capítulo 3, possuem uma conexão fascinante com os Hamiltonianos na teoria das "equações não-lineares evolucionárias". De fato, a primeira aparição na literatura de estruturas do tipo conforme antecedeu a descoberta das álgebras de Vertex e chegou ao Cálculo de Variações [13]. A teoria das álgebras conformes tem sido muito utilizada na tentativa de resolver o problema da classificação de álgebras de Lie de dimensão infinita. Cerca de 40 anos atrás, Victor G. Kac propôs (e parcialmente resolveu) o problema da classificação das álgebras de Lie simples $\mathbb{Z}$-graduadas de Gelfand-Kirillov de dimensão finita (ver [9]). Este problema foi completamente resolvido por Mathieu em $[10]-[12]$.

Quanto aos módulos conformes, pode-se dizer que se trata de uma ferramenta básica para a construção de realizações de campos livres de superálgebras de Lie de dimensão infinita na teoria de campos conformes. Esta é uma das razões para se classificar e construir tais módulos. Neste trabalho, resolve-se tal problema através da suposição de irredutibilidade da álgebra de Virasoro e de Neveu-Schwarz, das superálgebras de Correntes e suas somas semidiretas (ver Capítulo 4).

Antes de começar o trabalho, é importante ressaltar que a idéia principal 
do mesmo será utilizar três linguagens "equivalentes". A primeira será a linguagem das distribuições formais locais (ver Capítulo 2), a segunda será a linguagem de módulos sobre as superálgebras conformes (ver Capítulo 4) e a terceira e última será a linguagem dos módulos conformes sobre a subálgebra aniquiladora estendida, a qual nos permitirá classificar os módulos conformes finitos e irredutíveis através dos cruciais Teorema 4.21 e Lema 4.22.

Em vista a tudo isso, aborda-se no Capítulo 1 todo o conteúdo necessário para iniciar o estudo das distribuições formais mutuamente locais, o que será efetivamente feito no Capítulo 2.

No Capítulo 3, ao invés de expor a teoria de módulos conformes (o que somente será feito no Capítulo 4), trabalha-se com a teoria de superálgebras conformes devido à sua importância, à sua complexidade, e também à quantidade de resultados expostos, os quais destacamos a classificação das superálgebras conformes finitas e irredutíveis, cuja solução pode ser vista em [4].

Finalmente, o Capítulo 4 nos apresenta as duas últimas linguagens com as quais o resultado sobre módulos conformes é obtido. Para sua leitura, é necessário o conhecimento desenvolvido nos Capítulos anteriores, sendo imprescindível a leitura de todo o Capítulo 3, e não somente a dos dois primeiros.

O leitor interessado pode consultar [1] e [2] para obter maiores detalhes sobre o assunto abordado no Capítulo 1. Quanto ao Capítulo 2 recomendo [1] e [5], para o Capítulo 3 as referências [1] e [4] e, finalmente, recomendo [3] para o Capítulo [4].

Desta forma, comecemos nosso trabalho. 


\section{Capítulo 1}

\section{Distribuições Formais}

\subsection{Introdução}

Este Capítulo tem o objetivo de familiarizar o leitor com a linguagem de distribuições formais, fornecendo o conhecimento necessário para utilizar esta teoria nos capítulos subseqüêntes. Com este objetivo, introduz-se inicialmente as definições e propriedades básicas e inicia-se a Seção $1.2 \mathrm{com}$ a função formal delta $\delta(z-w)$, que será muito utilizada em toda esta dissertação. Em seguida, trabalha-se com as expansões de distribuições formais, onde define-se o conceito de localidade de uma distribuição formal e apresenta-se o conceito de expansão $O P E$ de uma distribuição formal $a(z, w)$. Na Seção 1.4, estuda-se a transformada formal de Fourier devido à sua importância futura no Capítulo 2 para a introdução do conceito de $\lambda$-produto, e também no Capítulo 3 , em que introduz-se o conceito de $\lambda$ colchete como uma ferramenta bastante útil nesta teoria.

Sendo assim, tem-se a seguinte definição de distribuição formal:

Definição 1.1. Seja $U$ um espaço vetorial sobre $\mathbb{C}$. Chamaremos de distribuição formal nas indeterminadas $z_{1}, z_{2}, \ldots, z_{n}$ com valores em $U$ às séries da forma:

$$
a\left(z_{1}, z_{2}, \ldots, z_{n}\right)=\sum_{i_{1}, \ldots, i_{n}=1}^{\infty} a_{i_{1}, \ldots, i_{n}} \cdot z_{1}^{i_{1}} \cdot \ldots \cdot z_{n}^{i_{n}}
$$

onde $a_{i_{1}, \ldots, i_{n}} \in U$ para todo $i_{1}, \ldots, i_{n} \in \mathbb{Z}$.

Estas distribuições formais formam um espaço vetorial sobre $\mathbb{C}$ que será denotado por $U\left[\left[z_{1}, z_{1}^{-1}, \ldots, z_{n}, z_{n}^{-1}\right]\right]$ (aqui a utilização dos dois colchetes é necessária, já que utiliza-se um colchete apenas quando o número de 
termos for finito. Por exemplo, $\left.a(z) \in \mathbb{C}\left[z^{-1}, z\right]\right]$ significa que $a(z)$ é uma distribuição formal com quantidade finita de potências negativas). Nem sempre duas distribuições formais podem ser multiplicadas. Para tal propósito, é necessário verificar sua convergência no sentido algébrico, isto é, se os coeficientes de cada monômio $z_{1}^{i_{1}} \ldots z_{n}^{i_{n}}$ será uma soma convergente. Por exemplo, quando uma das distribuições formais é, na verdade, um polinômio de Laurent, então o produto estará bem definido, já que cada coeficiente $a_{i_{1}, \ldots, i_{n}}$ é obtido por uma soma finita, logo convergente.

Definição 1.2. Seja $a(z)=\sum_{n \in \mathbb{Z}} a_{n} z^{n} \in U\left[\left[z, z^{-1}\right]\right]$. Define-se o resíduo de $a(z)$, e o denota-se por $\operatorname{Res}_{z} a(z)$, como o coeficiente $a_{-1}$ de $a(z)$, isto é:

$$
\operatorname{Res}_{z} a(z)=a_{-1}
$$

e também seja $\partial a(z)$ a derivada de a(z) na variável $z$

$$
\partial a(z)=\sum_{n \in \mathbb{Z}} n \cdot a_{n} z^{n-1}
$$

Assim, quando o produto $a(z) b(z)$ está bem definido, tem-se a seguinte igualdade:

$$
\operatorname{Res}_{z} \partial a(z) b(z)=-\operatorname{Res}_{z} a(z) \partial b(z)
$$

onde $\partial a(z) b(z)$ significa $(\partial a(z)) \cdot b(z)$ e $\operatorname{Res}_{z} a(z) b(z)$ significa $\operatorname{Res}_{z}(a(z)$. $b(z))$. De fato, como $\partial(a(z) b(z))=\partial a(z) b(z)+a(z) \partial b(z)$, e $\operatorname{Res}_{z} \partial c(z)=0$, tem-se que:

$$
\begin{aligned}
0 & =\operatorname{Res}_{z} \partial(a(z) b(z)) \\
& =\operatorname{Res}_{z}(\partial a(z) b(z)+a(z) \partial b(z)) \\
& =\operatorname{Res}_{z} \partial a(z) b(z)+\operatorname{Res}_{z} a(z) \partial b(z)
\end{aligned}
$$

Denotando a álgebra dos polinômios de Laurent em $z$ por $\mathbb{C}\left[z, z^{-1}\right]$, temse a seguinte definição:

Definição 1.3. Sejam $f(z) \in U\left[\left[z, z^{-1}\right]\right]$ e $\varphi(z) \in \mathbb{C}\left[z, z^{-1}\right]$. Defina a seguinte função: 


$$
\begin{aligned}
& \langle\cdot, \cdot\rangle: U\left[\left[z, z^{-1}\right]\right] \times \mathbb{C}\left[z, z^{-1}\right] \quad \longrightarrow \quad U \\
& (f, \varphi) \quad \longmapsto \operatorname{Res}_{z} f(z) \varphi(z)
\end{aligned}
$$

O polinômio $\varphi(z) \in \mathbb{C}\left[z, z^{-1}\right]$ é chamado, neste caso, de função de teste. Vale o seguinte teorema:

Teorema 1.4. Sejam $a(z), b(z) \in U\left[\left[z, z^{-1}\right]\right]$. Então as distribuições formais a $(z)$ e $b(z)$ são iguais, se e somente se, para toda função de teste $\varphi(z) \in \mathbb{C}\left[z, z^{-1}\right]$, valer que $\langle a, \varphi\rangle=\langle b, \varphi\rangle$ (onde $a=a(z)$ e $b=b(z)$ ).

Demonstração. De fato, $a(z)=b(z)$ implica que $\langle a, \varphi\rangle=\langle b, \varphi\rangle$. Assim, basta provar que $\langle a, \varphi\rangle=\langle b, \varphi\rangle$, para todo $\varphi$, implica que $a(z)=b(z)$. Fixado $m$, defina $\varphi_{m}=z^{m-1}$. Então:

$$
\begin{aligned}
\left\langle a, \varphi_{m}\right\rangle & =\operatorname{Res}_{z} a(z) \varphi_{m}(z) \\
& =\operatorname{Res}_{z} a(z) \\
& =\sum_{n \in \mathbb{Z}} a_{n} \cdot z^{n} \cdot z^{m-1} \\
& =a_{m}
\end{aligned}
$$

Analogamente, temos que $\left\langle b, \varphi_{m}\right\rangle=b_{m}$. Logo $a_{m}=b_{m}$, para todo $m \in \mathbb{N}$, e $\operatorname{assim} a(z)=b(z)$.

\subsection{Função Formal Delta}

Como já foi comentado no início do capítulo, uma das distribuições formais merece atenção especial devido a sua importância na teoria. Trata-se da função formal delta $\delta(z-w) \in U\left[\left[z, z^{-1}, w, w^{-1}\right]\right]$ definida por:

$$
\delta(z-w)=z^{-1} \sum_{n \in \mathbb{Z}}\left(\frac{w}{z}\right)^{n}=\sum_{n \in \mathbb{Z}} w^{n} z^{-n-1}
$$


A função formal delta possui algumas propriedades importantes que serão demonstradas através da seguinte construção:

Seja $C=\mathbb{C}\left[\left[z, z^{-1}, w, w^{-1}\right]\right]$ e seja $S=(z-w)^{n}$ tal que $n \geq 0$. O anel de frações de $C$ com respeito à $S$ (denotado por $S^{-1} C$ ) consiste das funções racionais da forma

$$
\frac{f(z, w)}{(z-w)^{n}}
$$

onde $f=f(z, w) \in C$ e $n \geq 0$. As derivações $\partial_{z}$ e $\partial_{w}$ agem naturalmente neste anel de acordo com a regra do quociente para diferenciações. Por exemplo:

$$
\partial_{z} \frac{f(z, w)}{(z-w)^{n}}=\frac{\partial_{z} f(z, w) \cdot(z-w)-n \cdot f(z, w)}{(z-w)^{n+1}}
$$

Denote $i_{z, w}$ e $i_{w, z}$ como a inclusão em $C$ das séries de potência dos anéis $\mathbb{C}\left[\left[\frac{w}{z}\right]\right]$ e $\mathbb{C}\left[\left[\frac{z}{w}\right]\right]$, respectivamente. Observando que:

$$
(z-w) \cdot z^{-1} \cdot\left(1+\frac{w}{z}+\ldots+\left(\frac{w}{z}\right)^{k}+\ldots\right)=1 \text { em } \mathbb{C}\left[\left[\frac{w}{z}\right]\right]
$$

e que

$$
(z-w) \cdot z^{-1} \cdot\left(1+\frac{z}{w}+\ldots+\left(\frac{z}{w}\right)^{k}+\ldots\right)=1 \text { em } \mathbb{C}\left[\left[\frac{z}{w}\right]\right]
$$

tem-se então que:

$$
\begin{aligned}
i_{z, w} \frac{1}{z-w} & =z^{-1} \cdot \sum_{m=0}^{\infty}\left(\frac{w}{z}\right)^{m}=\sum_{m=0}^{\infty} z^{-m-1} w^{m} \\
i_{w, z} \frac{1}{z-w} & =-w^{-1} \cdot \sum_{m=0}^{\infty}\left(\frac{z}{w}\right)^{m}=-\sum_{m=0}^{\infty} z^{m} w^{-m-1} \\
& =-\sum_{m=-1}^{-\infty} z^{-m-1} w^{m}
\end{aligned}
$$


Usando indução, prova-se que:

$$
i_{z, w} \frac{1}{(z-w)^{j+1}}=\sum_{m=0}^{\infty}\left(\begin{array}{c}
m \\
j
\end{array}\right) z^{-m-1} w^{m-j}
$$

e também que

$$
i_{w, z} \frac{1}{(z-w)^{j+1}}=-\sum_{m=-1}^{-\infty}\left(\begin{array}{c}
m \\
j
\end{array}\right) z^{-m-1} w^{m-j}
$$

O que foi feito em (1.7) é o seguinte:

$$
i_{z, w}: \mathbb{C}\left[\left[\frac{w}{z}\right]\right] \longrightarrow C \text { é tal que } \frac{f(z, w)}{(z-w)^{n}} \longmapsto f(z, w) \cdot(z-w)^{-n}
$$

Assim, tem-se uma série de potências $f(z, w)$ em $\mathbb{C}\left[\left[\frac{w}{z}\right]\right]$ e como $i_{z, w}$ é apenas a inclusão em $C$, segue que:

$$
\begin{aligned}
i_{z, w}\left(\frac{1}{z-w}\right) & =i_{z, w}\left(z^{-1} \cdot \sum_{m=0}^{\infty}\left(\frac{w}{z}\right)^{m}\right) \\
& =i_{z, w} \sum_{m=0}^{\infty} w^{m} z^{-m-1} \\
& =\sum_{m=0}^{\infty} z^{-m-1} w^{m}
\end{aligned}
$$

Para facilitar os cálculos, pode-se utilizar o seguinte argumento (menos formal, porém mais utilizado):

$$
\begin{aligned}
i_{z, w} \frac{1}{z-w} & =\frac{1}{z} \cdot \frac{1}{1-\frac{w}{z}} \\
& =z^{-1} \cdot \sum_{m=0}^{\infty}\left(\frac{w}{z}\right)^{m} \\
& =\sum_{m=0}^{\infty} z^{-m-1} w^{m}
\end{aligned}
$$


Para finalizar, tem-se que tanto $i_{z, w}$ quanto $i_{w, z}$ comutam com $\partial_{z}$ e com $\partial_{w}$. Por exemplo:

$$
\begin{aligned}
i_{z, w} \partial_{z} \frac{f(z, w)}{(z-w)^{n}} & =i_{z, w} \frac{\left(\partial_{z} f(z, w)\right) \cdot(z-w)-n \cdot f(z, w)}{(z-w)^{n+1}} \\
& =\left(\partial_{z} f\right) \cdot(z-w)^{-n}-n \cdot(z-w)^{-(n+1)} f \\
& =\partial_{z}\left(f \cdot(z-w)^{-n}\right) \\
& =\partial_{z} i_{z, w} \frac{f}{(z-w)^{n}}
\end{aligned}
$$

Em vista desta construção, prova-se as propriedades da função delta abaixo:

Teorema 1.5. São verdadeiras as seguintes afirmações:

(i) $\partial_{w}^{(j)} \delta(z-w)=i_{z, w} \frac{1}{(z-w)^{j+1}}-i_{w, z} \frac{1}{(z-w)^{j+1}}=\sum_{m=0}^{\infty}\left(\begin{array}{c}m \\ j\end{array}\right) z^{-m-1} w^{m-j}$ onde $A^{(j)}=\frac{A^{j}}{j !}$, para todo operador $A$.

(ii) Para toda distribuição formal $f(z) \in U\left[\left[z, z^{-1}\right]\right]$, tem-se que:

$$
\operatorname{Res}_{z} f(z) \delta(z-w)=f(w)
$$

(iii) $\delta(z-w)=\delta(w-z)$

(iv) $\partial_{z} \delta(z-w)=-\partial_{w} \delta(z-w)$

$(v)(z-w) \cdot \partial_{w}^{(j+1)} \delta(z-w)=\partial_{w}^{(j)} \delta(z-w), \forall j \in \mathbb{Z}$.

(vi) $(z-w)^{j+1} \cdot \delta_{w}^{(j)} \delta(z-w)=0, \forall j \in \mathbb{Z}_{+}$.

(vii) $\delta(z-w) a(z)=\delta(z-w) a(w)$, onde $a(z) \in U\left[\left[z, z^{-1}\right]\right]$.

(viii) $\delta(z-t) \delta(w-t)=\delta(w-z) \delta(z-w)$ 
$(i x) \partial_{t}^{n} \delta(z-t)\left(-\partial_{t}\right)^{m} \delta(w-t)=\sum_{j=0}^{m}\left(\begin{array}{c}m \\ j\end{array}\right) \partial_{w}^{m-j} \delta(w-t) \partial_{w}^{n+j} \delta(z-w)$

Demonstração. Tem-se que:

$$
\begin{aligned}
& i_{z, w} \frac{1}{(z-w)^{j+1}}-i_{w, z} \frac{1}{(z-w)^{j+1}} \\
& =\sum_{m=0}^{\infty}\left(\begin{array}{c}
m \\
j
\end{array}\right) z^{-m-1} w^{m-j}-\left(-\sum_{m=-1}^{-\infty}\left(\begin{array}{c}
m \\
j
\end{array}\right) z^{-m-1} w^{m-j}\right) \\
& =\sum_{m=-\infty}^{\infty}\left(\begin{array}{c}
m \\
j
\end{array}\right) z^{-m-1} w^{m-j}
\end{aligned}
$$

Por outro lado,

$$
\partial_{w}^{(j)} \delta(z-w)=\sum_{m=-\infty}^{\infty}\left(\begin{array}{c}
m \\
j
\end{array}\right) z^{-m-1} w^{m-j}
$$

De fato, o resultado é válido para $j=0$, pois

$$
\begin{aligned}
\delta(z-w) & =\sum_{m \in \mathbb{Z}} z^{-m-1} w^{m} \\
& =\sum_{m=-\infty}^{\infty}\left(\begin{array}{c}
m \\
0
\end{array}\right) z^{-m-1} w^{m-0}
\end{aligned}
$$

Agora suponha o resultado válido para $n<j$. Então tem-se para $n=j$ :

$$
\begin{aligned}
\partial_{w}^{(j)} \delta(z-w) & =\frac{\partial_{w}^{j} \delta(z-w)}{j !} \\
& =\frac{1}{j} \cdot \partial_{w}\left(\partial_{w}^{(j-1)} \delta(z-w)\right) \\
& \stackrel{(1)}{=} \frac{1}{j} \cdot \partial_{w}\left(\sum_{m=-\infty}^{\infty}\left(\begin{array}{c}
m \\
j-1
\end{array}\right) z^{-m-1} w^{m-j+1}\right) \\
& =\sum_{m=-\infty}^{\infty} \frac{m-j+1}{j} \cdot\left(\begin{array}{c}
m \\
j-1
\end{array}\right) z^{-m-1} w^{m-j} \\
& =\sum_{m=-\infty}^{\infty}\left(\begin{array}{c}
m \\
j
\end{array}\right) z^{-m-1} w^{m-j}
\end{aligned}
$$

onde (1) vale por hipótese de indução. Assim provou-se (i). Agora fixe $n \in \mathbb{N}$. Tem-se: 


$$
\begin{aligned}
\operatorname{Res}_{z} z^{n} \delta(z-w) & =\operatorname{Res}_{z} \sum_{m \in \mathbb{N}} w^{m} z^{n-m-1} \\
& =w^{n}
\end{aligned}
$$

Logo, dado $f(z)=\sum_{m \in \mathbb{N}} f_{m} z^{m}$, tem-se:

$$
\begin{aligned}
\operatorname{Res}_{z} f(z) \delta(z-w) & =\operatorname{Res}_{z} \sum_{m \in \mathbb{N}} f_{m} z^{m} \delta(z-w) \\
& =\sum_{m \in \mathbb{N}} f_{m}\left(\operatorname{Res}_{z} z^{m} \delta(z-w)\right) \\
& =\sum_{m \in \mathbb{N}} f_{m} w^{m} \\
& =f(w)
\end{aligned}
$$

donde segue (ii). Agora

$$
\begin{aligned}
\delta(z-w) & =\sum_{n \in \mathbb{Z}} w^{n} z^{-n-1} \\
& \stackrel{(2)}{=} \sum_{m \in \mathbb{Z}} w^{-m-1} z^{m} \\
& =\delta(w-z)
\end{aligned}
$$

e também

$$
\begin{aligned}
\partial_{z} \delta(z-w) & =\partial_{z}\left(\sum_{m \in \mathbb{Z}} w^{m} z^{-m-1}\right) \\
& =\sum_{m \in \mathbb{Z}}(-m-1) \cdot w^{m} z^{-m-2} \\
& \stackrel{(3)}{=} \sum_{m \in \mathbb{Z}}-m \cdot z^{-m-1} w^{m-1} \\
& =-\sum_{m \in \mathbb{Z}} m \cdot z^{-m-1} w^{m-1} \\
& =-\partial_{w} \delta(z-w)
\end{aligned}
$$

onde (2) vale bastando trocar $m$ por $-n-1$ e também vale (3) bastando trocar $m+1$ por $m$. Isto prova (iii) e (iv). Agora 


$$
\begin{aligned}
& (z-w) \cdot \partial_{w}^{(j+1)} \delta(z-w)=(z-w) \cdot \sum_{m=-\infty}^{\infty}\left(\begin{array}{c}
m \\
j+1
\end{array}\right) z^{-m-1} w^{m-j-1} \\
& =\sum_{m=-\infty}^{\infty}\left(\begin{array}{c}
m \\
j+1
\end{array}\right) z^{-m} w^{m-j-1}-\sum_{m=-\infty}^{\infty}\left(\begin{array}{c}
m \\
j+1
\end{array}\right) z^{-m-1} w^{m-j} \\
& \stackrel{(4)}{=} \sum_{n=-\infty}^{\infty}\left(\begin{array}{c}
n+1 \\
j+1
\end{array}\right) z^{-n-1} w^{n-j}-\sum_{n=-\infty}^{\infty}\left(\begin{array}{c}
n \\
j+1
\end{array}\right) z^{-n-1} w^{n-j} \\
& =\sum_{n=-\infty}^{\infty}\left(\begin{array}{c}
n \\
j
\end{array}\right) z^{-n-1} w^{n-j} \\
& =\partial_{w}^{(j)} \delta(z-w)
\end{aligned}
$$

onde (4) vale trocando $m$ por $n+1$ no primeiro somatório e $m$ por $n$ no segundo somatório. Isto prova $(v)$. Para provar $(v i)$, será usado $(v)$. Desta forma, tem-se que:

$$
\begin{aligned}
(z-w)^{j+1} \cdot \partial_{w}^{(j)} \delta(z-w) & =(z-w) \cdot \delta(z-w) \\
& =(z-w) \cdot \sum_{n \in \mathbb{Z}} z^{n} w^{-n-1} \\
& =\sum_{n \in \mathbb{Z}} z^{n+1} w^{-n-1}-\sum_{n \in \mathbb{Z}} z^{n} w^{-n} \\
& =\sum_{m \in \mathbb{Z}} z^{m} w^{-m}-\sum_{m \in \mathbb{Z}} z^{m} w^{-m} \\
& =0
\end{aligned}
$$

Seja $\varphi(z) \in \mathbb{C}\left[z, z^{-1}\right]$ uma função de teste qualquer. Temos:

$$
\begin{aligned}
\langle\partial(z-w) a(z), \varphi(z)\rangle & =\operatorname{Res}_{z} \partial(z-w) a(z) \varphi(z) \\
& =\operatorname{Res}_{z} a(z) \varphi(z) \partial(z-w) \\
& =a(w) \varphi(w) \\
& =a(w) \operatorname{Res}_{z} \varphi(z) \delta(z-w) \\
& =a(w) \operatorname{Res}_{z} \delta(z-w) \varphi(z) \\
& =a(w) \cdot\langle\delta(z-w), \varphi(z)\rangle \\
& =\langle\delta(z-w) a(w), \varphi(z)\rangle
\end{aligned}
$$


Do teorema 1.1., segue que $\delta(z-w) \cdot a(z)=\delta(z-w) \cdot a(w)$, e assim está provado (vii).

Observação: A segunda igualdade é verdadeira, já que todos os coeficientes $a_{m, n}$ de $z^{m} w^{n}$ são iguais a 1 ou a 0 em $\delta(z-w)$. Logo $\delta(z-w)$ comuta com $a(z) \varphi(z)$.

Tratando $z$ como uma constante (definindo $a(t)=\delta(z-t)$ ), tem-se que:

$$
\begin{aligned}
\delta(z-w) \delta(w-t) & =a(t) \delta(w-t) \\
& =\delta(w-t) a(t) \\
& =\delta(w-t) a(w) \\
& =\delta(w-t) \delta(z-w)
\end{aligned}
$$

o que prova (viii). Finalmente, tem-se que:

$$
\begin{aligned}
\partial_{z}^{n} \partial_{w}^{m} \delta(z-t) \delta(w-t) & =\partial_{z}^{n}\left(\delta(z-t) \partial_{w}^{m} \delta(w-t)\right) \\
& =\partial_{z}^{n} \delta(z-t) \cdot \partial_{w}^{m} \delta(w-t) \\
& =\left(-\partial_{t}\right)^{n} \delta(z-t) \cdot\left(-\partial_{t}\right)^{m} \delta(w-t) \\
& =(-1)^{n} \partial_{t}^{n} \delta(z-t) \cdot\left(-\partial_{t}\right)^{m} \delta(w-t)
\end{aligned}
$$

E, por outro lado:

$$
\begin{aligned}
\partial_{z}^{n} \partial_{w}^{m} \delta(w-t) \delta(z-w) & =\partial_{z}^{n}\left(\sum_{j=0}^{m}\left(\begin{array}{c}
m \\
j
\end{array}\right) \partial_{w}^{m-j} \delta(w-t) \cdot \partial_{w}^{j} \delta(z-w)\right) \\
& =\sum_{j=0}^{m}\left(\begin{array}{c}
m \\
j
\end{array}\right) \partial_{w}^{m-j} \delta(w-t) \cdot \partial_{w}^{j} \partial_{z}^{n} \delta(z-w) \\
& =\sum_{j=0}^{m}\left(\begin{array}{c}
m \\
j
\end{array}\right) \partial_{w}^{m-j} \delta(w-t) \cdot \partial_{w}^{j}\left(-\partial_{w}^{n}\right) \delta(z-w) \\
& =(-1)^{n} \sum_{j=0}^{m}\left(\begin{array}{c}
m \\
j
\end{array}\right) \partial_{w}^{m-j} \delta(w-t) \cdot \partial_{w}^{n+j} \delta(z-w)
\end{aligned}
$$


Como $\delta(z-t) \delta(w-t)=\delta(w-t) \delta(z-w)$, segue que:

$\partial_{z}^{n} \partial_{w}^{m} \delta(z-t) \delta(w-t)=\partial_{z}^{n} \partial_{w}^{m} \delta(w-t) \delta(z-w)$

logo vale (ix), pois:

$(-1)^{n} \partial_{t}^{n} \delta(z-t) \cdot\left(-\partial_{t}\right)^{m} \delta(w-t)=(-1)^{n} \sum_{j=0}^{m}\left(\begin{array}{c}m \\ j\end{array}\right) \partial_{w}^{m-j} \delta(w-t) \cdot \partial_{w}^{n+j} \delta(z-w)$

\subsection{Expansão de uma distribuição formal}

Nesta seção o objetivo é analisar quando uma distribuição formal

$$
a(z, w) \in U\left[\left[z, z^{-1}, w, w^{-1}\right]\right]
$$

tem uma expansão da forma:

$$
a(z, w)=\sum_{j=0}^{\infty} c^{j}(w) \cdot \partial_{w}^{(j)} \delta(z-w)
$$

onde $c^{j}(w) \in U\left[\left[w, w^{-1}\right]\right]$.

Teorema 1.6. Seja $a(z, w) \in U\left[\left[z, z^{-1}, w, w^{-1}\right]\right]$ uma distribuição formal que tem uma expansão $\sum_{j=0}^{\infty} c^{j}(w) \cdot \partial_{w}^{(j)} \delta(z-w)$. Então:

$$
c^{n}(w)=\operatorname{Res}_{z}\left(a(z, w) \cdot(z-w)^{n}\right), \forall n \in \mathbb{N} .
$$

Demonstração. De fato: 


$$
\begin{aligned}
\operatorname{Res}_{z} a(z, w) \cdot(z-w)^{n} & =\operatorname{Res}_{z}\left(\sum_{j=0}^{\infty} c^{j}(w)(z-w)^{n} \partial_{w}^{(j)} \delta(z-w)\right) \\
& \stackrel{(1)}{=} \operatorname{Res}_{z}\left(\sum_{j=n}^{\infty} c^{j}(w) \partial_{w}^{(j-n)} \delta(z-w)\right) \\
& \stackrel{(2)}{=} \sum_{j=n}^{\infty} c^{j}(w)\left(\begin{array}{c}
0 \\
j-n
\end{array}\right) w^{-j+n} \\
& =c^{n}(w)+\sum_{j=n+1}^{\infty} c^{j}(w) \cdot 0 \cdot w^{-j+n} \\
& =c^{n}(w)
\end{aligned}
$$

\section{Observações:}

(1) Pelo Teorema 1.1, tem-se que $(z-w)^{n} \partial_{w}^{(j)} \delta(z-w)=\partial_{w}^{(j-n)} \delta(z-w)$, e se $n \leq j$ e caso $n>j$, então:

$$
\begin{aligned}
(z-w)^{n} \partial_{w}^{(j)} \delta(z-w) & =(z-w)^{n-j-1}(z-w)^{j+1} \partial_{w}^{(j)} \delta(z-w) \\
& =(z-w)^{n-j-1} \cdot 0 \\
& =0
\end{aligned}
$$

(2) Tem-se que:

$\partial_{w}^{(j-n)} \delta(z-w)=\sum_{m \in \mathbb{Z}}\left(\begin{array}{c}m \\ j-n\end{array}\right) z^{-m-1} w^{m-j+n}$

logo o coeficiente em $z^{-1}$ é obtido quando $m=0$.

Para auxiliar o estudo das distribuições formais do tipo (1.11), introduzse as seguintes definições:

Definição 1.7. Defina $U\left[\left[z, z^{-1}, w, w^{-1}\right]\right]^{\circ} \quad$ como o subespaço de $U\left[\left[z, z^{-1}, w, w^{-1}\right]\right]$ que consiste das distribuições formais $U$-valuadas $a(z, w)$ tais que:

$$
\pi a(z, w)=\sum_{j=0}^{\infty}\left(\operatorname{Res}_{z} a(z, w) \cdot(z-w)^{j}\right) \cdot \partial_{w}^{(j)} \delta(z-w)
$$


Definição 1.8. Dada $a(z, w) \in U\left[\left[z, z^{-1}, w, w^{-1}\right]\right]$, defina:

$$
a(z, w)^{+(z)}=\sum_{\substack{m \in \mathbb{Z}_{+} \\ n \in \mathbb{Z}}} a_{m, n} z^{m} w^{n}
$$

Diz-se que uma distribuição formal a $(z, w)$ é holomorfa em $z$ se:

$$
a(z, w)=a(z, w)^{+(z)}
$$

Desta forma, tem-se a seguinte proposição:

Proposição 1.9. São verdadeiras as seguintes afirmações:

(i) A aplicação $\pi$ é uma projeção em $U\left[\left[z, z^{-1}, w, w^{-1}\right]\right]^{\circ}$.

(ii) $\operatorname{Ker\pi }=\left\{a(z, w) \in U\left[\left[z, z^{-1}, w, w^{-1}\right]\right]^{\circ}\right.$ que são holomorfas em $\left.z\right\}$.

(iii) Qualquer distribuição formal $a(z, w)$ de $U\left[\left[z, z^{-1}, w, w^{-1}\right]\right]^{\circ} \quad e ́$ univocamente representada na forma:

$$
a(z, w)=\sum_{j=0}^{\infty} c^{j}(w) \partial_{w}^{(j)} \delta(z-w)+b(z, w)
$$

onde $b(z, w)$ é uma distribuição formal holomorfa em z, e os coeficientes $c^{j}(w)$ são dados por (1.12).

Demonstração. Tem-se que $a(z, w)=\sum_{\substack{m \in \mathbb{Z}_{+} \\ n \in \mathbb{Z}}} a_{m, n} \cdot z^{m} \cdot w^{n}$. Portanto:

$$
\begin{aligned}
\pi a(z, w) & =\sum_{j=0}^{\infty}\left(\operatorname{Res}_{z} a(z, w) \cdot(z-w)^{j}\right) \cdot \partial_{w}^{(j)} \delta(z-w) \\
& =\sum_{j=0}^{\infty} c^{j}(w) \cdot \partial_{w}^{(j)} \delta(z-w)
\end{aligned}
$$

$$
\begin{aligned}
\pi^{2} a(z, w) & =\sum_{j=0}^{\infty}\left(\operatorname{Res}_{z} \pi a(z, w) \cdot(z-w)^{j}\right) \cdot \partial_{w}^{(j)} \delta(z-w) \\
& =\sum_{j=0}^{\infty}\left(\operatorname{Res}_{z} \sum_{i=0}^{\infty} c^{i}(w) \cdot \partial_{w}^{(i)} \delta(z-w) \cdot(z-w)^{j}\right) \partial_{w}^{(j)} \delta(z-w)
\end{aligned}
$$

Assim, para mostrar que $\pi^{2} a(z, w)=\pi a(z, w)$, basta mostrar que: 
$\operatorname{Res}_{z} \sum_{i=0}^{\infty} c^{i}(w) \cdot \partial_{w}^{(i)} \delta(z-w) \cdot(z-w)^{j}=c^{j}(w)$

De fato:

$$
\begin{aligned}
& \operatorname{Res}_{z} \sum_{i=0}^{\infty} c^{i}(w) \cdot \partial_{w}^{(i)} \delta(z-w)(z-w)^{j} \\
& =\operatorname{Res}_{z} \sum_{i=j}^{\infty} c^{i}(w) \cdot \partial_{w}^{(i-j)} \delta(z-w) \\
& =\operatorname{Res}_{z} \sum_{i=0}^{\infty} c^{i+j}(w) \cdot \partial_{w}^{(i)} \delta(z-w) \\
& =\operatorname{Res}_{z} \sum_{i=0}^{\infty} c^{i+j}(w) \cdot \sum_{m=0}^{\infty}\left(\begin{array}{c}
m \\
i
\end{array}\right) z^{-m-1} w^{m-i} \\
& =\sum_{i=0}^{\infty} c^{i+j}(w) \cdot\left(\begin{array}{c}
0 \\
i
\end{array}\right) w^{-i} \\
& =c^{j}(w)
\end{aligned}
$$

Logo, $\pi$ é uma projeção em $U\left[\left[z, z^{-1}, w, w^{-1}\right]\right]^{\circ}$, o que prova $(i)$.

Agora se $a(z, w)$ é holomorfa em $z$, então

$$
a(z, w)=a(z, w)^{+(z)}=\sum_{\substack{m \in \mathbb{Z}_{+} \\ n \in \mathbb{Z}}} a_{m, n} z^{m} w^{n}
$$

Assim, dizer que $a(z, w)$ é holomorfa em $z$ equivale a dizer que $a(z, w)$ possui apenas potências não-negativas de $z$, e como $(z-w)^{n}$, para $n \geq 0$, também possui apenas potências não-negativas de $z$, segue que o seu produto também. Logo:

$$
\begin{aligned}
\pi a(z, w) & =\sum_{j=0}^{\infty}\left(\operatorname{Res}_{z} a(z, w) \cdot(z-w)^{j}\right) \cdot \partial_{w}^{(j)} \delta(z-w) \\
& =\sum_{j=0}^{\infty} 0 \cdot \partial_{w}^{(j)} \delta(z-w) \\
& =0
\end{aligned}
$$


Por outro lado, se $a(z, w) \in K e r \pi$, então:

$$
\begin{aligned}
0 & =\pi a(z, w) \\
& =\sum_{j=0}^{\infty} c^{j}(w) \cdot \partial_{w}^{(j)} \delta(z-w) \\
& =\sum_{j=0}^{\infty} c^{j}(w) \sum_{m \in \mathbb{Z}}\left(\begin{array}{c}
m \\
j
\end{array}\right) z^{-m-1} w^{m-j} \\
& =\sum_{j \in \mathbb{Z}_{+}} \sum_{m \in \mathbb{Z}} c^{j}(w)\left(\begin{array}{c}
m \\
j
\end{array}\right) z^{-m-1} w^{m-j}
\end{aligned}
$$

Desta forma, tem-se que:

$$
\left(\begin{array}{c}
m \\
j
\end{array}\right) c^{j}(w)=0, \forall m \in \mathbb{Z}, j \in \mathbb{Z}_{+}
$$

e $\operatorname{assim}, c^{j}(w)=0, \forall j \in \mathbb{Z}_{+}$.

Agora $c^{0}(w)=0 \Rightarrow \operatorname{Res}_{z} a(z, w)=0 \Rightarrow a_{-1, n}=0, \forall n \in \mathbb{Z}$. Suponhamos o resultado válido para $j<k$ e provemos para $j=k$.

$$
\begin{aligned}
c^{k}(w)=0 & \Rightarrow \operatorname{Res}_{z} a(z, w) \cdot(z-w)^{k} \\
& =\operatorname{Res}_{z}\left[a(z, w) z^{k}+a(z, w) \sum_{j=0}^{k-1}\left(\begin{array}{l}
k \\
j
\end{array}\right) z^{j} w^{k-j}=0\right. \\
& \Rightarrow a_{-k, n}+\sum_{j=0}^{k-1}\left(\begin{array}{l}
k \\
j
\end{array}\right) a_{-j, n+k-j}=0 \\
& \Rightarrow a_{-k, n}+\sum_{j=0}^{k-1}\left(\begin{array}{l}
k \\
j
\end{array}\right) 0=0
\end{aligned}
$$

Logo $a_{-k, n}=0, \forall n \in \mathbb{N}$, o que prova $(i i)$. Finalmente, provemos (iii).

$$
\begin{aligned}
\text { Como } \pi a(z, w)=\sum_{j=0}^{\infty} c^{j}(w) \partial_{w}^{(j)} \delta(z-w) & \in \operatorname{Im} \pi, \text { temos que: } \\
b(z, w)=a(z, w)-\pi a(z, w) \Rightarrow \pi b(z, w) & =\pi a(z, w)-\pi^{2} a(z, w) \\
& =\pi a(z, w)-\pi a(z, w) \\
& =0
\end{aligned}
$$


$\operatorname{Logo} b(z, w) \in \operatorname{Ker} \pi$ e assim, $U\left[\left[z, z^{-1}, w, w^{-1}\right]\right]^{\circ}=\operatorname{Im} \pi+\operatorname{Ker} \pi$.

Nos falta apenas mostrar que a soma é direta. De fato:

$a(z, w) \in \operatorname{Ker} \pi \bigcap \operatorname{Im} \pi \Rightarrow \exists a_{1}(z, w)$ tal que $a(z, w)=\pi a_{1}(z, w)$.

Agora $a(z, w) \in K e r \pi$. Então:

$$
\begin{aligned}
0 & =\pi a(z, w) \\
& =\pi^{2} a_{1}(z, w) \\
& =\pi a_{1}(z, w)=a(z, w)
\end{aligned}
$$

Logo $\operatorname{Im} \pi \bigcap \operatorname{Ker} \pi=0$ e assim:

$$
U\left[\left[z, z^{-1}, w, w^{-1}\right]\right]=\operatorname{Im} \pi \oplus \operatorname{Ker} \pi
$$

Corolário 1.10. O subespaço de $U\left[\left[z, z^{-1}, w, w^{-1}\right]\right]$ dos operadores que são anulados por $(z-w)^{N}$, com $N \geqslant 1$ fixado, é da forma:

$$
\sum_{j=0}^{N-1} \partial^{(j)} \delta(z-w) U\left[w, w^{-1}\right]
$$

onde todo elemento a $(z, w)$ de (1.15) é univocamente representado na forma:

$$
a(z, w)=\sum_{j=0}^{N-1} c^{j}(w) \partial^{(j)} \delta(z-w)
$$

com $c^{j}(w)$ sendo dado pelo Teorema 1.6.

Demonstração. Por um lado, temos:

$$
\begin{aligned}
& (z-w)^{N} \cdot \sum_{j=0}^{N-1} c^{j}(w) \cdot \partial_{w}^{(j)} \delta(z-w) \\
& =\sum_{j=0}^{N-1}(z-w)^{N-j-1} \cdot c^{j}(w) \cdot(z-w)^{j+1} \cdot \partial_{w}^{(j)} \delta(z-w) \\
& =\sum_{j=0}^{N-1}(z-w)^{N-j-1} \cdot c^{j}(w) \cdot 0 \\
& =0
\end{aligned}
$$


Por outro lado, temos que $(z-w)^{N} \cdot a(z, w)=0$, e então $a(z, w) \in$ $U\left[\left[z, z^{-1}, w, w^{-1}\right]\right]^{\circ}$, pois:

$$
\begin{aligned}
\pi a(z, w) & =\sum_{j=0}^{\infty}\left(\left(\operatorname{Res}_{z} a(z, w) \cdot(z-w)^{j}\right) \cdot \partial_{w}^{(j)} \delta(z-w)\right) \\
& =\sum_{j=0}^{N-1}\left(\operatorname{Res}_{z} a(z, w) \cdot(z-w)^{j}\right) \cdot \partial_{w}^{(j)} \delta(z-w) \\
& +\sum_{j=N}^{\infty}\left(\operatorname{Res}_{z} 0\right) \cdot \partial_{w}^{(j)} \delta(z-w) \\
& =\sum_{j=0}^{N-1}\left(\operatorname{Res}_{z} a(z, w) \cdot(z-w)^{j}\right) \cdot \partial_{w}^{(j)} \delta(z-w)
\end{aligned}
$$

logo converge pois temos uma soma finita de termos que converge.

Assim, segue de (1.14) e do Teorema 1.5 (vi) que:

$$
\begin{aligned}
0 & =(z-w)^{N} \cdot a(z, w) \\
& =\sum_{j=0}^{\infty} c^{j}(w) \cdot(z-w)^{N} \cdot \partial_{w}^{(j)} \delta(z-w)+(z-w)^{N} \cdot b(z, w) \\
& =\sum_{j=N}^{\infty} c^{j}(w) \cdot \partial_{w}^{(j-N)} \delta(z-w)+(z-w)^{N} \cdot b(z, w)
\end{aligned}
$$

Da unicidade da Proposição $1.9(i i i)$, temos que $c^{j}(w)=0$ para $j \geqslant N$ e $(z-w)^{N} b(z, w)=0, \operatorname{logo} b(z, w)=0$, já que $b(z, w)=\sum_{n \in \mathbb{Z}_{+}} a_{n}(w) z^{n}$.

Definição 1.11. Uma distribuição formal $a(z, w)$ é dita local se

$$
(z-w)^{N} \cdot a(z, w)=0
$$

$\operatorname{para} N \gg 0$.

O Corolário acima nos diz que qualquer distribuição formal local $a(z, w)$ tem expansão (1.16), chamada expansão $O P E$ de $a(z, w)$. Os $c^{n}(w)$ de (1.16) são chamados coeficientes $O P E$ de $a(z, w)$. Maiores detalhes sobre os coeficientes $O P E$ serão dados no decorrer deste trabalho. 
Para simplificar a notação, ao invés de escrever

$$
a(z, w)=\sum_{\substack{m \in \mathbb{Z} \\ n \in \mathbb{Z}}} a_{m, n} z^{m} w^{n}
$$

podemos escrever

$$
a(z, w)=\sum_{\substack{m \in \mathbb{Z} \\ n \in \mathbb{Z}}} a_{(m, n)} z^{-m-1} w^{-n-1}
$$

Desta forma, teremos $a_{(n)}=\operatorname{Res}_{z} a(z) z^{n}$ (evitando $a_{n}=\operatorname{Res}_{z} a(z) z^{-n-1}$ )

Teorema 1.12. A expansão (1.16) é equivalente a:

$$
a_{(m, n)}=\sum_{j=0}^{N-1}\left(\begin{array}{c}
m \\
j
\end{array}\right) c_{(n+m-j)}^{j}
$$

Demonstração. De fato, temos que:

$$
\begin{aligned}
a_{(m, n)} & =\operatorname{Res}_{z} \operatorname{Res}_{w} a(z, w) z^{m} w^{n} \\
& =\operatorname{Res}_{z} \operatorname{Res}_{w} \sum_{j=0}^{N-1} c^{j}(w) \partial_{w}^{(j)} \delta(z-w) z^{m} w^{n} \\
& =\operatorname{Res}_{z} \operatorname{Res}_{w} \sum_{j=0}^{N-1} c^{j}(w) \sum_{i \in \mathbb{Z}}\left(\begin{array}{l}
i \\
j
\end{array}\right) z^{-i-1} w^{i-j} z^{m} w^{n} \\
& =\operatorname{Res}_{z} \operatorname{Res}_{w} \sum_{j=0}^{N-1} \sum_{i \in \mathbb{Z}} c^{j}(w)\left(\begin{array}{l}
i \\
j
\end{array}\right) z^{m-i-1} w^{n+i-j} \\
& =\operatorname{Res}_{w} \sum_{j=0}^{N-1} c^{j}(w)\left(\begin{array}{c}
m \\
j
\end{array}\right) w^{n+m-j} \\
& =\operatorname{Res}_{w} \sum_{j=0}^{N-1}\left(\begin{array}{c}
m \\
j
\end{array}\right) \sum_{i \in \mathbb{Z}} c_{(i)}^{j} w^{-i-1} w^{n+m-j} \\
& =\sum_{j=0}^{N-1}\left(\begin{array}{c}
m \\
j
\end{array}\right) c_{(n+m-j)}^{j}
\end{aligned}
$$

A outra aplicação é obtida de forma análoga. 


\subsection{A Transformada Formal de Fourier}

Com o intuito de facilitar o cálculo dos coeficientes $O P E$ no Capítulo 2 e para melhor trabalhar com a teoria de superálgebra conforme no Capítulo 3 , será utilizada a definição abaixo:

Definição 1.13. Chama-se de Transformada Formal de Fourier de uma distribuição formal $a(z, w)$ a aplicação $\mathbb{C}$-linear de $U\left[\left[z, z^{-1}, w, w^{-1}\right]\right]^{\circ} \mathrm{em}$ $U\left[\left[w, w^{-1}\right]\right][[\lambda]]$ dada por:

$$
F_{z, w}^{\lambda}(a(z, w))=\operatorname{Res}_{z} e^{\lambda(z-w)} a(z, w)
$$

Teorema 1.14. São válidas as seguintes relações:

(i) $F_{z, w}^{\lambda}\left(\partial_{w}^{j} \delta(z-w)\right)=\lambda^{j}$

(ii) $F_{z, w}^{\lambda}(a(z, w))=\sum_{n \in \mathbb{Z}_{+}} \lambda^{(n)} c^{n}(w)$

onde $c^{n}(w)$ é dado pelo teorema 1.6 e $\lambda^{(n)}=\frac{\lambda^{n}}{n !}$.

(iii) $e^{\lambda(z-w)} \partial_{w}^{j} \delta(z-w)=\left(\lambda+\partial_{w}\right)^{j} \delta(z-w)$

(iv) $F_{z, w}^{\lambda} \partial_{z}=-\lambda F_{z, w}^{\lambda}=\left[\partial_{w}, F_{z, w}^{\lambda}\right]$

(v) $F_{z, w}^{\lambda} a(w, z)=F_{z, w}^{-\lambda-\partial_{w}} a(z, w)$, se $a(z, w)$ for local.

Demonstração. Temos: 


$$
\begin{aligned}
F_{z, w}^{\lambda}\left(\partial_{w}^{j} \delta(z-w)\right) & =\operatorname{Res}_{z} e^{\lambda(z-w)} \cdot \partial_{w}^{j} \delta(z-w) \\
& =\operatorname{Res}_{z} \sum_{n \in \mathbb{N}} \frac{1}{n !} \cdot \lambda^{n} \cdot(z-w)^{n} \cdot j ! \cdot \partial_{w}^{(j)} \delta(z-w) \\
& =\operatorname{Res}_{z} \sum_{n=0}^{j} \frac{j !}{n !} \lambda^{n} \partial_{w}^{(j-n)} \delta(z-w) \\
& =\operatorname{Res}_{z} \sum_{n=0}^{j} \frac{j !}{n !} \lambda^{n} \sum_{m \in \mathbb{Z}}\left(\begin{array}{c}
m \\
j-n
\end{array}\right) z^{-m-1} w^{m-j+n} \\
& =\sum_{n=0}^{j} \frac{j !}{n !} \lambda^{n}\left(\begin{array}{c}
0 \\
j-n
\end{array}\right) w^{n-j} \\
& =\frac{j !}{j !} \lambda^{j}\left(\begin{array}{c}
0 \\
0
\end{array}\right) w^{0} \\
& =\lambda^{j}
\end{aligned}
$$

o que prova (i). Agora temos:

$$
\begin{aligned}
F_{z, w}^{\lambda}(a(z, w)) & =\operatorname{Res}_{z} e^{\lambda(z-w)}\left(\sum_{m \in \mathbb{Z}_{+}} c^{j}(w) \partial_{w}^{(j)} \delta(z-w)+b(z, w)\right) \\
& =\operatorname{Res}_{z}\left(\sum_{m \in \mathbb{Z}_{+}} c^{j}(w) e^{\lambda(z-w)} \frac{1}{j !} \partial_{w}^{j} \delta(z-w)\right)+\operatorname{Res}_{z} e^{\lambda(z-w)} b(z, w) \\
& =\sum_{m \in \mathbb{Z}_{+}} \frac{c^{j}(w)}{j !} \operatorname{Res}_{z} e^{\lambda(z-w)} \partial_{w}^{j} \delta(z-w)+\operatorname{Res}_{z} e^{\lambda(z-w)} b(z, w) \\
& \stackrel{(\star)}{=} \sum_{m \in \mathbb{Z}_{+}} \frac{c^{n}(w)}{n !} \lambda^{n}+0 \\
& =\sum_{m \in \mathbb{Z}_{+}} c^{n}(w) \lambda^{(n)}
\end{aligned}
$$

onde vale $(\star)$ já que $e^{\lambda(z-w)}=\sum_{n \in \mathbb{N}} \frac{1}{n !} \lambda^{n}(z-w)^{n}$ só possui potências nãonegativas de $\mathrm{z}$, assim como $b(z, w)$. Isto prova (ii). Agora provemos (iii) e $(i v)$ : 


$$
\begin{aligned}
e^{\lambda(z-w)} \partial_{w}^{j} \delta(z-w) & =\sum_{n=0}^{\infty} \frac{j !}{n !} \lambda^{n}(z-w)^{n} \partial_{w}^{(j)} \delta(z-w) \\
& =\sum_{n=0}^{j} \frac{j !}{n !} \lambda^{n} \partial_{w}^{(j-n)} \delta(z-w) \\
& =\sum_{n=0}^{j} \frac{j !}{n !(j-n) !} \lambda^{n} \partial_{w}^{j-n} \delta(z-w) \\
= & \sum_{n=0}^{j}\left(\begin{array}{l}
j \\
n
\end{array}\right) \lambda^{n} \partial_{w}^{j-n} \delta(z-w) \\
& =\left(\lambda+\partial_{w}\right)^{j} \delta(z-w) \\
F_{z, w}^{\lambda} \partial_{z}(a(z, w))= & \operatorname{Res}_{z} e^{\lambda(z-w)} \partial_{z} a(z, w) \\
= & -\operatorname{Res}_{z} \partial_{z}\left(\sum_{n \in \mathbb{N}} \frac{1}{n !} \lambda^{n}(z-w)^{n}\right) \cdot a(z, w) \\
= & \left.-\operatorname{Res}_{z} \sum_{n \in \mathbb{N}} \frac{1}{n !} \lambda^{n} \cdot n(z-w)^{n-1}\right) \cdot a(z, w) \\
= & 0-\operatorname{Res}_{z} \sum_{n \in \mathbb{N}^{*}} \frac{1}{(n-1) !} \lambda \cdot \lambda^{n-1}(z-w)^{n-1} \cdot a(z, w) \\
= & -\operatorname{Res}_{z} \sum_{n \in \mathbb{N}} \frac{1}{n !} \lambda \cdot \lambda^{n}(z-w)^{n} \cdot a(z, w) \\
= & \lambda \cdot F_{z, w}^{\lambda}(a(z, w))
\end{aligned}
$$

Agora:

$$
\begin{aligned}
{\left[\partial_{w}, F_{z, w}^{\lambda}\right] a(z, w) } & =\partial_{w} F_{z, w}^{\lambda} a(z, w)-F_{z, w}^{\lambda} \partial_{w} a(z, w) \\
& =\partial_{w}\left(\operatorname{Res}_{z} e^{\lambda(z-w)} a(z, w)\right)-\operatorname{Res}_{z} e^{\lambda(z-w)} \partial_{w} a(z, w) \\
& =\operatorname{Res}_{z}\left[\partial_{w}\left(e^{\lambda(z-w)} a(z, w)\right)-e^{\lambda(z-w)} \partial_{w} a(z, w)\right] \\
& -\operatorname{Res}_{z} e^{\lambda(z-w)} \partial_{w} a(z, w) \\
& =-\lambda \operatorname{Res}_{z} e^{\lambda(z-w)} a(z, w) \\
& =-\lambda F_{z, w}^{\lambda}(a(z, w))
\end{aligned}
$$

Finalmente, provemos $(v)$ : 


$$
\begin{aligned}
F_{z, w}^{\lambda}(a(w, z)) & =\operatorname{Res}_{z}\left(e^{\lambda(z-w)} c(z) \partial_{z}^{k} \delta(z-w)\right) \\
& =(-1)^{k} \operatorname{Res}_{z} c(z) e^{\lambda(z-w)} \partial_{w}^{k} \delta(z-w) \\
& =(-1)^{k} \operatorname{Res}_{z} c(z) \cdot\left(\lambda+\partial_{w}\right)^{k} \cdot \delta(z-w) \\
& =\left(-\lambda-\partial_{w}\right)^{k} c(w) \\
& =\operatorname{Res}_{z} c(w) \cdot\left(-\lambda-\partial_{w}\right)^{k} \cdot \delta(z-w) \\
& =\operatorname{Res}_{z}\left(c(w) \cdot e^{\left(-\lambda-\partial_{w}\right) \cdot(z-w)} \partial_{w}^{k} \delta(z-w)\right) \\
& =F_{z, w}^{-\lambda-\partial_{w}} a(z, w)
\end{aligned}
$$

Teorema 1.15. As fórmulas do Teorema anterior, itens $(i v)$ e $(v)$ são equivalentes às seguintes relações dos coeficientes OPE:

(i) $c_{z}^{n}(w)=-n \cdot c^{n-1}(w)$

(ii) $c_{w}^{n}(w)=\partial_{w} c^{n}(w)+n \cdot c^{n-1}(w)$

(iii) $\tilde{c}^{n}(w)=\sum_{j \in \mathbb{Z}_{+}}(-1)^{n+j} \partial_{w}^{(j)} c^{n+j}(w)$

onde $c_{z}^{n}(w), c_{w}^{n}(w), \tilde{c}^{n}(w)$ são os coeficientes OPE de $\partial_{z} a(z, w), \partial_{w} a(z, w)$, e de $\tilde{a}(z, w)$, onde $\tilde{a}(z, w)=a(w, z)$.

Demonstração. Temos que:

$$
\begin{aligned}
c_{z}^{n}(w) & =\operatorname{Res}_{z} \partial_{z} a(z, w) \cdot(z-w)^{n} \\
& =\operatorname{Res}_{z} a(z, w) \cdot \partial_{z}(z-w)^{n} \\
& =-n \cdot \operatorname{Res}_{z} a(z, w) \cdot(z-w)^{n-1} \\
& =-n \cdot c^{n-1}(w)
\end{aligned}
$$

e também que

$$
\begin{aligned}
c_{w}^{n}(w) & =\operatorname{Res}_{z} \partial_{w} a(z, w) \cdot(z-w)^{n} \\
& =-\operatorname{Res}_{z}\left[\partial_{w}\left(a(z, w) \cdot(z-w)^{n}\right)-a(z, w) \cdot \partial_{w}(z-w)^{n}\right] \\
& =\partial_{w}\left(\operatorname{Res}_{z} a(z, w) \cdot(z-w)^{n}\right)+n \cdot \operatorname{Res}_{z} a(z, w) \cdot(z-w)^{n-1} \\
& =\partial_{w} c^{n}(w)+n \cdot c^{n-1}(w)
\end{aligned}
$$

o que prova $(i)$ e $(i i)$. Agora: 


$$
\begin{aligned}
\tilde{c}^{n}(w) & =\operatorname{Res}_{z} a(w, z) \cdot(z-w)^{n} \\
& =\operatorname{Res}_{z} \sum_{j=0}^{\infty} c^{j}(z) \cdot \partial_{z}^{(j)} \delta(z-w)(z-w)^{n} \\
& =\operatorname{Res}_{z} \sum_{j=n}^{\infty}(-1)^{j} \cdot c^{j}(z) \cdot \partial_{z}^{(j-n)} \delta(z-w) \\
& =\sum_{j=n}^{\infty}(-1)^{n} \cdot(-1)^{j-n} \cdot \operatorname{Res}_{z} \partial_{z}^{(j-n)} c^{j}(z) \cdot \delta(z-w) \\
& =\sum_{j=n}^{\infty}(-1)^{j} \partial_{w}^{(j-n)} c^{j}(w) \\
& =\sum_{j \in \mathbb{Z}_{+}}(-1)^{n+j} \partial_{w}^{(j)} c^{n+j}(w)
\end{aligned}
$$

o que prova $(i i i)$.

Uma composição de duas transformadas de Fourier $F_{z, w}^{\lambda} F_{x, w}^{\mu}$ é uma aplicação $\mathbb{C}$-linear de $U\left[\left[z, z^{-1}, w, w^{-1}, x, x^{-1}\right]\right]$ em $U\left[\left[w, w^{-1}\right]\right][[\lambda, \mu]]$. O resultado abaixo ajuda bastante na hora de fazer os cálculos:

Teorema 1.16. Vale a igualdade:

$F_{z, w}^{\lambda} F_{x, w}^{\mu}=F_{x, w}^{\lambda+\mu} F_{z, w}^{\lambda}$

Demonstração. De fato, temos:

$$
\begin{aligned}
F_{z, w}^{\lambda} F_{x, w}^{\mu} a(z, w, x) & =\operatorname{Re}_{z} e^{\lambda(z-w)} \operatorname{Res}_{x} e^{\mu(x-w)} a(z, w, x) \\
& =\operatorname{Res}_{z} \operatorname{Res}_{x} e^{\lambda z-\lambda w+\mu x-\mu w} a(z, w, x) \\
& =\operatorname{Res}_{x} \operatorname{Res}_{z} e^{(\lambda x-\lambda \mu+\mu x-\mu w)+(\lambda z-\lambda w)} a(z, w, x) \\
& =\operatorname{Res}_{x} \operatorname{Res}_{z} e^{(\lambda+\mu)(x-w)} e^{\lambda(z-w)} a(z, w, x) \\
& =F_{x, w}^{\lambda+\mu} F_{z, w}^{\lambda} a(z, w, x)
\end{aligned}
$$

Da arbitrariedade de $a(z, w, x)$, segue o resultado.

Uma linguagem alternativa a das distribuições formais locais U-valuadas em $z$ e $w$ é a linguagem dos operadores diferenciáveis de $U\left[w, w^{-1}\right]$ em $U\left[\left[w, w^{-1}\right]\right]$. Então, para uma distribuição formal $a(z, w)$, o operador associado será: 


$$
\left(D_{a(z, w)} f\right)(w)=\operatorname{Res}_{z}(a(z, w) \cdot f(z))
$$

Desta forma, temos:

Teorema 1.17. Valem as seguintes igualdades:

(i) $D_{\partial_{w}^{k} \delta(z-w)}=\partial_{w}^{k}, \forall k \in \mathbb{Z}_{+}$;

(ii) Se $a(z, w)=\sum_{k \in \mathbb{Z}} c^{k}(w) \partial_{w}^{(k)} \delta(z-w)$, então $D_{a(z, w)}=\sum_{k \in \mathbb{Z}} c^{k}(w) \partial_{w}^{(k)}$;

(iii) $D_{a(w, z)}=\sum_{k \in \mathbb{Z}}\left(-\partial_{w}\right)^{(k)} c^{k}(w)$.

Demonstração. Para provar $(i)$, é suficiente provar para o caso $f(z)=z^{i}$, com $i \in \mathbb{Z}$ qualquer, já que o mesmo seguirá por linearidade. Sendo assim,

$$
\begin{aligned}
\left(D_{\partial_{w}^{k} \delta(z-w)} f\right)(w) & =\operatorname{Res}_{z} \partial_{w}^{k} \delta(z-w) f(z) \\
& =\operatorname{Res}_{z} \sum_{m \in \mathbb{Z}}\left(\begin{array}{c}
m \\
k
\end{array}\right) k ! z^{-m-1} w^{m-k} z^{i} \\
& =\left(\begin{array}{c}
i \\
k
\end{array}\right) k ! w^{i-k} \\
& =\frac{i !}{(i-k) !} w^{i-k} \\
& =\partial_{w}^{k} w^{i} \\
& =\partial_{w}^{k} f(w)
\end{aligned}
$$

o que prova $(i)$. Agora provemos $(i i)$ e $(i i i)$ :

$$
\begin{aligned}
\left(D_{a(z, w)} f\right)(w) & =\operatorname{Res}_{z} a(z, w) f(z) \\
& =\operatorname{Res}_{z} \sum_{k \in \mathbb{Z}} c^{k}(w) \partial_{w}^{(k)} \delta(z-w) f(z) \\
& =\sum_{k \in \mathbb{Z}} c^{k}(w)\left[\operatorname{Res}_{z} \frac{1}{k !} \partial_{w}^{k} \delta(z-w) f(z)\right] \\
& \stackrel{(1)}{=} \sum_{k \in \mathbb{Z}} c^{k}(w) \frac{\partial_{w}^{k}}{k !} f(w) \\
& =\sum_{k \in \mathbb{Z}} c^{k}(w) \partial_{w}^{(k)} f(w)
\end{aligned}
$$


Analogamente, $\left(D_{a(w, z)} f\right)(w)=\sum_{k \in \mathbb{Z}}\left(-\partial_{w}\right)^{(k)} c^{k}(w) f(w)$ (veja Teorema $1.14(v))$ 


\section{Capítulo 2}

\section{Superálgebras de Lie de distribuições formais}

\subsection{Introdução}

Neste Capítulo, introduz-se o conceito de superálgebras de Lie de distribuições formais e define-se o conceito de localidade de duas distribuições formais e suas equivalências através do Teorema 2.8. Também será definido, analogamente ao feito no Capítulo 1 , o conceito de $O P E$, o qual será utilizado no decorrer do trabalho.

Na Seção 2.3, faz-se uso da transformada formal de Fourier vista no final do Capítulo 1 para definir os conceitos de $\lambda$-produto e de $\lambda$-colchete, facilitando bastante o estudo dos coeficientes $O P E$. Já na Seção 2.5, exemplos de superálgebras de Lie são apresentados dando uma atenção especial para as álgebras de Virasoro e para as álgebras de Correntes, já que estas duas aparecem nos resultados finais do Capítulo 3, e também do Capítulo 4. Quanto à Seção 2.6, são apresentados exemplos de superálgebras de Lie de distribuições formais, que serão de grande valia em trabalhos futuros. Para finalizar, os conceitos e resultados principais de peso conforme são abordados na Seção 2.7 .

Dessa forma, tem-se a seguinte definição:

Definição 2.1. Seja $\mathbb{Z}_{2}=\{\overline{0}, \overline{1}\}$. Um espaço vetorial $U$ da forma $U=$ $U_{\overline{0}} \oplus U_{\overline{1}}$, onde $U_{\overline{0}}$ e $U_{\overline{1}}$ são subespaços vetoriais, é chamado de espaço vetorial $\mathbb{Z}_{2 \text {-graduado. }}$

Os subespaços $U_{\overline{0}}$ e $U_{\overline{1}}$ são chamados respectivamente de parte par e parte ímpar de $U$. Se $u \in U$ é tal que $u \in U_{\bar{i}}$, com $u$ não-nulo, então $u$ é dito homogêneo e, assim, definimos a paridade de $u$ (e a denotamos por 
$p(u))$ como sendo igual à $i$. Desta forma, para qualquer elemento homogêneo não-nulo $u \in U$, o inteiro $(-1)^{p(u)}$ estará bem definido (o termo "não-nulo" é usado apenas para enfatizar).

Definição 2.2. Uma álgebra de Lie consiste de um espaço vetorial $\mathfrak{g}$ sobre um corpo $\mathbb{K}$ (no nosso caso $\mathbb{C}$ ) munido de um operador bilinear

$$
[\cdot, \cdot] \mathfrak{g} \times \mathfrak{g} \longrightarrow \mathfrak{g}
$$

que satisfaça, $\forall x, y, z \in \mathfrak{g}:$

(a) $[x, y]=-[y, x]$ (anti-simetria)

(b) $[x,[y, z]]+[y,[z, x]]+[z,[x, y]]=0$ (identidade de Jacobi)

O operador $[\cdot, \cdot]$ é chamado de colchete de Lie.

Uma álgebra de Lie com colchete de Lie trivial é chamada comutativa. A estrutura de uma álgebra de Lie pode ser vista em muitos lugares. Por exemplo, toda algebra associativa $A$ pode ser considerada como uma álgebra de Lie definindo $[x, y]=x y-y x, \forall x, y \in A$. Uma expressão desta forma é chamada alternador.

Definição 2.3. Um espaço vetorial $\mathbb{Z}_{2}$-graduado $\mathfrak{g}=\mathfrak{g}_{0} \oplus \mathfrak{g}_{\overline{1}}$ sobre $\mathbb{K}$ munido de um operador bilinear

$$
[\cdot, \cdot] \mathfrak{g} \times \mathfrak{g} \longrightarrow \mathfrak{g}
$$

é chamado de superálgebra de Lie se valer o seguinte:

(a) $\left[\mathfrak{g}_{i}, \mathfrak{g}_{j}\right] \subset \mathfrak{g}_{i+j}, \forall i, j \in \mathbb{Z}_{2}$

(b) $[x, y]=-(-1)^{p(x) p(y)}[y, x]$ (anti-supersimetria)

(c) $[x,[y, z]]=(-1)^{p(x) p(y)}[y,[x, z]]+[[x, y], z]$ (superidentidade de Jacobi)

onde para as duas últimas condições, $x, y, z$ são elementos homogêneos de $\mathfrak{g}$.

O operador $[\cdot, \cdot \cdot]$ é chamado de supercolchete de Lie. Claramente, a subálgebra $\mathfrak{g}_{0}$ é uma álgebra de Lie. 


\subsection{O conceito de localidade de duas distribuições formais numa superálgebra de Lie}

Definição 2.4. Duas distribuições formais a $(z)$ e $b(z)$ com valores numa superálgebra de Lie $\mathfrak{g}$ são chamadas mutuamente locais (ou locais, ou formam um par local) se a distribuição formal $[a(z), b(w)] \in \mathfrak{g}\left[\left[z, z^{-1}, w, w^{-1}\right]\right]$ é local, isto é,

$$
(z-w)^{N}[a(z), b(w)]=0
$$

para $N \gg 0$.

Teorema 2.5. Se $a(z)$ e b(z) são locais, então $\partial a(z)$ e b(z) também o são.

Demonstração. De fato,

$$
\begin{aligned}
& (z-w)^{N+1}[a(z), b(w)]=0 \Rightarrow \partial\left((z-w)^{N+1}[a(z), b(w)]\right)=0 \\
& \Rightarrow(N+1)(z-w)^{N}\left[a(z),(b(w)]+(z-w)^{N+1}[\partial a(z), b(w)]=0\right. \\
& \Rightarrow(z-w)^{N+1}[\partial a(z), b(w)]=0
\end{aligned}
$$

Definição 2.6. Dada uma distribuição formal $a(z)=\sum_{n \in \mathbb{Z}} a_{(n)} z^{-n-1} e$ $b(z)=\sum_{m \in \mathbb{Z}} b_{(m)} z^{-m-1}$, define-se:

(a) $a(z)_{-}=\sum_{n \geq 0} a_{(n)} z^{-n-1}$

(b) $a(z)_{+}=\sum_{n<0} a_{(n)} z^{-n-1}$

(c) $: a(z) b(w):=a(z)_{+} b(w)+(-1)^{p(a) p(b)} b(w) a(z)_{-}$

onde $p(a)=i \in \mathbb{Z}_{2}$ é definido quando a(z) é homogêneo (isto é, quando $\left.p\left(a_{(n)}\right)=i, \forall n \in \mathbb{N}, \forall a_{(n)} \neq 0\right)$.

Daqui em diante, $a(z)$ e $b(w)$ serão homogêneos salvo menção explícita em contrário.

Teorema 2.7. Valem as seguintes igualdades:

(i) $a(z) b(w)=\left[a(z)_{-}, b(w)\right]+: a(z) b(w)$ :

(ii) $(-1)^{p(a) p(b)} b(w) a(z)=-\left[a(z)_{+}, b(w)\right]+: a(z) b(w):$ 
Demonstração. Temos que:

$\left[a(z)_{-}, b(w)\right]=a(z)_{-} b(w)-(-1)^{p(a) p(b)} b(w) a(z)_{-}$

$: a(z) b(w):=a(z)_{+} b(w)+(-1)^{p(a) p(b)} b(w) a(z)_{-}$

$\operatorname{Logo}\left[a(z)_{-}, b(w)\right]+: a(z) b(w):=a(z) b(w)$, o que prova $(i)$. Agora:

$-\left[a(z)_{+}, b(w)\right]=-a(z)_{+} b(w)+(-1)^{p(a) p(b)} b(w) a(z)_{+}$

$: a(z) b(w):=a(z)_{+} b(w)+(-1)^{p(a) p(b)} b(w) a(z)_{-}$

Assim,

$-\left[a(z)_{+}, b(w)\right]+: a(z) b(w):=(-1)^{p(a) p(b)} b(w) a(z)$,

o que prova (ii).

Teorema 2.8. Seja $c^{j}(w)=\sum_{n \in \mathbb{Z}} c_{(n)}^{j} w^{-n-1} \in \mathfrak{g}\left[\left[w, w^{-1}\right]\right]$ definido por (comparar com teorema 1.6):

$$
c^{j}(w)=\operatorname{Res}_{z}[a(z), b(w)] \cdot(z-w)^{j}, \forall j \in \mathbb{Z}_{+}
$$

São equivalentes:

(a) a(z) e b(z) são mutuamente locais.

(b) $[a(z), b(w)]=\sum_{j=0}^{N-1} \partial_{w}^{(j)} \delta(z-w) c^{j}(w)$

(c) $\left[a(z)_{-}, b(w)\right]=\sum_{j=0}^{N-1}\left(i_{z, w} \frac{1}{(z-w)^{j+1}}\right) c^{j}(w)$

$$
\left[a(z)_{+}, b(w)\right]=\sum_{j=0}^{N-1}\left(i_{w, z} \frac{1}{(z-w)^{j+1}}\right) c^{j}(w)
$$

(d) $a(z) b(w)=\sum_{j=0}^{N-1}\left(i_{z, w} \frac{1}{(z-w)^{j+1}}\right) c^{j}(w)+: a(z) b(w)$ :

$(-1)^{p(a) p(b)} b(w) a(z)=\sum_{j=0}^{N-1}\left(i_{w, z} \frac{1}{(z-w)^{j+1}}\right) c^{j}(w)+: a(z) b(w):$ 
(e) $\left[a_{(m)}, b_{(n)}\right]=\sum_{j=0}^{N-1}\left(\begin{array}{c}m \\ n\end{array}\right) c_{(m+n+j)}^{j}$, onde $m, n \in \mathbb{Z}$.

(f) $\left[a_{(m)}, b(w)\right]=\sum_{j=0}^{N-1}\left(\begin{array}{c}m \\ n\end{array}\right) c^{j}(w) w^{m-j}$, onde $m, n \in \mathbb{Z}$.

(g) $\left[a_{(m)}, b_{(n)}\right]=\sum_{j=0}^{N-1} p_{j}(m) d_{m+n}^{j}$, onde $p_{j}(x)$ é um polinômio e $d_{k}^{j}$ são elementos de $\mathfrak{g}$.

(h) $a(z) b(w)=\left[a(z)_{-}, b(w)\right]=\left(\frac{1}{(z-w)^{N}}\right) c(z, w)$

$$
(-1)^{p(a) p(b)} b(w) a(z)=\left(\frac{1}{(z-w)^{N}}\right) c(z, w)
$$

para alguma distribuição formal $c(z, w)$.

Demonstração. Provemos que $(a) \Leftrightarrow(b) \Leftrightarrow(c) \Leftrightarrow(d)$, que $(b) \Leftrightarrow(e) \Leftrightarrow(f)$, que $(e) \Leftrightarrow(g)$ e que $(d) \Rightarrow(h) \Rightarrow(a)$. Temos:

$(z-w)^{N} a(z, w)=0 \Leftrightarrow a(z, w)=\sum_{j=0}^{n-1} c^{j}(w) \partial_{w}(j) \delta(z-w)$,

onde $c^{j}(w)=\operatorname{Res}_{z} a(z, w) \cdot(z-w)^{j}$.

Basta tomar $a(z, w)=[a(z), b(w)]$ e assim $(a) \Leftrightarrow(b)$. Agora:

$\partial_{w}^{(j)} \delta(z-w)=i_{z, w} \frac{1}{(z-w)^{j+1}}-i_{w, z} \frac{1}{(z-w)^{j+1}}$

Assim,

$i_{z, w} \frac{1}{(z-w)^{j+1}}=\left(\partial_{w}^{(j)} \delta(z-w)\right)^{(-z)}$

e também

$\left[a(z)_{-}, b(w)\right]=[a(z), b(w)]^{-(z)}$

Logo: 


$$
\begin{aligned}
{\left[a(z)_{-}, b(w)\right] } & =\left(\sum_{j=0}^{N-1} \partial_{w}^{(j)} \delta(z-w) c^{j}(w)\right)^{-(z)} \\
& =\left(\sum_{j=0}^{N-1} \partial_{w}^{(j)} \delta(z-w)^{-(z)} c^{j}(w)\right) \\
& =\sum_{j=0}^{N-1}\left(i_{z, w} \frac{1}{(z-w)^{j+1}}\right) c^{j}(w)
\end{aligned}
$$

Analogamente,

$$
-\left[a(z)_{+}, b(w)\right]=\sum_{j=0}^{N-1}\left(i_{w, z} \frac{1}{(z-w)^{j+1}}\right) c^{j}(w)
$$

Logo $(b) \Rightarrow(c)$. Claramente $(c) \Rightarrow(b), \operatorname{logo}(b) \Leftrightarrow(c)$. Agora segue do Teorema 2.7 que $(c) \Leftrightarrow(d)$. Para provar que $(b) \Leftrightarrow(e)$, basta observar que:

$$
\begin{aligned}
{[a(z), b(w)] } & =\left[\sum_{m \in \mathbb{Z}} a_{(m)} z^{-m-1}, \sum_{n \in \mathbb{Z}} b_{(n)} w^{-n-1}\right] \\
& =\sum_{m, n \in \mathbb{Z}}\left[a_{(m)}, b_{(n)}\right] z^{-m-1} w^{-n-1}
\end{aligned}
$$

Assim, se valer $(e)$, então:

$$
\begin{aligned}
{[a(z), b(w)] } & =\sum_{m, n \in \mathbb{Z}} \sum_{j=0}^{N-1}\left(\begin{array}{c}
m \\
j
\end{array}\right) c_{(m+n-j)}^{j} \cdot z^{-m-1} \cdot w^{-n-1} \\
& =\sum_{m, n \in \mathbb{Z}} \sum_{j=0}^{N-1}\left(\begin{array}{c}
m \\
j
\end{array}\right) c_{(m+n-j)}^{j} \cdot w^{-m-n+j-1} \cdot w^{m-j} \cdot z^{-m-1} \\
& =\left(\sum_{j=0}^{N-1} \sum_{m \in \mathbb{Z}}\left(\begin{array}{c}
m \\
j
\end{array}\right) w^{m-j} z^{-m-1}\right) \cdot\left(\sum_{n_{1} \in \mathbb{Z}} c_{\left(n_{1}\right)}^{j} w^{-n_{1}-1}\right) \\
& =\left(\sum_{j=0}^{N-1} \partial_{w}^{(j)} \delta(z-w)\right) \cdot\left(\sum_{n_{1} \in \mathbb{Z}} c_{\left(n_{1}\right)}^{j} w^{-n_{1}-1}\right) \\
& =\sum_{j=0}^{N-1} \partial_{w}^{(j)} \delta(z-w) \cdot c^{j}(w)
\end{aligned}
$$

Logo $(e) \Rightarrow(b)$. A prova de que $(b) \Rightarrow(e)$ segue a mesma idéia. Assim 
temos $(b) \Leftrightarrow(e)$. Agora $(e) \Leftrightarrow(f)$, pois:

$$
\begin{aligned}
{\left[a_{(m)}, b(w)\right] } & =\left[a_{(m)}, \sum_{n \in \mathbb{Z}} b_{(n)} w^{-n-1}\right] \\
& =\sum_{n \in \mathbb{Z}}\left[a_{(m)}, b_{(n)}\right] w^{-n-1}
\end{aligned}
$$

Logo, se valer $(e)$, então:

$$
\begin{aligned}
{\left[a_{(m)}, b(w)\right] } & =\sum_{n \in \mathbb{Z}} \sum_{j=0}^{N-1}\left(\begin{array}{c}
m \\
j
\end{array}\right) c_{(m+n-j)}^{j} w^{-n-1} \\
& =\sum_{j=0}^{N-1}\left(\begin{array}{c}
m \\
j
\end{array}\right)\left(\sum_{n \in \mathbb{Z}} c_{(m+n-j)}^{j} w^{-m-n+j-1}\right) \cdot w^{m-j} \\
& =\sum_{j=0}^{N-1}\left(\begin{array}{c}
m \\
j
\end{array}\right) c^{j}(w) w^{m-j}
\end{aligned}
$$

logo valerá $(f)$. Agora que $(f) \Rightarrow(e)$ segue a mesma idéia. Logo $(e) \Leftrightarrow(f)$. Para $(e) \Leftrightarrow(g)$, é suficiente observar que qualquer polinômio é uma combinação linear de coeficientes binomiais. Finalmente, mostremos que $(d) \Rightarrow(h) \Rightarrow(a)$. Temos:

$$
\begin{aligned}
a(z) b(w) & =\sum_{j=0}^{N-1}\left(i_{z, w} \frac{1}{(z-w)^{j+1}}\right) \cdot c^{j}(w)+: a(z) b(w): \\
& =\sum_{j=0}^{n-1} c^{j}(w) \cdot(z-w)^{N-j-1} \cdot i_{z, w} \frac{1}{(z-w)^{N}} \\
& +: a(z) b(w): \cdot(z-w)^{N} \cdot i_{z, w} \frac{1}{(z-w)^{N}} \\
& =\left(\sum_{j=0}^{N-1} c^{j}(w) \cdot(z-w)^{N-j-1}+: a(z) b(w):(z-w)^{N}\right) \cdot i_{z, w} \frac{1}{(z-w)^{N}}
\end{aligned}
$$

Agora definamos:

$$
\begin{aligned}
& c(z, w)=\sum_{j=0}^{N-1} c^{j}(w) \cdot(z-w)^{N-j-1}+: a(z) b(w):(z-w)^{N} . \\
& \operatorname{Logo} a(z) b(w)=\left(i_{z, w} \frac{1}{(z-w)^{N}}\right) \cdot c(z, w), \text { e assim }(d) \Rightarrow(h) .
\end{aligned}
$$


Finalmente:

$$
\begin{aligned}
{[a(z), b(w)] } & =a(z) b(w)-(-1)^{p(a) p(b)} b(w) a(z) \\
& =\left(i_{z, w} \frac{1}{(z-w)^{N}}-i_{w, z} \frac{1}{(z-w)^{N}}\right) c(z, w) \\
& =\partial_{w}^{(N-1)} \delta(z-w) c(z, w)
\end{aligned}
$$

Portanto,

$$
\begin{aligned}
(z-w)^{N}[a(z), b(w)] & =(z-w)^{N} \partial_{w}^{(N-1)} \delta(z-w) c(z, w) \\
& =0 \cdot c(z, w) \\
& =0
\end{aligned}
$$

Logo $(h) \Rightarrow(a)$, o que prova o teorema.

Denota-se a primeira das relações do teorema anterior, ítem $(d)$ por:

$$
a(z) b(w)=\sum_{j=0}^{N-1} \frac{c^{j}(w)}{(z-w)^{j+1}}+: a(z) b(w):
$$

ou simplesmente:

$$
a(z) b(w) \sim \sum_{j=0}^{N-1} \frac{c^{j}(w)}{(z-w)^{j+1}}
$$

Estas expressões são chamadas de $O P E^{\prime} s$ ("operator product expansion"). Pelo teorema, a parte singular da OPE, definida por

$$
\sum_{j=0}^{N-1}\left(i_{z, w} \frac{1}{(z-w)^{j+1}} c^{j}(w)\right)
$$

contém todos os colchetes de todos os coeficientes de distribuições formais mutuamente locais $a(z)$ e $b(z)$. Esta é a razão pela qual desenvolve-se técnicas para calcular as $O P E^{\prime} s$.

Definição 2.9. Para todo $n \in \mathbb{Z}_{+}$, define-se o n-ésimo produto de a(z) e $b(z)$ como: 
(i) $a(w)_{(n)} b(w)=\operatorname{Res}_{z}[a(z), b(w)](z-w)^{n}$

(ii) $a(w)_{n} b(w)=\operatorname{Res}_{z} a(z) b(w)(z-w)^{n}$

Teorema 2.10. Se $a(z)$ e $b(z)$ são mutuamente locais, então valem as seguintes relações:

(i) $a(z) b(w)=\sum_{j=0}^{N-1} \frac{a(w)_{(j)} b(w)}{(z-w)^{j+1}}+: a(z) b(w):$

(ii) $[a(z), b(w)]=\sum_{j \in \mathbb{Z}_{+}}\left(a(w)_{(j)} b(w)\right) \partial_{w}^{(j)} \delta(z-w)$.

Demonstração. Basta definir $a(z, w)=[a(z), b(w)]$. Assim, tem-se:

$$
\begin{aligned}
c^{j}(w) & =\operatorname{Res}_{z} a(z, w) \cdot(z-w)^{j} \\
& =\operatorname{Res}_{z}[a(z), b(w)] \cdot(z-w)^{j} \\
& =a(w)_{(j)} b(w)
\end{aligned}
$$

O resultado segue pelo teorema anterior.

\section{$2.3 \mathrm{O} \quad \lambda$-produto e o $\lambda$-colchete de duas distribuições formais}

Um método eficiente de se estudar as $O P E^{\prime} s$ é através de sua transformada formal de Fourier, o que motiva a seguinte definição:

Definição 2.11. Seja U uma álgebra (por enquanto, não necessariamente associativa ou de Lie). Define-se o $\lambda$-produto $a(w)_{\lambda} b(w)$ de duas distribuições formais $U$-valuadas $a(w)$ e $b(w)$ como sendo:

$$
a(w)_{\lambda} b(w)=F_{z, w}^{\lambda}(a(z) b(w))=\sum_{n=0}^{\infty} \lambda^{(n)}\left(a(w)_{n} b(w)\right)
$$

onde a segunda igualdade é conseqüência imediata da definição de $F_{z, w}$.

Teorema 2.12. São verdadeiras as seguintes igualdades:

(i) $F_{z, w}^{\lambda} F_{x, w}^{\mu} a(z)(b(x) c(w))=a(w)_{\lambda}\left(b(w)_{\mu} c(w)\right)$ 
(ii) $F_{z, w}^{\lambda} F_{x, w}^{\mu}(a(z) b(x)) c(w)=\left(a(w)_{\lambda} b(w)\right)_{\lambda+\mu} c(w)$

(iii) $\left(\partial_{w} a(w)\right)_{\lambda} b(w)=-\lambda \cdot a(w)_{\lambda} b(w)$

$(i v) a(w)_{\lambda} \partial_{w} b(w)=\left(\lambda+\partial_{w}\right)\left(a(w)_{\lambda} b(w)\right)$

(v) $b(w)_{\lambda}^{\circ} a(w)=a(w)_{-\lambda-\partial_{w}} b(w)$

onde $a(w) \underset{\lambda}{\circ} b(w)$ denota o $\lambda$-produto da álgebra $U^{\circ p}$ (que nada mais é do que $U$ com a multiplicação oposta $a \underset{\lambda}{\circ} b=b \cdot a)$

Demonstração. Tem-se que:

$$
\begin{aligned}
a(w)_{\lambda}\left(b(w)_{\mu} c(w)\right) & =F_{z, w}^{\lambda}\left(a(z)\left(b(w)_{\mu} c(w)\right)\right. \\
& =F_{z, w}^{\lambda}\left(a(z)\left(F_{x, w}^{\mu}(b(x) c(w))\right)\right. \\
& =F_{z, w}^{\lambda} F_{x, w}^{\mu} a(z)(b(x) c(w))
\end{aligned}
$$

Agora, usando o Teorema 1.9, segue que:

$$
\begin{aligned}
F_{z, w}^{\lambda} F_{x, w}^{\mu}(a(z) b(x)) c(w) & =F_{x, w}^{\lambda+\mu} F_{z, x}^{\lambda}(a(z) b(x)) c(w) \\
& =F_{x, w}^{\lambda+\mu}\left(a(x)_{\lambda} b(x)\right) c(w) \\
& =\left(a(w)_{\lambda} b(w)\right)_{\lambda+\mu} c(w)
\end{aligned}
$$

e também que:

$$
\begin{aligned}
&\left(\partial_{w} a(w)\right)_{\lambda} b(w)= F_{z, w}^{\lambda}\left(\partial_{z} a(z) b(w)\right) \\
& \stackrel{T .1 .7(i v)}{=}-\lambda F_{z, w}^{\lambda} a(z) b(w) \\
&=-\lambda a(w)_{\lambda} b(w) \\
& a(w)_{\lambda} \partial_{w} b(w)=F_{z, w}^{\lambda}\left(a(z) \partial_{w} b(w)\right) \\
& \stackrel{T .1 .7(v)}{=} F_{z, w}^{-\lambda-\partial_{w}} a(w) \partial_{z} b(z) \\
&=F_{z, w}^{-\lambda-\partial_{w}} \partial_{z}(a(w) b(z)) \\
& \stackrel{T .1 .7(i v)}{=}-\left(-\lambda-\partial_{w}\right) F_{z, w}^{-\lambda-\partial_{w}} a(w) b(z) \\
& \stackrel{T .1 .7(v)}{=}\left(\lambda+\partial_{w}\right) F_{z, w}^{a}(z) b(w) \\
&=\left(\lambda+\partial_{w}\right)\left(a(w)_{\lambda} b(w)\right)
\end{aligned}
$$




$$
\begin{aligned}
b(w) \underset{\lambda}{\circ} a(w) & =F_{z, w}^{\lambda}(b(z) \underset{\lambda}{\circ} a(w)) \\
& =F_{z, w}^{\lambda}(a(w) b(z)) \\
& \stackrel{T .1 .7(v)}{=} F_{z, w}^{-\lambda-\partial_{w}} a(z) b(w) \\
& =a(w)_{-\lambda-\partial_{w}} b(w)
\end{aligned}
$$

Definição 2.13. Seja U uma superálgebra de Lie. Define-se o $\lambda$-colchete de $a(w)$ e $b(w)$ como sendo:

$$
\left[a(w)_{\lambda} b(w)\right]=\sum_{m=0}^{\infty} \lambda^{(m)}\left(a(w)_{(m)} b(w)\right)
$$

Teorema 2.14. Sejam $a(w), b(w)$ e $c(w)$ distribuições formais $\mathfrak{g}$-valuadas, onde $\mathfrak{g}$ é uma superálgebra de Lie. Então valem as seguintes igualdades:

(i) $\left[a(w)_{\lambda} b(w)\right]=-(-1)^{p(a) p(b)}\left[b(w)_{-\lambda-\partial_{w}} a(w)\right]$

(ii) $\left[a(w)_{\lambda}\left[b(w)_{\mu} c(w)\right]\right]=\left[\left[a(w)_{\lambda} b(w)\right]_{\lambda+\mu} c(w)\right]$

$$
+(-1)^{p(a) p(b)}\left[b(w)_{\mu}\left[a(w)_{\lambda} c(w)\right]\right]
$$

Demonstração. Temos que:

$$
\begin{aligned}
{[b(w), a(z)] } & =b(w) a(z)-(-1)^{p(a) p(b)} a(z) b(w) \\
& =-(-1)^{p(a) p(b)}[a(z), b(w)]
\end{aligned}
$$

Desta forma, temos que:

$$
\begin{aligned}
{\left[a(w)_{\lambda} b(w)\right] } & =\sum_{m=0}^{\infty} \lambda^{(m)}\left(a(w)_{\lambda} b(w)\right) \\
& =\sum_{m=0}^{\infty} \lambda^{(m)} \operatorname{Res}_{z}[a(z), b(w)] \cdot(z-w)^{m} \\
& =-(-1)^{p(a) p(b)} \sum_{m=0}^{\infty} \lambda^{(m)} \operatorname{Res}_{z}[b(w), a(z)] \cdot(z-w)^{m}
\end{aligned}
$$

Agora $b(w)_{-\lambda-\partial_{w}} a(w)=a(w) \underset{\lambda}{\circ} b(w)$. Logo: 


$$
\begin{aligned}
{\left[b(w)_{-\lambda-\partial_{w}} a(w)\right] } & =\left[a(w)_{\lambda}^{\circ} b(w)\right] \\
& =\sum_{m=0}^{\infty} \lambda^{(m)} \operatorname{Res}_{z}[b(w), a(z)] \cdot(z-w)^{m}
\end{aligned}
$$

Portanto,

$$
\begin{aligned}
{\left[a(w)_{\lambda} b(w)\right] } & =-(-1)^{p(a) p(b)} \sum_{m=0}^{\infty} \lambda^{(m)} \operatorname{Res}_{z}[b(w), a(z)] \cdot(z-w)^{m} \\
& =-(-1)^{p(a) p(b)}\left[b(w)_{-\lambda-\partial_{w}} a(w)\right]
\end{aligned}
$$

o que prova (i). Agora:

$$
\begin{aligned}
& {\left[a(w)_{\lambda}\left[b(w)_{\mu} c(w)\right]\right]=F_{z, w}^{\lambda} F_{x, w}^{\mu}[a(z),[b(x), c(w)]]} \\
& \stackrel{(\star)}{=} F_{z, w}^{\lambda} F_{x, w}^{\mu}\left([[a(z), b(x)], c(w)]+(-1)^{p(a) p(b)}[b(x),[a(z), c(w)]]\right) \\
& =F_{x, w}^{\lambda+\mu} F_{z, x}^{\lambda}\left([[a(z), b(x)], c(w)]+(-1)^{p(a) p(b)} F_{z, w}^{\lambda} F_{x, w}^{\mu}[b(x),[a(z), c(w)]]\right) \\
& =\left[\left[a(w)_{\lambda} b(w)\right]_{\lambda+\mu} c(w)\right]+(-1)^{p(a) p(b)}\left[b(w)_{\mu}\left[a(w)_{\lambda} c(w)\right]\right]
\end{aligned}
$$

onde $(\star)$ vale pela superidentidade de Jacobi. Isto prova $(i i)$.

Teorema 2.15. Valem as seguintes igualdades:

(i) $\partial a(w)_{n} b(w)=-n \cdot a(w)_{n-1} b(w)$

(ii) $a(w)_{n} \partial b(w)=\partial\left(a(w)_{n} b(w)\right)+n \cdot a(w)_{n-1} b(w)$

(iii) $a(w)_{(n)} b(w)=-(-1)^{p(a) p(b)} \sum_{j=0}^{\infty}(-1)^{j+n} \partial_{w}^{(j)}\left(b(w)_{(n+j)} a(w)\right)$, desde que $a(w)$ e $b(w)$ sejam mutuamente locais.

$$
\begin{aligned}
(i v) a(w)_{(m)}\left(b(w)_{(n)} c(w)\right)= & \sum_{j=0}^{\infty}\left(\begin{array}{c}
m \\
j
\end{array}\right)\left(a(w)_{(j)} b(w)\right)_{(m+n-j)} c(w) \\
& +(-1)^{p(a) p(b)} b(w)_{(n)}\left(a(w)_{(m)} c(w)\right)
\end{aligned}
$$

Demonstração. Temos que:

$$
\begin{aligned}
0 & =\operatorname{Res}_{z} \partial_{z}\left((z-w)^{n} a(z) b(w)\right) \\
& =\operatorname{Res}_{z}\left[n \cdot(z-w)^{n-1} a(z) b(w)+(z-w)^{n} \partial_{z} a(z) b(w)\right] \\
& =n \cdot a(w)_{n-1} b(w)+\partial a(w)_{n} b(w)
\end{aligned}
$$


Logo, $\partial a(w)_{n} b(w)=-n \cdot a(w)_{n-1} b(w)$. Agora

$$
\begin{aligned}
\partial\left(a(w)_{n} b(w)\right) & =\partial_{w}\left(\operatorname{Res}_{z}(z-w)^{n} a(z) b(w)\right) \\
& =\operatorname{Res}_{z}\left(-n \cdot(z-w)^{n-1} a(z) b(w)+(z-w)^{n} a(z) b(w)\right) \\
& =-n \cdot a(w)_{n-1} b(w)+a(w)_{n} \partial b(w)
\end{aligned}
$$

Logo temos (ii). Agora sabemos que

$$
\begin{aligned}
& \begin{aligned}
b(w)_{(n)} a(w) & =\operatorname{Res}_{z}[b(z), a(w)] \cdot(z-w)^{n} \\
& =c^{n}(w)
\end{aligned} \\
& \text { e que } \\
& \begin{aligned}
a(w)_{(n)} b(w) & =\operatorname{Res}_{z}[a(z), b(w)] \cdot(z-w)^{n} \\
& =-(-1)^{p(a) p(b)} \operatorname{Res}_{z}[b(w), a(z)] \cdot(z-w)^{n} \\
& =-(-1)^{p(a) p(b)} \tilde{c}^{n}(w)
\end{aligned}
\end{aligned}
$$

Agora

$\tilde{c}^{n}(w)=\sum_{j=0}^{\infty}(-1)^{j+n} \partial_{w}^{(j)}\left(b(w)_{(n+j)} a(w)\right)$. Logo, temos:

$a(w)_{(n)} b(w)=-(-1)^{p(a) p(b)} \sum_{j=0}^{\infty}(-1)^{j+n} \partial_{w}^{(j)}\left(b(w)_{(n+j)} a(w)\right)$

Finalmente, provemos $(i v)$ :

$$
\begin{aligned}
& a(w)_{(m)}\left(b(w)_{(n)} c(w)\right)=\operatorname{Res}_{x}\left[a(x), b(w)_{(n)} c(w)\right] \cdot(x-w)^{m} \\
& =\operatorname{Res}_{x} \operatorname{Res}_{z}[a(x),[b(z), c(w)]] \cdot(z-w)^{n}(x-w)^{m} \\
& \stackrel{(\star)}{=} \operatorname{Res}_{x}\left(\left(\operatorname{Res}_{z}[[a(x), b(z)], c(w)] \cdot(z-w)^{n}\right) \cdot(x-w)^{m}\right) \\
& \left.+(-1)^{p(a) p(b)} \operatorname{Res}_{x}\left(\operatorname{Res}_{z}[b(z),[a(x), c(w)]] \cdot(z-w)^{n}\right) \cdot(x-w)^{m}\right) \\
& \left.=\operatorname{Res}_{x}\left(\operatorname{Res}_{z}[[a(x), b(z)], c(w)] \cdot(z-w)^{n}\right) \cdot(x-w)^{m}\right) \\
& +(-1)^{p(a) p(b)} b(w)_{(n)}\left(a(w)_{(m)} c(w)\right)
\end{aligned}
$$


onde $(\star)$ vale pela superidentidade de Jacobi. Agora basta provar que:

$$
\begin{aligned}
& \sum_{j=0}^{m}\left(\begin{array}{c}
m \\
j
\end{array}\right)\left(a(w)_{(j)} b(w)\right)_{(m+n-j)} c(w)= \\
& =\operatorname{Res}_{x} \operatorname{Res}_{z}[[a(x), b(z)], c(w)] \cdot(z-w)^{n} \cdot(x-w)^{m}
\end{aligned}
$$

De fato,

$$
\begin{aligned}
& \sum_{j=0}^{m}\left(\begin{array}{c}
m \\
j
\end{array}\right)\left(a(w)_{(j)} b(w)\right)_{(m+n-j)} c(w)=\sum_{j=0}^{m}\left(\begin{array}{c}
m \\
j
\end{array}\right) a(w)_{(j)}\left(b(w)_{(m+n-j)} c(w)\right) \\
& =\sum_{j=0}^{m}\left(\begin{array}{c}
m \\
j
\end{array}\right) \operatorname{Res}_{z}\left[a(z)_{(j)} b(z), c(w)\right] \cdot(z-w)^{m+n-j} \\
& =\operatorname{Res}_{x} \operatorname{Res}_{z} \sum_{j=0}^{m}\left(\begin{array}{c}
m \\
j
\end{array}\right)[a(x),[b(z), c(w)]] \cdot(x-z)^{j}(z-w)^{m-j} \cdot(z-w)^{n} \\
& \stackrel{(\star)}{=} \operatorname{Res}_{x} \operatorname{Res}_{z}[[a(x), b(z)], c(w)] \cdot(x-w)^{m} \cdot(z-w)^{n}
\end{aligned}
$$

onde $(\star)$ vale pois:

$$
(x-w)^{m}=((x-z)+(z-w))^{m}=\sum_{j=0}^{m}\left(\begin{array}{c}
m \\
j
\end{array}\right) \cdot(x-z)^{j} \cdot(z-w)^{m-j}
$$

o que prova o teorema.

Corolário 2.16. Seja $\mathfrak{g}$ uma superálgebra de Lie. Temos:

(i) Se $a(z)$ e $b(z)$ são distribuições formais $\mathfrak{g}$-valuadas, então $\left[a_{(0)}, b(z)\right]=0$ se, e somente se, $a(z)_{(0)} b(z)=0$.

(ii) se a(z) é uma distribuição formal $\mathfrak{g}$-valuada ímpar, então $a_{(0)}^{2}=0$ se, e somente se, $\operatorname{Res}_{z} a(z)_{(0)} a(z)=0$.

(iii) Seja A um espaço g-valuado que consiste de distribuições formais mutuamente locais em $w$ que seja $\partial$-invariante e fechado com respeito a todos os n-ésimos produtos, com $n \in \mathbb{Z}_{+}$. Então com relação ao 0-ésimo produto, $\partial A$ é um ideal bilateral de $A$ e $A / \partial A$ é uma superálgebra de Lie. Portanto o 0-ésimo produto define em $A$ uma estrutura de um $A / \partial A$-módulo à esquerda.

Demonstração. Temos que 


$$
\begin{aligned}
a(z)_{(0)} b(z) & =\operatorname{Res}_{x}[a(x), b(z)] \cdot(x-z)^{0} \\
& =\left[\operatorname{Res}_{x} a(x), b(z)\right] \\
& =\left[a_{(0)}, b(z)\right]
\end{aligned}
$$

o que prova $(i)$. Agora para provar $(i i)$, basta provar que $\operatorname{Res}_{z} a(z)_{(0)} a(z)=$ $2 \cdot a_{(0)}^{2}$. Temos:

$$
\begin{aligned}
\operatorname{Res}_{z} a(z)_{(0)} a(z) & =\operatorname{Res}_{z} \operatorname{Res}_{x}[a(x), a(z)] \cdot(x-z)^{0} \\
& =\left[\operatorname{Res}_{x} a(x), \operatorname{Res}_{z} a(z)\right] \\
& =\left[a_{(0)}, a_{(0)}\right] \\
& =a_{(0)} a_{(0)}-(-1)^{p(a) p(a)} a_{(0)} a_{(0)} \\
& =a_{(0)} a_{(0)}+a_{(0)} a_{(0)} \\
& =2 \cdot a_{(0)}^{2}
\end{aligned}
$$

já que $a(z)$ é ímpar. Agora provemos (iii). Temos que $\partial A$ é um ideal bilateral, pois:

(1) $0 \in \partial A$

(2) se $a, b \in \partial A$, então $(a-b) \in \partial A$

$(3)(\partial A)_{(0)} A=0 \subset \partial A$

De fato, (1) e (2) são verdadeiros e (3) vale, pois:

$\partial a(w)_{n} b(w)=-n \cdot a(w)_{n-1} b(w)$

Logo $\partial a(w)_{0} b(w)=0$, e assim $\partial A_{0} A=0 \subset \partial A$. Agora $\partial A$ é um ideal à esquerda, pois:

$a(w)_{n} \partial b(w)=\partial\left(a(w)_{n} b(w)\right)+n \cdot a(w)_{n-1} b(w)$

implica que

$a(w)_{0} \partial b(w)=\partial\left(a(w)_{0} b(w)\right) \in \partial A$

Logo $A_{0} \partial A \subset \partial A, \log A_{(0)} \partial A \subset \partial A$. Assim, $\partial A$ é um ideal bilateral. Agora temos que: 


$$
\begin{aligned}
& a(w)_{(0)} b(w)=-(-1)^{p(a) p(b)} \sum_{j=0}^{\infty}(-1)^{j} \partial_{w}^{(j)}\left(b(w)_{(0+j)} a(w)\right) \\
& =-(-1)^{p(a) p(b)} b(w)_{(0)} a(w)+\partial_{w}\left(-(-1)^{p(a) p(b)} \sum_{j=1}^{\infty} \frac{(-1)^{j}}{j !} \partial_{w}^{j-1}\left(b(w)_{(j)} a(w)\right)\right.
\end{aligned}
$$

e como $\partial_{w}\left(-(-1)^{p(a) p(b)} \sum_{j=1}^{\infty} \frac{(-1)^{j}}{j !} \partial_{w}^{j-1}\left(b(w)_{(j)} a(w)\right) \in \partial A\right.$, segue que:

$a(w)_{(0)} b(w)=-(-1)^{p(a) p(b)} b(w)_{(0)} a(w) \bmod \partial A$

Assim, o 0-ésimo produto induz um colchete anti-supersimétrico em $A / \partial A$ (já que em $A / \partial A$, temos que $a(w)_{(0)} b(w)=-(-1)^{p(a) p(b)} b(w)_{(0)} a(w)$ ). Agora a superidentidade de Jacobi em $A / \partial A$ é válida, pois:

$$
\begin{aligned}
& a(w)_{(0)}\left(b(w)_{(0)} c(w)\right)= \\
& =\sum_{j=0}^{0}\left(\begin{array}{l}
0 \\
j
\end{array}\right)\left(a(w)_{(j)} b(w)\right)_{(0+0-j)} c(w)+(-1)^{p(a) p(b)} b(w)_{(0)}\left(a(w)_{(0)} c(w)\right)= \\
& =\left(a(w)_{(0)} b(w)\right)_{(0)} c(w)+(-1)^{p(a) p(b)} b(w)_{(0)}\left(a(w)_{(0)} c(w)\right)
\end{aligned}
$$

E isto prova que $A / \partial A$ é, de fato, uma superálgebra de Lie.

\subsection{Fórmula de Taylor}

Definição 2.17. Seja $a(z)=\sum_{n} a_{n} z^{n}$ uma distribuição formal. Define-se $i_{z, w} a(z-w)$ como sendo a distribuição formal em $z$ e $w$ dada por:

$$
i_{z, w} a(z-w)=\sum_{n} a_{n} \cdot i_{z, w}(z-w)^{n}
$$

Para simplificar a notação, considera-se esta distribuição formal como $a(z-w)$, de $z$ e $w$, no domínio $|z|>|w|$ (como já visto no Capítulo anterior).

Teorema 2.18. Seja a(z) uma distribuição formal. Então valem as seguintes igualdades:

(i) $a(z+w)=\sum_{j=0}^{\infty} \partial^{(j)} a(z) w^{j}$

(ii) $a(z)=\sum_{j=0}^{\infty} \partial^{(j)} a(w) \cdot(z-w)^{j}$. 
Demonstração. De $a(z)=\sum_{n} a_{n} z^{n}$, vem que $\partial^{(j)} a(z)=\sum_{n}\left(\begin{array}{c}n \\ j\end{array}\right) a_{n} z^{n-j}$ Assim,

$$
\begin{aligned}
a(z+w) & =\sum_{n} a_{n} \cdot(z+w)^{n} \\
& =\sum_{n} a_{n} \sum_{j=0}^{\infty}\left(\begin{array}{l}
n \\
j
\end{array}\right) z^{n-j} w^{j} \\
& =\sum_{j=0}^{\infty}\left(\sum_{n}\left(\begin{array}{l}
n \\
j
\end{array}\right) a_{n} z^{n-j}\right) w^{j} \\
& =\sum_{j=0}^{\infty} \partial^{(j)} a(w) \cdot(z-w)^{j}
\end{aligned}
$$

o que prova (i). Agora:

$$
\begin{aligned}
a(z) & =a(w+(z-w)) \\
& =\sum_{j=0}^{\infty} \partial^{(j)} a(w) \cdot(z-w)^{j}
\end{aligned}
$$

o que prova (ii).

Teorema 2.19. Seja a(z) uma distribuição formal e $N$ um inteiro nãonegativo. Então vale a igualdade:

$\partial_{w}^{N} \delta(z-w) \cdot a(z)=\partial_{w}^{N} \delta(z-w) \cdot \sum_{j=0}^{N} \partial^{(j)} a(w) \cdot(z-w)^{j}$

Demonstração. Basta provar que, para todo $f(z) \in \mathbb{C}\left[z, z^{-1}\right]$, temos:

$\left\langle\partial_{w}^{N} \delta(z-w) a(z), f(z)\right\rangle=\left\langle\partial_{w}^{N} \delta(z-w) \sum_{j=0}^{N} \partial^{(j)} a(w) \cdot(z-w)^{j}, f(z)\right\rangle$

Temos: 


$$
\begin{aligned}
\left\langle\partial_{w}^{N} \delta(z-w) a(z), f(z)\right\rangle & =\operatorname{Res}_{z} \partial_{z}^{N} \delta(z-w) a(z) f(z) \\
& =(-1)^{N} \operatorname{Res}_{z} \delta(z-w) \partial^{N}(a(z) f(z)) \\
& =(-1)^{N} \partial^{N}(a(w) f(w)) \\
& \stackrel{(\star)}{=}(-1)^{N} \sum_{j=0}^{N} \partial^{(j)} a(w)\left(\begin{array}{c}
N \\
j
\end{array}\right) \partial^{N-j} f(w)=\star
\end{aligned}
$$

onde $(\star)$ vale pela Regra de Leibnitz. Agora:

$\partial_{z}^{N}\left[(z-w)^{j} \cdot f(z)\right]=\sum_{i=0}^{\infty} \partial_{z}^{i}(z-w)^{j}\left(\begin{array}{c}N \\ i\end{array}\right) \partial^{N-i} f(z)$

Assim:

$\operatorname{Res}_{z} \delta(z-w) \partial_{z}^{N}\left[(z-w)^{j} f(z)\right]=\operatorname{Res}_{z} \sum_{i=0}^{\infty}\left(\begin{array}{c}N \\ i\end{array}\right) \delta(z-w) \partial_{z}^{i}(z-w)^{j} \cdot \delta^{N-i} f(z)$

Agora se $i>j$, então $\partial_{z}^{i}(z-w)^{j}=0$. Caso $i<j$, então:

$$
\begin{aligned}
\partial_{z}^{i}(z-w)^{j} & =i ! \cdot \partial_{z}^{j-i} \cdot(z-w)^{0} \\
& =0
\end{aligned}
$$

Desta forma, sobra apenas $i=j$. Por isso:

$$
\begin{aligned}
\star & =(-1)^{N} \sum_{j=0}^{N} \partial^{(j)} a(w) \operatorname{Res}_{z} \delta(z-w) \cdot \partial_{z}^{N}\left((z-w)^{j} \cdot f(z)\right) \\
& =\sum_{j=0}^{N} \partial^{(j)} a(w) \operatorname{Res}_{z} \partial_{z}^{N} \delta(z-w) \cdot(z-w)^{j} f(z) \\
& =\left\langle\partial_{w}^{N} \delta(z-w) \sum_{j=0}^{N} \partial^{(j)} a(w) \cdot(z-w)^{j}, f(z)\right\rangle
\end{aligned}
$$

\subsection{Exemplos de superálgebras de Lie}

O objetivo principal desta Seção é familiarizar o leitor com as principais superálgebras de Lie que serão utilizadas no decorrer deste trabalho. Destas, destaco as álgebras de Witt, Heisenberg e, principalmente, as álgebras de Virasoro e de Correntes. Sendo assim, começemos nosso estudo. 


\subsection{1 Álgebras de Witt}

Definição 2.20. Seja Vect $\mathbb{C}^{\times}$o espaço gerado pelos símbolos $\left\{t^{m} \partial_{t} / m \in \mathbb{Z}\right\}$ e seja o produto definido por:

$$
t^{m} \partial_{t} \cdot t^{n} \partial_{t}=(n-m) t^{m+n-1} \partial_{t}
$$

para todos os $m, n \in \mathbb{Z}$, e estenda-os por bilinearidade. Sendo assim (apenas mudando a notação), seja o colchete de Lie dado por:

$$
\left[t^{m} \partial_{t}, t^{n} \partial_{t}\right]=(n-m) t^{m+n-1} \partial_{t}
$$

A álgebra de Lie assim obtida é chamada álgebra de Witt (ou ainda, álgebra de Lie dos campos vetoriais algébricos sobre $\mathbb{C}^{\times}$). A álgebra de Lie dos campos vetoriais algébricos sobre $\mathbb{C}$, denotada por Vect $\mathbb{C}$, é a subálgebra de Vect $\mathbb{C}^{\times}$gerada por $t^{m} \partial_{t}$, com $m \geq 0$. Uma notação mais conveniente (embora menos versátil) para as álgebras de Witt é obtida repassando o gerador $-t^{m+1} \partial_{t}$ com o símbolo $L_{m}$, para todo $m \in \mathbb{Z}$. Assim:

$$
\begin{aligned}
{\left[L_{m}, L_{n}\right] } & =\left[-t^{m+1} \partial_{t},-t^{n+1} \partial_{t}\right] \\
& =(n-m) \cdot t^{m+n+1} \partial_{t} \\
& =(m-n) L_{m+n}
\end{aligned}
$$

para todo $m, n \in \mathbb{Z}$.

A álgebra de Witt pode também ser considerada como o espaço vetorial de derivações do anel dos polinômios de Laurent, com o colchete de Lie induzido pela composição do produto associativo. Este ponto de vista será bastante utilizado mais tarde. Uma derivação $\partial$ de uma álgebra $A$ é um endomorfismo que satisfaz a " regra do produto", isto é,

$$
\partial(a b)=\partial a b+a \partial b
$$

para todo $a, b \in A$. O primeiro exemplo de derivação de uma álgebra de Lie $\mathfrak{g}$ é a imagem do elemento $x$ pela sua representação adjunta, denotada por $a d(x)$, a qual é definida como:

$$
\operatorname{ad}(x)(y)=[x, y]
$$

\subsubsection{Extensões centrais}

Definição 2.21. Um elemento de uma álgebra de Lie $\mathfrak{g}$ é dito central se o seu colchete de Lie com qualquer outro elemento de $\mathfrak{g}$ é nulo. O centro de uma álgebra de Lie, denotado por $Z(\mathfrak{g})$, é a coleção de todos os elementos 
centrais.

Definição 2.22. Uma álgebra de Lie $\hat{\mathfrak{g}}$ é uma extensão central de uma álgebra de Lie $\mathfrak{g}$ se existe um homomorfismo sobrejetor $\varphi: \hat{\mathfrak{g}} \rightarrow \mathfrak{g}$ tal que Ker $\varphi$ é central. A dimensão desta extensão central é definida como sendo a dimensão do Ker $\varphi$ vista como um espaço vetorial. Extensões centrais são os objetos de maior interesse nesta Seção.

Definição 2.23. Seja $\mathfrak{g}$ uma álgebra de Lie sobre $\mathbb{C}$ e seja $c: \mathfrak{g} \times \mathfrak{g} \rightarrow \mathbb{C}$ tal que:

(a) c é bilinear

(b) c é anti-simétrica

(c) $c(x,[y, z])+c(y,[z, x])+c(z,[x, y])=0$,

para todo $x, y, z \in \mathfrak{g}$.

Estas formas são comumente chamadas de 2-cociclo, um nome proveniente da teoria de cohomologia.

Seja $K$ um símbolo, e $\hat{\mathfrak{g}}=\mathfrak{g} \oplus \mathbb{C} K$ um espaço vetorial. Então defina a aplicação bilinear $\widehat{[\cdot, \cdot]}: \hat{\mathfrak{g}} \times \hat{\mathfrak{g}} \rightarrow \hat{\mathfrak{g}}$ por:

$$
\widehat{[x, y]}=[x, y]+c(x, y) K \text { e por } \widehat{[\hat{\mathfrak{g}}, K]}=\widehat{[K, \hat{\mathfrak{g}}]}=0
$$

para todo $x, y \in \mathfrak{g}$. É imediato da natureza da forma $c$ que $\widehat{[\cdot, \cdot]}$ é um colchete de Lie. A álgebra de Lie $\hat{\mathfrak{g}}$ é uma extensão central unidimensional de $\mathfrak{g}$. Para verificar isto, seja $\varphi: \hat{\mathfrak{g}} \rightarrow \mathfrak{g}$ dada por $\left.\varphi\right|_{\mathfrak{g}}=I d_{\mathfrak{g}}$ e $\left.\varphi\right|_{k}=0$. Este é um homomorfismo sobrejetor de álgebra de Lie, como visto.

\subsection{3 Álgebras de Heisenberg}

Defina o colchete de Lie sobre $\mathbb{C}$ como sendo nulo. A álgebra de Laços sobre a álgebra de Lie é o anel dos polinômios de Laurent $\mathbb{C}\left[t, t^{-1}\right]$. Os polinômios de Laurent tem uma noção formal natural de derivação e resíduo, que são operações lineares dadas (respectivamente) por:

$$
\begin{aligned}
& \partial_{t} t^{m}=m \cdot t^{m-1} \\
& \operatorname{Res}_{t} t^{m}=\delta_{m,-1}
\end{aligned}
$$


para todo inteiro $m$. Agora considere a forma bilinear

$$
\begin{aligned}
\phi\left(t^{m}, t^{n}\right) & =-\operatorname{Res}_{t} t^{m} \partial_{t} t^{n} \\
& =-n \cdot \operatorname{Res}_{t} t^{m+n-1} \\
& =-n \cdot \delta_{m,-n} \\
& =m \cdot \delta_{m,-n}
\end{aligned}
$$

Uma rápida checagem revela que $\phi$ é anti-simétrica. A identidade de Jacobi é satisfeita, uma vez que o colchete de Lie em $\mathbb{C}\left[t, t^{-1}\right]$ é trivial. Desta forma, $\phi$ é um 2-cociclo.

A álgebra de Heisenberg $H$ sobre $\mathbb{C}$ é uma extensão central unidimensional de $\mathbb{C}\left[t, t^{-1}\right]$ associado à $\phi$. De forma abstrata, $H$ é gerado pelos símbolos $v_{m}$, indexado pelos inteiros, e um símbolo $K$, com colchete de Lie:

$$
\begin{aligned}
& {\left[v_{m}, v_{n}\right]=m \cdot \delta_{m-n} K ;} \\
& {\left[v_{m}, K\right]=0}
\end{aligned}
$$

para todos os inteiros $m, n$.

\subsubsection{A álgebra de Virasoro}

Aqui será construída uma extensão central da álgebra de Witt $W$, chamada álgebra de Virasoro. Defina uma forma bilinear $\phi: W \times W \rightarrow \mathbb{C}$ por:

$$
\phi\left(L_{m}, L_{n}\right)=\delta_{m,-n} \frac{m^{3}-m}{12}
$$

para todos os inteiros $m, n$ e extenda-os bilinearmente. Então:

$$
\begin{aligned}
\phi\left(L_{m}, L_{n}\right) & =\delta_{m,-n} \frac{(-n)^{3}-(-n)}{12} \\
& =-\delta_{n,-m} \frac{n^{3}-n}{12} \\
& =-\phi\left(L_{n}, L_{m}\right)
\end{aligned}
$$

e $\operatorname{assim} \phi$ é anti-simétrico. Com um pouco mais de trabalho, verifica-se que $\phi$ satisfaz a identidade de Jacobi e assim $\phi$ é um 2-cociclo. A álgebra de Virasoro sobre $\mathbb{C}$ é uma extensão central de $W$ com respeito à forma $\phi$. 
Temos:

$$
\left[L_{m}, L_{n}\right]=(m-n) L_{m+n}+\delta_{m,-n} \frac{m^{3}-m}{12} c
$$

para todos os inteiros $m, n$, onde o símbolo $c$ é definido de forma a ser central.

\subsubsection{O afinizador de Kac-Moody}

Seja $\mathfrak{g}$ uma álgebra de Lie sobre $K$. Uma forma bilinear $\tau: \mathfrak{g} \times \mathfrak{g} \rightarrow K$ é chamada invariante se:

$$
\tau([a, b], c])=\tau(a,[b, c])
$$

para todo $a, b, c \in \mathfrak{g}$.

Seja $\phi$ uma tal forma. Associe ao par $(\mathfrak{g}, \phi)$ uma extensão central unidimensional da álgebra de Laços $\mathfrak{g}\left[t, t^{-1}\right]$ associada a $\mathfrak{g}$, chamada de afinizador de Kac-Moody deste par. A construção é a seguinte:

Seja $\phi_{\tau}$ a forma bilinear em $\mathfrak{g}\left[t, t^{-1}\right]$ dada por:

$$
\phi_{\tau}\left(a t^{m}, b t^{n}\right)=m \cdot \delta_{m,-n} \cdot \tau(a, b)
$$

para todos os inteiros $m, n$ e todos os $a, b \in \mathfrak{g}$. É fácil ver que $\phi_{\tau}$ é antisimétrico. Assim, basta mostrar que $\phi_{\tau}$ safisfaz a identidade de Jacobi:

$$
\begin{aligned}
& \phi_{\tau}\left(a t^{m},\left[b t^{n}, c t^{p}\right]\right)+\phi_{\tau}\left(b t^{n},\left[c t^{p}, a t^{m}\right]\right)+\phi_{\tau}\left(c t^{p},\left[a t^{m}, b t^{n}\right]\right) \\
& =\delta_{m+n+p, 0}(m \cdot \tau(a,[b, c])+n \cdot \tau(b,[c, a])+p \cdot \tau(c,[a, b])) \\
& =\delta_{m+n+p, 0}(n \cdot \tau(b,[a, c])+(m+p) \tau(c,[a, b])) \\
& =n \cdot \tau(b,[a, c])-n \cdot \tau(b,[a, c]) \\
& =0
\end{aligned}
$$

já que $\tau$ é simétrico e invariante. Assim, $\phi_{\tau}$ é um 2-cociclo. Associe $(\mathfrak{g}, \tau)$ à extensão central unidimensional $\hat{\mathfrak{g}}$ de $\mathfrak{g}\left[t, t^{-1}\right]$ por $\phi_{\tau}$. Então $\hat{\mathfrak{g}}=\mathfrak{g} \oplus k K$, como espaço vetorial, com colchete de Lie:

$$
\left[a t^{m}, b t^{n}\right]=[a, b] t^{m+n}+m \cdot \delta_{m,-n} \tau(a, b) K
$$

para todos os inteiros $m, n$ e todos os $a, b \in \mathfrak{g}$, com o símbolo $K$ sendo central. Observe que a álgebra de Heisenberg é o afinizador de $(K, \tau) \operatorname{com} \tau$ sendo dado por $\tau(a, b)=a b$, para todo $a, b \in K$. 


\subsubsection{Afinizador de Clifford}

Seja $U=U_{\overline{0}} \oplus U_{\overline{1}}$ um espaço vetorial $\mathbb{Z}_{2}$-graduado sobre $\mathbb{C}$. Uma forma bilinear $\tau$ em $\mathrm{U}$ é dita anti-supersimétrica se:

$$
\tau(a, b)=-(-1)^{p(a)} \tau(b, a)
$$

para todos os elementos homogêneos não-nulos $a, b \in U$. Seja $\tau$ uma tal forma em $U$. Considere $U$ como uma superálgebra de Lie com o supercolchete de Lie definido como sendo o operador nulo. Seja $K$ um símbolo e $\widehat{U}=U\left[t, t^{-1}\right] \oplus k K$. Para todo $a t^{m}, b t^{n} \in U\left[t, t^{-1}\right]$, defina:

$$
\begin{aligned}
& {\left[\widehat{a t^{m}, b t^{n}}\right]=\tau(a, b) \cdot \delta_{m+n+1,0} \cdot K} \\
& {\left[\widehat{a t^{m}, K}\right]=0}
\end{aligned}
$$

Este colchete se estende para um supercolchete de Lie em $\widehat{U}$ donde chamase a superálgebra de Lie sobre ele obtida de afinizador de Clifford do par $(U, \tau)$

\subsection{7 Álgebras de Correntes}

Seja $\mathfrak{g}$ uma superálgebra de Lie com forma bilinear supersimétrica invariante $(\cdot \mid \cdot)$. Como visto, a álgebra de Laços associada a $\mathfrak{g}$ é a superálgebra de Lie sobre $\mathbb{C}$ dada por:

$$
\tilde{\mathfrak{g}}=\mathfrak{g}\left[t, t^{-1}\right]=\mathfrak{g} \underset{\mathbb{C}}{\otimes} \mathbb{C}\left[t, t^{-1}\right]
$$

$\operatorname{com} \mathbb{Z} / 2 \mathbb{Z}$-graduação estendida de $\mathfrak{g}$ por $p(t)=0$ (ou seja, $t$ é homogêneo de paridade nula) e relação de comutação:

$$
\left[a_{m}, b_{n}\right]=[a, b]_{m+n}
$$

onde $a_{m}$ significa $a \otimes t^{m}$. Note que $\tilde{\mathfrak{g}}$ é uma superálgebra de Lie de aplicações regulares de $\mathbb{C}^{*}$ em $\mathfrak{g}$. O afinizador do par $(\mathfrak{g},(\cdot \mid \cdot))$ é uma extensão central da álgebra de Laços $\tilde{\mathfrak{g}}$ por um centro unidimensional à direita $\mathbb{C} K$, isto é:

$$
\hat{\mathfrak{g}}=\tilde{\mathfrak{g}}+\mathbb{C} K
$$

com relação de comutatividade:

$$
\begin{aligned}
& {\left[a_{m}, b_{n}\right]=[a, b]_{m+n}+m \cdot(a \mid b) \delta_{m,-n} K} \\
& {[K, \hat{\mathfrak{g}}]=0}
\end{aligned}
$$


A superálgebra de Lie $\mathfrak{g}$ é usualmente chamada de álgebras de Correntes.

Observação 2.24. Se $\mathfrak{g}$ é uma álgebra de Lie simples de dimensão finita com a forma de Killing $(\cdot, \cdot)$, então $\hat{\mathfrak{g}}$ é conhecida como uma álgebra de Kac-Moody afim. Se $\mathfrak{g}$ é uma álgebra de Lie unidimensional com forma bilinear nãodegenerada, então recupera-se o exemplo da álgebra de Heisenberg. Agora também existe uma superextensão central natural do afinizador, chamado superafinizador, dado por:

$$
\hat{\mathfrak{g}}_{\text {super }}=\underset{\mathbb{C}}{\underset{\mathbb{C}}{\otimes}} \mathbb{C}\left[t, t^{-1}, \theta\right]+\mathbb{C} K
$$

onde $\theta^{2}=0$ e $p(\theta)=1$. Esta superálgebra de Lie é conhecida como superálgebra de Corrente. Para maiores detalhes, veja [1].

\subsection{8 Álgebra de Neveu-Schwarz}

A álgebra de Neveu-Schwarz é uma superálgebra de Lie gerada por elementos de paridade par $L_{m}$ e elementos de paridade ímpar $G_{r}$, onde $m \in \mathbb{Z}$ e $r \in \mathbb{Z}+\frac{1}{2}$, com elemento central $c$ satisfazendo as relações:

$$
\begin{aligned}
& {\left[L_{m}, L_{n}\right]=(m-n) L_{m+n}+\delta_{m,-n} \frac{m^{3}-m}{8} c} \\
& {\left[L_{m}, G_{r}\right]=\left(\frac{m}{2}-r\right) G_{m+r}} \\
& {\left[G_{r}, G_{s}\right]=G_{r} G_{s}+G_{s} G_{r}=2 L_{r+s}+\delta_{r,-s} \frac{\left(r^{2}-\frac{1}{4}\right)}{2} c}
\end{aligned}
$$

\subsection{Exemplos de superálgebras de Lie de distribuições formais}

Aqui será discutido um dos exemplos mais importantes de álgebras geradas por distribuições formais mutuamente locais, as álgebras de Correntes, mas para começar, será considerado um caso mais simples, a álgebra de Heisenberg $S$.

Esta é uma álgebra de Lie com elementos de base $\alpha_{n}(n \in \mathbb{Z})$ e $K$, com a seguinte relação de comutatividade: 


$$
\begin{aligned}
& {\left[\alpha_{m}, \alpha_{n}\right]=m \cdot \delta_{m,-n} K} \\
& {\left[K, \alpha_{m}\right]=0}
\end{aligned}
$$

Definindo $\alpha(z)=\sum_{n \in \mathbb{Z}} \alpha_{n} z^{-n-1}$, tem-se:

$$
[\alpha(z), \alpha(w)]=\partial_{w} \delta(z-w) K
$$

De fato,

$$
\begin{aligned}
{[\alpha(z), \alpha(w)] } & =\left[\sum_{n \in \mathbb{Z}} \alpha_{N} z^{-n-1}, \sum_{m \in \mathbb{Z}} \alpha_{m} w^{-m-1}\right] \\
& \stackrel{(2.24)}{=} \sum_{n \in \mathbb{Z}} n \cdot z^{-n-1} w^{n-1} K \\
& =\partial_{w}\left(\sum_{n \in \mathbb{Z}} z^{-n-1} w^{n}\right) K \\
& =\partial_{w} \delta(z-w) K
\end{aligned}
$$

Em outras palavras, a distribuição formal $\alpha(z)$ é local (com relação a ela mesma) com a seguinte $O P E$ considerada na álgebra envolvente universal de $S$ :

$$
\alpha(z) \alpha(w) \sim \frac{K}{(z-w)^{2}}
$$

A distribuição formal par $\alpha(z)$ é usualmente chamada de "free boson". Agora será introduzida a seguinte distribuição formal com valores em $\hat{\mathfrak{g}}$ que são usualmente chamados de correntes:

$$
a(z)=\sum_{n \in \mathbb{Z}} a_{n} z^{-n-1}
$$

onde $a \in \mathfrak{g}$. Então pela equivalência de $(b)$ e $(e)$ do Teorema 2.8, temos que:

$$
[a(z), b(w)]=\delta(z-w)[a, b](w)+\partial_{w} \delta(z-w)(a \mid b) K
$$


De fato, tem-se que:

$$
\begin{aligned}
{[a(z), b(w)] } & =\left[\sum_{n \in \mathbb{Z}} a_{n} z^{-n-1}, \sum_{m \in \mathbb{Z}} b_{m} z^{-m-1}\right] \\
& =\sum_{m, n \in \mathbb{Z}}\left[a_{n}, b_{m}\right] z^{-n-1} w^{-m-1} \\
& =\sum_{m, n \in \mathbb{Z}}[a, b]_{m+n} z^{-n-1} w^{-m-1}+\sum_{m \in \mathbb{Z}} m \cdot(a \mid b) z^{-m-1} w^{-n-1} K \\
& =\sum_{n \in \mathbb{Z}} z^{-n-1} w^{n} \cdot \sum_{m \in \mathbb{Z}}[a, b]_{m} w^{-m-1}+\partial_{w}\left(\sum_{m \in \mathbb{Z}} z^{-m-1} w^{m}\right)(a \mid b) K \\
& =\delta(z-w)[a, b](w)+\partial_{w} \delta(z-w)(a \mid b) K
\end{aligned}
$$

Desta forma, todas as correntes $a(z)$ são mutuamente locais com a seguinte $O P E$ na álgebra envolvente universal $U(\hat{\mathfrak{g}})$ de $\hat{\mathfrak{g}}$ :

$$
a(z) b(w) \sim \frac{[a, b](w)}{z-w}+\frac{(a \mid b) K}{(z-w)^{2}}
$$

Agora, para $a \in \mathfrak{g}$, define-se as supercorrentes:

$$
\bar{a}(z)=\sum_{n \in \mathbb{Z}} a_{n+\frac{1}{2}} z^{-n-1}
$$

onde $a_{n+\frac{1}{2}}=a \otimes t^{n} \theta$. Então as supercorrentes $\bar{a}(z)$ são mutuamente locais, e também são locais com respeito às correntes, com suas respectivas $O P E$ 's dadas por:

$$
\begin{aligned}
a(z) \bar{b}(w) & \sim \frac{\overline{[a, b]}(w)}{z-w} \\
\bar{a}(z) \bar{b}(w) & \sim \frac{(b \mid a) K}{z-w}
\end{aligned}
$$

De fato, valem (2.31) e (2.32) pois: 


$$
\begin{aligned}
{[a(z), \bar{b}(w)] } & =\left[\sum_{n \in \mathbb{Z}} a_{n} z^{-n-1}, \sum_{m \in \mathbb{Z}} b_{m+\frac{1}{2}} w^{-m-1}\right] \\
& =\sum_{m, n \in \mathbb{Z}}\left[a_{n}, b_{m+\frac{1}{2}}\right] z^{-n-1} w^{-m-1} \\
& =\sum_{m, n \in \mathbb{Z}}[a, b] \otimes t^{m+n} \theta w^{-m-n-1} z^{-n-1} w^{n} \\
& =\sum_{m, n \in \mathbb{Z}}[a, b] \otimes t^{m} \theta w^{-m-1} z^{-n-1} w^{n} \\
& =\sum_{n \in \mathbb{Z}} z^{-n-1} w^{n} \cdot \sum_{m \in \mathbb{Z}}[a, b] t^{m} \theta w^{-m-1} \\
& =\delta(z-w) \overline{[a, b]}(w)
\end{aligned}
$$

Agora (2.32) segue analogamente. As supercorrentes formam uma subálgebra fechada (sobre as $O P E$ 's). Em vista da sua importância, será repetida sua construção de forma ligeiramente diferente:

Seja $A$ um superespaço com forma bilinear anti-supersimétrica (isto é, $\left.(\varphi \mid \psi)=-(-1)^{p(\varphi)}(\psi \mid \varphi)\right)$. O afinizador de Clifford de $(A,(\cdot \mid \cdot))$ é a superálgebra de Lie

$$
C_{A}=A\left[t, t^{-1}\right]+\mathbb{C} K
$$

com relação de comutação $\left(m, n \in \frac{1}{2}+\mathbb{Z} ; \varphi, \psi \in A\right)$ :

$$
\begin{aligned}
& {\left[\varphi_{m}, \psi_{n}\right]=(\varphi \mid \psi) \delta_{m,-n} K} \\
& {\left[C_{A}, K\right]=0}
\end{aligned}
$$

onde $\varphi_{m}=\varphi \otimes t^{m-\frac{1}{2}}$. As distribuições formais $\varphi(z)=\sum_{n \in \mathbb{Z}} \varphi_{n+\frac{1}{2}} z^{-n-1}$ são mutuamente locais com a $O P E$ (na álgebra envolvente universal de $C_{A}$ ):

$$
\varphi(z) \varphi(w) \sim \frac{(\varphi \mid \psi) K}{z-w}
$$

De fato: 


$$
\begin{aligned}
{[\varphi(z), \psi(w)] } & =\left[\sum_{n \in \mathbb{Z}} \varphi_{n+\frac{1}{2}} z^{-n-1}, \sum_{m \in \mathbb{Z}} \psi_{m+\frac{1}{2}} w^{-m-1}\right] \\
& =\sum_{m, n \in \mathbb{Z}}\left[\varphi_{n+\frac{1}{2}}, \psi_{m+\frac{1}{2}}\right] z^{-n-1} w^{-m-1} \\
& \stackrel{(2.33)}{=} \sum_{m, n \in \mathbb{Z}}(\varphi \mid \psi) \delta_{n+\frac{1}{2},-m-\frac{1}{2}} K z^{-n-1} w^{-m-1} \\
& =\sum_{m \in \mathbb{Z}}(\varphi \mid \psi) K z^{-(-m-1)-1} w^{-m-1} \\
& =(\varphi \mid \psi) K \sum_{m \in \mathbb{Z}} z^{m} w^{-m-1} \\
& =\delta(z-w)(\varphi \mid \psi) K
\end{aligned}
$$

Agora dois casos importantes do afinizador Clifford são os seguintes:

(1) Seja $A$ um superespaço $C_{\varphi}$ ímpar unidimensional com forma bilinear $(\varphi \mid \varphi)=1$ e seja $K=1$. Então $C_{A}$ gera a álgebra:

$$
\varphi_{m} \varphi_{n}+\varphi_{n} \varphi_{m}=\delta_{m-n}
$$

$\operatorname{com} m, n \in \mathbb{Z}$, pois:

$$
\begin{aligned}
\varphi_{m} \varphi_{n}+\varphi_{n} \varphi_{m} & =\left[\varphi_{m}, \varphi_{n}\right] \\
& =(\varphi \mid \varphi) \delta_{m,-n} K \\
& =\delta_{m,-n}
\end{aligned}
$$

"neutral free fermion". Sua $O P E$ é dada por:

A distribuição formal (ímpar) $\varphi(z)=\sum_{n \in \frac{1}{2}+\mathbb{Z}} \varphi_{n} z^{-n-\frac{1}{2}}$ é conhecida como

$$
\varphi(z) \varphi(w) \sim \frac{1}{z-w}
$$

De fato: 


$$
\begin{aligned}
{[\varphi(z), \varphi(w)] } & =\left[\sum_{n \in \mathbb{Z}} \varphi_{n} z^{-n-\frac{1}{2}}, \sum_{m \in \frac{1}{2}+\mathbb{Z}} \varphi_{m} w^{-m-\frac{1}{2}}\right] \\
& =\sum_{m, n \in \frac{1}{2}+\mathbb{Z}}\left[\varphi_{n}, \varphi_{m}\right] z^{-n-\frac{1}{2}} w^{-m-\frac{1}{2}} \\
& =\sum_{m, n \in \frac{1}{2}+\mathbb{Z}} \delta_{m,-n} z^{-n-\frac{1}{2}} w^{-m-\frac{1}{2}} \\
& =\sum_{m, n \in \mathbb{Z}} z^{-\left(n+\frac{1}{2}\right)-\frac{1}{2}} w^{\left(n+\frac{1}{2}\right)-\frac{1}{2}} \\
& =\sum_{n \in \mathbb{Z}} z^{-n-1} w^{n} \\
& =\delta(w-z)
\end{aligned}
$$

(2) Seja $A$ um superespaço ímpar bidimensional $\mathbb{C} \varphi^{+} \oplus \mathbb{C} \varphi^{-}$, com forma bilinear simétrica $\left(\varphi^{+} \mid \varphi^{-}\right)=1$ e $\left(\varphi^{ \pm} \mid \varphi^{ \pm}\right)=0$ e ainda seja $K=1$. Então obtem-se a álgebra $\left(m, n \in \frac{1}{2}+\mathbb{Z}\right)$ :

$$
\begin{aligned}
& \varphi_{m}^{ \pm} \varphi_{n}^{ \pm}+\varphi_{n}^{ \pm} \varphi_{m}^{ \pm}=0 \\
& \varphi_{m}^{ \pm} \varphi_{n}^{\mp}+\varphi_{n}^{\mp} \varphi_{m}^{ \pm}=\delta_{m,-n}
\end{aligned}
$$

de forma análoga à (2.35). A distribuição formal ímpar $\varphi^{ \pm}(z)=$ $\sum_{n \in \frac{1}{2}+\mathbb{Z}} \varphi_{n}^{ \pm} z^{-n-\frac{1}{2}}$ é chamada de "charged free fermions". Sua $O P E$ é:

$$
\begin{aligned}
\varphi^{ \pm}(z) \varphi^{\mp}(w) & \sim \frac{1}{z-w} \\
\varphi^{ \pm}(z) \varphi^{ \pm}(w) & \sim 0
\end{aligned}
$$

cuja demonstração é análoga à de (2.36).

\subsection{Pesos Conformes}

Seja $H: U \rightarrow U$ uma derivação diagonalizável da álgebra associativa $U$, chamada Hamiltoniana. Então $H$ age no espaço de todas as distribuições formais com valores em $U$ de forma natural (coeficiente à coeficiente), isto é,

$$
H\left(a_{n} \cdot z^{-n-1}\right)=H\left(a_{n}\right) \cdot z^{-n-1}
$$

Assim, tem-se: 
Definição 2.25. Diz-se que $a\left(z_{1}, \cdots, z_{m}\right)$ é uma auto-distribuição de $H$ com peso conforme $\Delta \in \mathbb{C}$ se:

$$
\left(H-\Delta-\sum_{i=1}^{n} z_{i} \partial_{z_{i}}\right) a=0
$$

Desta forma, tem-se:

Proposição 2.26. Suponha que $a$ e $b$ são auto-distribuições de pesos conformes $\Delta_{1}$ e $\Delta_{2}$, respectivamente. Então:

(a) $\partial_{z_{i}} a$ é uma auto-distribuição de peso conforme $\Delta_{1}+1$, para todo $i \in\{1, \ldots m\}$.

(b) : a $(z) b(w)$ : é uma auto-distribuição de peso conforme $\Delta_{1}+\Delta_{2}$.

(c) O n-ésimo coeficiente da OPE de $[a(z), b(w)]$ é uma auto-distribuição de peso conforme $\Delta_{1}+\Delta_{2}-n-1$, onde $n \in \mathbb{Z}_{+}$.

(d) Se $f\left(=f\left(z_{1}, \cdots, z_{m}\right)\right)$ é uma função homogênea de grau $j$, então fa é uma auto-distribuição de peso conforme $\Delta-j$.

Demonstração. Para provar (a), basta observar que:

$$
\begin{aligned}
& \left(H-\Delta-\sum_{i=1}^{m} z_{i} \partial_{z_{i}}\right) a=0 \Rightarrow H a-\Delta a-z_{1} \partial_{z_{1}} a-\ldots-z_{m} \partial_{z_{m}} a=0 \\
& \Rightarrow \partial_{z_{1}}\left(H a-\Delta a-z_{1} \partial_{z_{1}} a-\ldots-z_{m} \partial_{z_{m}} a\right)=0 \\
& \Rightarrow H \partial_{z_{1}} a-\Delta \partial_{z_{1}} a-\partial_{z_{1}} a-z_{1} \partial_{z_{1}}^{2} a-\sum_{i=2}^{m} z_{i} \partial_{z_{i}} \partial_{z_{1}} a=0 \\
& \Rightarrow\left(H-(\Delta+1)-\sum_{i=1}^{m} z_{i} \partial_{z_{i}}\right) \partial_{z_{1}} a=0
\end{aligned}
$$

Logo $\partial_{z_{i}} a$ é uma auto-distribuição de peso conforme $\Delta+1$. Agora, provemos $(b)$ :

$$
\begin{aligned}
& \left(H-\Delta_{1}-\Delta_{2}-z \partial_{z}-w \partial_{w}\right) a(z) b(w)=H a(z) b(w)+a(z) H b(w) \\
& -\Delta_{1} a(z) b(w)-\Delta_{2} a(z) b(w)-z \partial_{z} a(z) b(w)-w \partial_{w} a(z) b(w) \\
& =0 \cdot b(w)+a(z) \cdot 0=0
\end{aligned}
$$

Agora:

$$
: a(z) b(w):=a(z)_{+} b(w)+(-1)^{p(a) p(b)} b(w) a(z)_{-}
$$


logo é suficiente provar que $a(z)_{+}$e $a(z)_{-}$possuem o mesmo peso conforme $\Delta_{1}$ de $a(z)$ para que (b) fique provado, uma vez que a soma de duas autodistribuições de mesmo peso conforme $\Delta$ é também uma auto-distribuição de peso conforme $\Delta$. Assim:

$$
\begin{aligned}
\left(H-\Delta-z \partial_{z}\right) a(z) & =\left(H-\Delta-z \partial_{z}\right) \sum_{n \in \mathbb{Z}} a_{(n)} z^{-n-1} \\
& =\sum_{n \in \mathbb{Z}}\left(H\left(a_{(n)}\right)-\Delta a_{(n)}+(n+1) a_{(n)}\right) z^{-n-1} \\
& =0
\end{aligned}
$$

Logo:

$$
H\left(a_{(n)}\right)-\Delta a_{(n)}+(n+1) a_{(n)}=0
$$

para todo $n \in \mathbb{Z}$. Em particular, isto vale para $n \in \mathbb{Z}_{+}^{*}$. Logo

$$
\left(H-\Delta-z \partial_{z}\right) a(z)_{-}=0
$$

Analogamente, segue que $\left(H-\Delta-z \partial_{z}\right) a(z)_{+}=0$. Assim, está provado (b). Quanto ao ítem $(c)$, sabemos que $[a(z), b(w)]$ tem peso conforme $\Delta_{1}+\Delta_{2}$ (pois $\left.[a(z), b(w)]=a(z) b(w)-(-1)^{p(a) p(b)} b(w) a(z)\right)$. Agora sabe-se que

$$
a(w)_{(n)} b(w)=\operatorname{Res}_{z}[a(z), b(w)](z-w)^{n}
$$

Pelo ítem $(d)$ a ser provado abaixo, tem-se que $[a(z), b(w)](z-w)^{n}$ tem peso conforme $\Delta_{1}+\Delta_{2}-n$. Assim, basta mostrar que o operador $R_{e}$ faz o peso conforme de uma auto-distribuição ser reduzido em 1 unidade. Sendo assim, seja

$$
u(z, w)=\sum_{m, n \in \mathbb{Z}} a_{m, n} z^{m} w^{n}
$$

uma auto-distribuição de peso conforme $N$. Mostremos que 


$$
\operatorname{Res}_{z} u(z, w)=\sum_{n \in \mathbb{Z}} a_{-1, n} w^{n}
$$

tem peso conforme $N-1$. Sabemos que $H: U \rightarrow U$ é uma derivação diagonalizável. Então:

$$
\begin{aligned}
& H(a b)=H(a) b+a H(b) \\
& H u(z, w)=\sum_{m, n \in \mathbb{Z}} H\left(a_{m, n}\right) z^{m} w^{n}
\end{aligned}
$$

Agora:

$$
\begin{aligned}
N u(z, w) & =\left(H-z \partial_{z}-w \partial_{w}\right) u(z, w) \\
& =\sum_{m, n \in \mathbb{Z}}\left(H\left(a_{m, n}\right)-(m+n) a_{m, n}\right) z^{m} w^{n}
\end{aligned}
$$

Logo:

$N a_{m, n}=H\left(a_{m, n}\right)-(m+n) a_{m, n}$

para todo $m, n \in \mathbb{Z}$. Portanto

$H\left(a_{m, n}\right)=(N+m+n) a_{m, n} \Rightarrow H\left(a_{-1, n}\right)=(N+n-1) a_{-1, n}$

Assim, temos que:

$$
\begin{aligned}
\left(H-w \partial_{w}\right) \operatorname{Res}_{z} u(z, w) & =\left(H-w \partial_{w}\right) \sum_{n \in \mathbb{Z}} a_{-1, n} w^{n} \\
& =\sum_{n \in \mathbb{Z}}\left(H\left(a_{-1, n}\right)-n \cdot a_{-1, n}\right) w^{n} \\
& =\sum_{n \in \mathbb{Z}}(N+n-1-n) a_{-1, n} w^{n} \\
& =(N-1) \sum_{n \in \mathbb{Z}} a_{-1, n} w^{n} \\
& =(N-1) \operatorname{Res}_{z} u(z, w)
\end{aligned}
$$

Logo o peso conforme de $\operatorname{Res}_{z} u(z, w)$ é, de fato, igual a $N-1$, o que prova $(c)$. Finalmente, provemos $(d)$. Temos:

$$
f\left(t z_{1}, \ldots, t z_{m}\right)=t^{j} f\left(z_{1}, \ldots, z_{m}\right)
$$


Derivando em $t$, obtemos:

$$
\sum_{i=1}^{m} z_{i} \cdot \partial_{z_{i}} f\left(t z_{1}, \ldots, t z_{m}\right)=j \cdot t^{j-1} f\left(z_{1}, \ldots, z_{m}\right)
$$

Fazendo $t=1$, temos:

$$
\sum_{i=1}^{m} z_{i} \cdot \partial_{z_{i}} f\left(z_{1}, \ldots, z_{m}\right)=j \cdot f\left(z_{1}, \ldots, z_{m}\right)
$$

Portanto:

$$
\begin{aligned}
& \left(H-\Delta+j-z_{1} \partial_{z_{1}}-z_{2} \partial_{z_{2}}-\ldots-z_{m} \partial_{z_{m}}\right) f a= \\
& =H(f a)-\Delta f a+j \cdot f a-z_{1} \partial_{z_{1}}(f a)-z_{2} \partial_{z_{2}}(f a)-\ldots-z_{m} \partial_{z_{m}}(f a) \\
& =f H(a)-f \Delta a+j \cdot f a-\left[\left(z_{1} \partial_{z_{1}}+z_{2} \partial_{z_{2}}+\ldots+z_{m} \partial_{z_{m}}\right) f a\right. \\
& \left.+f\left(z_{1} \partial_{z_{1}}+z_{2} \partial_{z_{2}}+\ldots+z_{m} \partial_{z_{m}}\right) a\right] \\
& =f H(a)-f \Delta a+j \cdot f a-j \cdot f a-f\left(z_{1} \partial_{z_{1}}+z_{2} \partial_{z_{2}}+\ldots+z_{m} \partial_{z_{m}}\right) a \\
& =f H(a)-f \Delta a-f\left(z_{1} \partial_{z_{1}}+z_{2} \partial_{z_{2}}+\ldots+z_{m} \partial_{z_{m}}\right) a \\
& =f \cdot\left(H-\Delta-z_{1} \partial_{z_{1}}-z_{2} \partial_{z_{2}}-\ldots-z_{m} \partial_{z_{m}}\right) a \\
& =f \cdot 0 \\
& =0
\end{aligned}
$$

o que prova $(d)$.

Corolário 2.27. Se a $(z)$ e b(z) são auto-distribuições mutuamente locais de pesos conformes $\Delta_{1}$ e $\Delta_{2}$, então na OPE:

$$
a(z) b(w) \sim \sum_{j=0}^{N-1} \frac{c^{j}(w)}{(z-w)^{j+1}}
$$

todos os somandos possuem o mesmo peso conforme $\Delta_{1}+\Delta_{2}$.

Demonstração. Sabemos que $c^{j}(w)=\operatorname{Res}_{z} a(z) b(w)(z-w)^{j}$. Também sabemos que $a(z) b(w)$ possui peso conforme $\Delta_{1}+\Delta_{2}$ e como $(z-w)^{j}$ é homogêneo de grau $j$, temos que $a(z) b(w)(z-w)^{j}$ possui peso conforme $\Delta_{1}+\Delta_{2}-j$. Desta forma, $\operatorname{Res}_{z} a(z) b(w)(z-w)^{j}$ possui peso conforme $\Delta_{1}+\Delta_{2}-j-1$. Além disso: 
$i_{z, w} \frac{1}{(z-w)^{j+1}}=\sum_{m=0}^{\infty}\left(\begin{array}{c}m \\ j\end{array}\right) z^{-m-1} w^{m-j}$

que é, portanto, homogêneo de grau $-j-1 . \quad$ Logo $c^{j}(w) i_{z, w} \frac{1}{(z-w)^{j+1}}$ possui peso conforme $\Delta_{1}+\Delta_{2}$.

Caso $a(z)$ seja uma auto-distribuição de peso conforme $\Delta$, usualmente a escreveremos na forma:

$a(z)=\sum_{n \in-\Delta+\mathbb{Z}} a_{n} z^{-n-\Delta}$

A condição de $a(z)$ ser uma auto-distribuição de peso conforme $\Delta$ é equivalente a:

$$
H a_{n}=-n \cdot a_{n}
$$

De fato:

$\left(H-\Delta-z \partial_{z}\right) a(z)=0$

se, e somente se,

$H a(z)-\Delta a(z)-z \partial_{z} a(z)=0$

se, e somente se,

$H\left(\sum_{n \in-\Delta+\mathbb{Z}} a_{n} z^{-n-\Delta}\right)-\Delta\left(\sum_{n \in-\Delta+\mathbb{Z}} a_{n} z^{-n-\Delta}\right)-z \partial_{z}\left(\sum_{n \in-\Delta+\mathbb{Z}} a_{n} z^{-n-\Delta}\right)=0$

se, e somente se,

$\sum_{n \in-\Delta+\mathbb{Z}} H\left(a_{n} z^{-n-\Delta}\right)+\sum_{n \in-\Delta+\mathbb{Z}}\left(-\Delta a_{n}\right) z^{-n-\Delta}+\sum_{n \in-\Delta+\mathbb{Z}}(n+\Delta) a_{n} z^{-n-\Delta}=0$

se, e somente se,

$\sum_{n \in-\Delta+\mathbb{Z}} H\left(a_{n}\right) z^{-n-\Delta}=\sum_{n \in-\Delta+\mathbb{Z}}\left(-n \cdot a_{n}\right) z^{-n-\Delta}$ 
Logo $H\left(a_{n}\right)=-n \cdot a_{n}$ se, e somente se, $a(z)$ tem peso conforme $\Delta$. Analogamente ao Teorema $2.8(e)$, temos:

$$
\begin{gathered}
{\left[a_{m}, b_{n}\right]=\sum_{j=0}^{N-1}\left(\begin{array}{c}
m+\Delta-1 \\
j
\end{array}\right) c_{m+n}^{j}} \\
{\left[a_{m}, b(z)\right]=\sum_{j=0}^{N-1}\left(\begin{array}{c}
m+\Delta-1 \\
j
\end{array}\right) c^{j}(z) z^{m+\Delta-j-1}}
\end{gathered}
$$

Exemplo 2.28. Escolhendo para a álgebra corrente $\hat{\mathfrak{g}}$ o Hamiltoniano $H=-t \partial_{t}$ e para a supercorrente $\hat{\mathfrak{g}}_{\text {super }}$ o Hamiltoniano $H=-t \partial_{t}-\frac{1}{2} \theta \partial_{\theta}$, então temos que as correntes a(z) tem peso conforme 1 enquanto que as supercorrentes $\bar{a}(z)$ tem peso conforme $\frac{1}{2}$. De fato:

$$
\begin{aligned}
\left(H-\Delta-z \partial_{z}\right) a(z)=0 & \Rightarrow\left(H-\Delta-z \partial_{z}\right)\left(\sum_{n \in \mathbb{Z}} a_{n} z^{-n-1}\right)=0 \\
& \Rightarrow \sum_{n \in \mathbb{Z}}\left(H\left(a_{n}\right)-\Delta a_{n}+(n+1) a_{n}\right) z^{-n-1}=0 \\
& \Rightarrow H\left(a_{n}\right)-\Delta a_{n}+(n+1) a_{n}=0 \\
& \Rightarrow-n a_{n}-\Delta a_{n}+n a_{n}+a_{n}=0 \\
& \Rightarrow \Delta=1
\end{aligned}
$$

Da mesma maneira:

$$
\begin{aligned}
\left(H-\Delta-z \partial_{z}\right) \bar{a}(z)=0 & \Rightarrow\left(H-\Delta-z \partial_{z}\right)\left(\sum_{n \in \mathbb{Z}} a_{n+\frac{1}{2}} z^{-n-1}\right)=0 \\
& \Rightarrow \sum_{n \in \mathbb{Z}}\left(H\left(a_{n+\frac{1}{2}}\right)-\Delta a_{n+\frac{1}{2}}+(n+1) a_{n+\frac{1}{2}}\right) z^{-n-1}=0 \\
& \Rightarrow H\left(a_{n+\frac{1}{2}}\right)-\Delta a_{n+\frac{1}{2}}+(n+1) a_{n+\frac{1}{2}}=0 \\
& \Rightarrow\left(-n-\frac{1}{2}\right) a_{n+\frac{1}{2}}-(n+1-\Delta) a_{n+\frac{1}{2}}=0 \\
& \Rightarrow \Delta=\frac{1}{2}
\end{aligned}
$$

onde $H=-t \partial_{t} e ́$, de fato, um Hamiltoniano já que: 


$$
\begin{aligned}
H\left(a_{n}\right) & =H\left(a \otimes t^{n}\right) \\
& =-t \partial_{t}\left(a \otimes t^{n}\right) \\
& =-t\left(n a \otimes t^{n-1}\right) \\
& =-n a \otimes t^{n} \\
& =-n a_{n}
\end{aligned}
$$

Analogamente, considerando $H=-t \partial_{t}-\frac{1}{2} \theta \partial_{\theta}$, pode-se calcular o peso conforme de $\bar{a}(z)$.

O Corolário 2.27 fornece um resultado muito útil para o cálculo da $O P E$. Em muitos exemplos (por exemplo, nas considerações de unitariedade), o peso conforme está em $\frac{1}{2} \mathbb{Z}_{+}$, sendo igual à zero se e somente se a auto-distribuição é um elemento constante comutando com as distribuições formais da teoria. Com a condição de positividade acima, segue do Corolário 2.27 que duas auto-distribuições mutuamente locais de peso conforme $\frac{1}{2}$ tem a $O P E$ da forma (2.32). Se ambas tiverem peso conforme 1, então a $O P E$ será da forma (2.29) e caso uma tenha peso 1 e a outra tenha peso conforme $\frac{1}{2}$, então a $O P E$ será dada por (2.31). Agora será apresentada uma autodistribuição par $L(z)$ local (com ela mesmo) de peso conforme 2. Seja:

$$
L(z)=\sum_{n \in \mathbb{Z}} L_{n} z^{-n-2}
$$

Assim, sua $O P E$ tem a forma

$$
L(z) L(w) \sim \frac{\frac{1}{2} c}{(z-w)^{4}}+\frac{a(w)}{(z-w)^{3}}+\frac{2 b(w)}{(z-w)^{2}}+\frac{c(w)}{z-w}
$$

onde c é uma distribuição formal constante. Isto ocorre porque o Corolário 2.27 diz que todos os somandos tem peso conforme $\Delta_{1}+\Delta_{2}=2+2=4$. Agora $c$ é constante, pois caso contrário, seu peso conforme seria diferente de 0 e como

$$
i_{z, w} \frac{1}{(z-w)^{4}}
$$

tem peso conforme 4 , então seu produto não teria peso conforme 4 . 
Teorema 2.29. Suponha que $L(z)$ é uma distribuição formal local par com a OPE da forma (2.43). Então:

(a) $a(w)=0$

$$
c(w)=\partial b(w)
$$

(b) Se, além disso, $[c, L(z)]=0 e$

$$
\begin{aligned}
& {\left[L_{-1}, L(z)\right]=\partial(z) ;} \\
& {\left[L_{0}, L(z)\right]\left(z \partial_{z}+2\right) L(z)}
\end{aligned}
$$

então (2.43) fica:

$$
L(z) L(w) \sim \frac{\frac{1}{2} c}{(z-w)^{4}}+\frac{2 L(w)}{(z-w)^{2}}+\frac{\partial L(w)}{z-w}
$$

ou equivalentemente, tem-se a álgebra de Virasoro $(m, n \in \mathbb{Z})$ :

$$
\begin{aligned}
& {\left[L_{m}, L_{n}\right]=(m-n) L_{m+n}+\frac{m^{3}-m}{12} \delta_{m,-n} c} \\
& {\left[c, L_{m}\right]=0}
\end{aligned}
$$

Demonstração. Por (2.43), temos que:

$$
L(z) L(w) \sim \frac{\frac{1}{2} c}{(z-w)^{4}}+\frac{a(w)}{(z-w)^{3}}+\frac{2 b(w)}{(z-w)^{2}}+\frac{c(w)}{z-w}
$$

Logo:

$$
L(w) L(z) \sim \frac{\frac{1}{2} c}{(z-w)^{4}}-\frac{a(z)}{(z-w)^{3}}+\frac{2 b(z)}{(z-w)^{2}}-\frac{c(z)}{z-w}
$$

Pela fórmula de Taylor, temos que:

$$
\begin{aligned}
L(w) L(z) & \sim \frac{\frac{1}{2} c}{(z-w)^{4}}-\frac{a(w)+\partial a(w)(z-w)+\partial^{(2)} a(w)(z-w)^{2}}{(z-w)^{3}} \\
& +\frac{2 b(w)+2 \partial b(w)(z-w)}{(z-w)^{2}}-\frac{c(w)}{z-w}
\end{aligned}
$$


Logo:

$$
\begin{aligned}
L(w) L(z) & \sim \frac{\frac{1}{2} c}{(z-w)^{4}}-\frac{a(w)}{(z-w)^{3}}+\frac{2 b(w)+\partial a(w)}{(z-w)^{2}} \\
& +\frac{\partial^{(2)} a(w)+\partial b(w)-c(w)}{z-w}
\end{aligned}
$$

Logo:

$a(w)=-a(w)$

$2 b(w)=2 b(w)+\partial a(w)$

$c(w)=\partial^{(2)} a(w)+2 \partial b(w)-c(w)$

e, desta forma, temos que $a(w)=0$ e que $c(w)=\partial b(w)$. Logo, temos:

$$
L(z) L(w) \sim \frac{\frac{1}{2} c}{(z-w)^{4}}+\frac{2 b(w)}{(z-w)^{2}}+\frac{\partial b(w)}{z-w}
$$

Por (2.42), temos que:

$\left[L_{-1}, L(z)\right]=\partial b(z)$

$\left[L_{0}, L(z)\right]=\left(z \partial_{z}+2\right) b(z)$

De fato, (2.42) nos diz que:

$\left[a_{m}, b(z)\right]=\sum_{j=0}^{N-1}\left(\begin{array}{c}m+\Delta-1 \\ j\end{array}\right) c^{j}(z) z^{m+\Delta-j-1}$

Logo:

$$
\begin{aligned}
{\left[L_{-1}, L(z)\right] } & =\sum_{j=0}^{N-1}\left(\begin{array}{c}
-1+2-1 \\
j
\end{array}\right) c^{j}(z) z^{-j} \\
& =c^{0}(z)
\end{aligned}
$$




$$
\begin{aligned}
{\left[L_{0}, L(z)\right] } & =\sum_{j=0}^{N-1}\left(\begin{array}{c}
0+2-1 \\
j
\end{array}\right) c^{j}(z) z^{1-j} \\
& =c^{0}(z) \cdot z+c^{1}(z)
\end{aligned}
$$

Agora o Corolário 2.27 nos diz que:

$$
a(z) b(w) \sim \sum_{j=0}^{N-1} \frac{c^{j}(w)}{(z-w)^{j+1}}
$$

Logo $c^{0}(z)$ é o coeficiente de $i_{z, w} \frac{1}{z-w}$ e $c^{1}(z)$ é o de $i_{z, w} \frac{1}{(z-w)^{2}}$. Desta forma:

$$
\begin{aligned}
{\left[L_{-1}, L(z)\right] } & =c^{0}(z) \\
& =\partial b(z)
\end{aligned}
$$

e também

$$
\begin{aligned}
{\left[L_{0}, L(z)\right] } & =z \cdot \partial_{z} b(z)+2 b(z) \\
& =\left(z \partial_{z}+2\right) b(z)
\end{aligned}
$$

Assim, por (2.44), temos que $L(z)=b(z)$, o que prova (2.45). Já a equação (2.46) é equivalente a esta $O P E$ por (2.41). De fato, temos:

$$
\begin{aligned}
{\left[L_{m}, L_{n}\right] } & =\sum_{j=0}^{N-1}\left(\begin{array}{c}
m+2-1 \\
j
\end{array}\right) c_{m+n}^{j} \\
& =\left(\begin{array}{c}
m+1 \\
0
\end{array}\right) c_{m+n}^{0}+\left(\begin{array}{c}
m+1 \\
1
\end{array}\right) c_{m+n}^{1}+\left(\begin{array}{c}
m+1 \\
3
\end{array}\right) c_{m+n}^{3}
\end{aligned}
$$

Agora $c^{j}(w)=\sum_{k \in \mathbb{Z}} c_{k}^{j} w^{j-k-3}$ pois como já sabemos, $c_{m+n}^{j}$ é o coeficiente de $w^{-(m+\Delta-1)-(n+\Delta-1)+j-1}$. De $L(w)=\sum_{n \in \mathbb{Z}} L_{n} w^{-n-2}$, segue que:

$$
\partial L(w)=\sum_{n \in \mathbb{Z}}(-n-2) L_{n} w^{-n-3}
$$

Assim: 


$$
c^{0}(w)=\partial L(w) \Rightarrow c_{m+n}^{0}=(-n-m-2) L_{m+n}
$$

já que $c_{m+n}^{0}$ é o coeficiente de $w^{-m-n-3}$ em $c^{0}(w)$ e o coeficiente em $\partial L(w)$ é $(-m-n-2) \cdot L_{m+n}$. Agora:

$$
c^{1}(w)=2 L(w) \Rightarrow c_{m+n}^{1}=2 L_{m+n}
$$

já que $c_{m+n}^{1}$ é o coeficiente de $w^{-m-n-2}$ em $c^{1}(w)$ e $2 L_{m+n}$ é o coeficiente de $w^{-m-n-2}$ em $2 L(w)$. Finalmente:

$$
c^{3}(w)=\frac{1}{2} c \Rightarrow c_{m+n}^{3}=\frac{1}{2} c \delta_{m,-n}
$$

já que $c_{m+n}^{3}$ é o coeficiente de $w^{-m-n}$ em $c^{3}(w)$, que é $\frac{1}{2} c$ se $-m-n=0$, isto é, se $m=-n$, e será 0 caso contrário. Portanto:

$$
\begin{aligned}
{\left[L_{m}, L_{n}\right] } & =1 \cdot c_{m+n}^{0}+(m+1) \cdot L_{m+n}^{1}+\frac{m^{3}-m}{6} c_{m+n}^{3} \\
& =(-n-m-2) \cdot L_{m+n}+(2 m+2) \cdot L_{m+n}+\frac{m^{3}-m}{12} c \delta_{m,-n} \\
& =(m-n) \cdot L_{m+n}+\frac{m^{3}-m}{12} \delta_{m,-n} c
\end{aligned}
$$

Agora $0=[c, L(z)]=\sum_{m \in \mathbb{Z}}\left[c, L_{m}\right] z^{-m-2}$ e, portanto $\left[c, L_{m}\right]=0, \forall m$. 


\section{Capítulo 3}

\section{Superálgebras Conformes}

\subsection{Introdução}

Neste Capítulo, define-se o conceito de superálgebra conforme (ou conformal) e o associa às superálgebras de Lie de distribuições formais através da teoria de Categorias. Depois introduz-se a noção de $\lambda$-colchete que será utilizada no Corolário 3.9 com o objetivo de concluir que uma álgebra Conforme finita é soma direta de uma subálgebra central de dimensão finita com um $\mathbb{C}[\partial]$-módulo livre de posto finito. Depois disso, constrói-se uma superálgebra de Lie de distribuições formais associada à álgebra conforme $R$, a qual denotaremos por LieR. Da mesma forma, define-se o conformalizador (Con) que faz a associação contrária.

$\mathrm{O}$ que temos (pelo Teorema 3.20) é que ConLieR $\simeq R$, mas não vale que $\operatorname{LieCon}(\mathfrak{g}, F) \simeq(\mathfrak{g}, F)$. Entretanto (pelo Teorema 3.21), temos que $\operatorname{LieCon}(\mathfrak{g}, F) \simeq(\mathfrak{g} / I, F / I)$, onde $I$ é um determinado ideal, que estabelece uma relação entre as superálgebras de Lie e as superálgebras de Lie de distribuições formais. Assim, basta estudar um e transportar o resultado para o outro (Cuidado: Con e Lie não são o inverso um do outro, já que não vale $\operatorname{Lie} \operatorname{Con}(\mathfrak{g}, F) \simeq(\mathfrak{g}, F)$, mas pelo menos já foi estabelecido um certo tipo de "equivalência" que permite tal associação).

A partir deste resultado, começa-se a analisar o problema da classificação apresentando três exemplos de superálgebras conformes finitas e finaliza-se o Capítulo enunciando um dos resultados de $[D K]$ que os utiliza. Sendo assim, tem-se com a seguinte definição:

Definição 3.1. Seja $\mathfrak{g}$ uma superálgebra de Lie arbitrária. Defina $f d(\mathfrak{g})$ como o espaço de todas as distribuições formais $\mathfrak{g}$-valuadas em $z$ munidas do $n$-ésimo produto da Definição $2.9(i)$. Este é um $\mathbb{C}[\partial]$-módulo, com $\partial=\partial_{z}$. 
Definição 3.2. Seja $R$ um subespaço sobre $\mathbb{C}$ de $f d(\mathfrak{g})$ que é fechado sobre todos os $n$-ésimos produtos, com $n \in \mathbb{Z}_{+}$. Defina $\mathfrak{g}(R)$ como sendo o $\mathbb{C}$ espaço de todos os coeficientes de todas as distribuiçôes formais sobre $R$.

Provado que todas as distribuições formais sobre $R$ são mutuamente locais, então $\mathfrak{g}(R)$ será uma subálgebra de $\mathfrak{g}$ com o colchete:

$$
\left[a_{(m)}, b_{(n)}\right]=\sum_{j \in \mathbb{Z}_{+}}\left(\begin{array}{c}
m \\
j
\end{array}\right)\left(a_{(j)} b\right)_{(m+n-j)}
$$

De fato,

$$
a(w)_{(n)} b(w)=\operatorname{Res}_{z}[a(z), b(w)](z-w)^{n}
$$

e também

$$
c^{n}(w)=\operatorname{Res}_{z} a(z, w)(z-w)^{n}
$$

Isto junto com o teorema 2.8(e) mostra que:

$$
\begin{array}{r}
{\left[a_{(m)}, b_{(n)}\right]=\sum_{j=0}^{N-1}\left(\begin{array}{c}
m \\
j
\end{array}\right) c_{(m+n-j)}^{j}} \\
\operatorname{Logo}\left[a_{(m)}, b_{(n)}\right]=\sum_{j \in \mathbb{Z}_{+}}\left(\begin{array}{c}
m \\
j
\end{array}\right)\left(a_{(j)} b\right)_{(m+n-j)}
\end{array}
$$

Claramente, $\mathfrak{g}(R)$ é uma superálgebra de Lie de distribuições formais.

Definição 3.3. Seja F uma coleção de distribuições formais mutuamente locais em $f d(\mathfrak{g})$. Defina o fecho de $F$, denotando-o por $\bar{F}$ como o $\mathbb{C}[\partial]$ módulo minimal de $f d(\mathfrak{g})$ que é fechado sobre todos os n-ésimos produtos (e que contém $F$ ), com $n \in \mathbb{Z}_{+}$.

Como será visto adiante, $\bar{F}$ consiste de distribuiçôes formais mutuamente locais e, desta forma, tem-se uma superálgebra de Lie de distribuições formais $\mathfrak{g}(\bar{F})$.

Definição 3.4. Uma superálgebra conforme $R$ é um $\mathbb{C}[\partial]$-módulo à esquerda, $\mathbb{Z} / 2 \mathbb{Z}$-graduado $R=R_{\overline{0}} \oplus R_{\overline{1}}$ munido de uma família de $\mathbb{C}$-produtos bilineares 
$\cdot(n) \cdot: R \times R \rightarrow R$

indexado por inteiros positivos, satisfazendo os seguintes axiomas, para todos os $a, b, c \in R$ e $m, n \geq 0$ :

$\left(C_{1}\right) a_{(n)} b=0$ para $n \gg 0$

$\left(C_{2}\right)(\partial a)_{(n)} b=-n \cdot a_{(n-1)} b$

$\left(C_{3}\right) a_{(n)} b=-(-1)^{p(a) p(b)} \sum_{j \geq 0}(-1)^{j+n} \partial^{(j)}\left(b_{(n+j)} a\right)$

$\left(C_{4}\right) a_{(m)}\left(b_{(n)} c\right)=\sum_{j \geq 0}\left(\begin{array}{c}m \\ j\end{array}\right)\left(a_{(j)} b\right)_{(m+n-j)} c+(-1)^{p(a) p(b)} b_{(n)}\left(a_{(m)} c\right)$

Os axiomas $\left(C_{1}\right)$ e $\left(C_{2}\right)$ implicam:

$\left(C_{2}\right)^{\prime} a_{(n)} \partial b=\partial\left(a_{(n)} b\right)+n \cdot a_{(n-1)} b$

e, desta forma, $\partial$ é uma derivação de todos os $n$-ésimos produtos.

Diz-se que uma superálgebra de Lie conforme sobre $\mathbb{C}$ é finita se for finitamente gerada como um $\mathbb{C}[\partial]$-módulo. Também diz-se que um elemento $a \in R$ é central se $a_{(n)} R=0$, para todo $n \in \mathbb{Z}_{+}\left(\operatorname{logo} R_{(n)} a=0\right.$ por $\left.\left(C_{3}\right)\right)$.

Teorema 3.5. São verdadeiras as seguintes afirmações:

(i) o operador $a_{(0)}$ é uma derivação.

(ii) $a_{(0)}$ e $\partial$ comutam

(iii) $\partial R$ é um ideal bilateral de $R$ (com respeito ao 0-ésimo produto).

(iv) $R / \partial R$ é uma superálgebra de Lie.

(v) A ação de $R / \partial R \times R \rightarrow R$ que o 0-ésimo produto induz em $R$ está bem-definida.

(vi) $R / \partial R$ comuta com $\mathbb{C}[\partial]$.

Demonstração. Por $\left(C_{4}\right)$, temos que 


$$
\begin{aligned}
a_{(0)}\left(b_{(n)} c\right) & =\sum_{j=0}^{0}\left(\begin{array}{l}
0 \\
j
\end{array}\right)\left(a_{(j)} b\right)_{(0+n-j)} c+(-1)^{p(a) p(b)} b_{(n)}\left(a_{(0)} c\right) \\
& =\left(a_{(0)} b\right)_{(n)} c+(-1)^{p(a) p(b)} b(n)\left(a_{(0)} c\right)
\end{aligned}
$$

Logo $a_{(0)}$ é uma derivação. Agora:

$$
\begin{aligned}
a_{(0)} \partial b & =\partial\left(a_{(0)} b\right)+0 \\
& =\partial\left(a_{(0)} b\right)
\end{aligned}
$$

e portanto, temos que $a_{(0)}$ e $\partial$ comutam. Claramente $0 \in \partial R$ e $a-b \in \partial R$ se $a, b \in \partial R$. Agora $(\partial R)_{(0)} R=0 \in \partial R$ pois $(\partial a)_{(0)} b=0$ pelo axioma $\left(C_{2}\right)$, e assim $\partial R$ é um ideal à direita. Agora é um ideal à esquerda já que $R_{(0)} \partial R \in \partial R$ pelo axioma $\left(C_{2}\right)^{\prime}$, o que prova que $\partial R$ é um ideal bilateral de $R$. Para mostrar que $R / \partial R$ é uma superálgebra de Lie, basta observar que $\left[r+\partial R, r_{1}+\partial R\right]=r_{(0)} r_{1}+\partial R$ é bilinear e:

(a) Vale a anti-comutatividade: De fato, $\left[r+\partial R, r_{1} \partial R\right]=0+\partial R$ pois (usando o axioma $\left.\left(C_{1}\right)\right)$ :

$$
\begin{aligned}
& r_{(0)} r=-(-1)^{j+0} \partial^{(j)}\left(r_{(j)} r\right)=-r_{(0)} r+\partial\left(-\sum_{j=1}^{\infty} \frac{(-1)^{j}}{j !} \partial_{j-1}\left(r_{(j)} r\right)\right) \\
& \text { Logo } r_{(0)} r=\partial\left(-\frac{1}{2} \sum_{j=1}^{\infty} \frac{(-1)^{j}}{j !} \partial^{j-1}\left(r_{(j)} r\right)\right) \in \partial R \text { e assim, temos que: } \\
& {[r+\partial R, r+\partial R]=r_{(0)} r+\partial R} \\
& =0+\partial R
\end{aligned}
$$

(2) Superidentidade de Jacobi: $\left[r+\partial R,\left[r^{\prime}+\partial R, r^{\prime \prime}+\partial R\right]\right]=$

$$
=\left[\left[r+\partial R, r^{\prime}+\partial R\right], r^{\prime \prime}+\partial R\right]+(-1)^{p(r) p\left(r^{\prime}\right)}\left[r^{\prime}+\partial R,\left[r+\partial R, r^{\prime \prime}+\partial R\right]\right]
$$

ou seja:

$$
r_{(0)}\left(r_{(0)}^{\prime} r^{\prime \prime}\right)+\partial R=\left(r_{(0)} r^{\prime}\right)_{(0)} r^{\prime \prime}+(-1)^{p(r) p(r)} r_{(0)}^{\prime}\left(r_{(0)} r^{\prime \prime}\right)+\partial R
$$

Usando o axioma $\left(C_{4}\right)$ com $m=n=0$, temos: 


$$
\begin{aligned}
r_{(0)}\left(r_{(0)}^{\prime} r^{\prime \prime}\right) & =\sum_{j=0}^{0}\left(\begin{array}{l}
0 \\
j
\end{array}\right)\left(r_{(j)} r^{\prime}\right)_{(0+0-j)} r^{\prime \prime}+(-1)^{p(r) p\left(r^{\prime}\right)} r_{(0)}^{\prime}\left(r_{(0)} r^{\prime \prime}\right) \\
& =\left(r_{(0)} r^{\prime}\right)_{(0)} r^{\prime \prime}+(-1)^{p(r) p\left(r^{\prime}\right)} r_{(0)}^{\prime}\left(r_{(0)} r^{\prime \prime}\right)
\end{aligned}
$$

Logo $R / \partial R$ é uma superálgebra de Lie. Agora só nos resta provar $(v)$ e $(v i)$ :

$(r+\partial R)_{\circ} r^{\prime}=r_{(0)} r^{\prime}$

é, de fato, uma ação de $R / \partial R \rightarrow R$ que está bem-definida, pois:

$$
\begin{aligned}
((r+\partial a)+\partial R)_{\circ} r^{\prime} & =(r+\partial a)_{(0)} r^{\prime} \\
& =r_{(0)} r^{\prime}+(\partial a)_{(0)} r^{\prime} \\
& =r_{(0)} r^{\prime}+0
\end{aligned}
$$

Para terminar:

$$
\begin{aligned}
\partial\left((r+\partial R)_{\circ} r^{\prime}\right) & =\partial\left(r_{(0)} r^{\prime}\right) \\
& =r_{(0)}\left(\partial r^{\prime}\right) \\
& =(r+\partial R)_{\circ} f(\partial) r
\end{aligned}
$$

Assim, $\forall f(\partial) \in \mathbb{C}[\partial]$, temos $f(\partial)\left((r+\partial R)_{\circ} r^{\prime}\right)=(r+\partial R)_{\circ} f(\partial) r$, o que demonstra o teorema.

Definição 3.6. Sejam $R$ e $S$ álgebras de Lie conformes sobre $\mathbb{C}$. Uma função $\varphi: R \rightarrow S$ é chamada de homomorfismo de álgebras de Lie conformes se $\varphi$ for um homomorfismo de $\mathbb{C}[\partial]$-módulos e:

$$
\varphi\left(a_{(n)} b\right)=\varphi(a)_{(n)} \varphi(b)
$$

para todos os $a, b \in R$ e $n \geq 0$.

Também um $\mathbb{C}[\partial]$-submódulo $I$ de $R$ é chamado um ideal (à esquerda) de $R$ se $a_{(n)} b \in I$ para todo $a \in R, b \in I$ e $n \geq 0$. Como vimos na demonstração do Teorema 3.5, o axioma $\left(C_{4}\right)$ implica que todo ideal é bilateral. O centro de $R$, denotado por $Z(R)$, é a coleção dos elementos centrais de $R$. Pelo axioma $\left(C_{4}\right)$, este é um ideal de $R$. Um ideal $I$ de $R$ é dito central se $I \subset Z(R)$ e é dito comutativo se $a_{(n)} b=0$, para todo $a, b \in I$ e $n \geq 0$. A álgebra conforme $R$ é dita comutativa se for um ideal comutativo de si mesma. A coleção de todos os $\mathbb{C}[\partial]$-módulos e seus homomorfismos formam 
uma categoria. Desta forma, podemos ver que a coleção das álgebras de Lie conforme e seus homomorfismos formam uma categoria que denota-se por $\mathfrak{L} C$.

Para finalizar, observe que um método eficaz para se descrever o $n$-ésimo produto de uma superálgebra conforme $R$ é introduzir o $\lambda$-colchete. Então:

Definição 3.7. Seja $R$ um $\mathbb{C}[\partial]$-módulo munido de uma família de produtos bilineares como na definição 3.4, e seja $\lambda$ uma indeterminada. Defina o $\lambda$ colchete em $R$ por

$$
\left[a_{\lambda} b\right]=\sum_{j \geq 0} \lambda^{(j)} a_{(j)} b
$$

para todo $a, b \in R$. à:

Com esta notação, tem-se que os axiomas $\left(C_{1}\right)-\left(C_{4}\right)$ são equivalentes

Proposição 3.8. Seja $R$ um $\mathbb{C}[\partial]$-módulo munido de uma família de produtos bilineares como na definição 3.4. Então $R$ é uma superálgebra conforme sobre $\mathbb{C}$ se e somente se:

$\left(C_{1}\right)_{\lambda}\left[a_{\lambda} b\right] \in R[\lambda]$

$\left(C_{2}\right)_{\lambda}\left[\partial a_{\lambda} b\right]=-\lambda\left[a_{\lambda} b\right]$

$\left(C_{3}\right)_{\lambda}\left[a_{\lambda} b\right]=-(-1)^{p(a) p(b)}\left[b_{-\lambda-\partial} a\right]$

$\left(C_{4}\right)_{\lambda}\left[a_{\lambda}\left[b_{\mu} c\right]\right]-(-1)^{p(a) p(b)}\left[b_{\mu}\left[a_{\lambda} c\right]\right]=\left[\left[a_{\lambda} b\right]_{\lambda+\mu} c\right]$

$\left(C_{2}\right)_{\lambda}^{\prime}\left[a_{\lambda} \partial b\right]=(\partial+\lambda)\left[a_{\lambda} b\right]$

para algumas indeterminadas $\lambda, \mu$ e para $a, b, c \in R$.

Demonstração. Sabemos que $\left[a_{\lambda} b\right]=\sum_{n \geq 0} \lambda^{(n)} a_{(n)} b$. Logo se $a_{(n)} b=0$ para $n \gg 0$, então:

$$
\left[a_{\lambda} b\right]=\sum_{j=0}^{n-1} \lambda^{(j)} a_{(j)} b \in R[\lambda]
$$

e $\operatorname{assim}\left(C_{1}\right) \Rightarrow\left(C_{1}\right)_{\lambda}$. Por outro lado, se $\left[a_{\lambda} b\right] \in R[\lambda]$, então $\exists n \in \mathbb{N}$ 
tal que $a_{(m)} b=0, \forall m \geq n$. Logo $\left(C_{1}\right)_{\lambda} \Rightarrow\left(C_{1}\right)$. Agora:

$$
\left[\partial a_{\lambda} b\right]=\sum_{n=0}^{\infty} \lambda^{(n)}\left(\partial a_{(n)} b\right)
$$

e por outro lado,

$$
\begin{aligned}
-\lambda\left[a_{\lambda} b\right] & =-\lambda \sum_{n=0}^{\infty} \lambda^{(n)}\left(a_{(n)} b\right) \\
& =\sum_{n=0}^{\infty} \frac{\lambda \cdot \lambda^{n}}{(n+1) !} \cdot\left(-(n+1) \cdot a_{(n)} b\right) \\
& =\sum_{n=0}^{\infty} \lambda^{(n+1)}\left(-(n+1) a_{(n)} b\right) \\
& =\sum_{n=1}^{\infty} \lambda^{(n)}\left(-n \cdot a_{(n-1)} b\right) \\
& =\sum_{n=0}^{\infty} \lambda^{(n)}\left(-n \cdot a_{(n-1)} b\right)
\end{aligned}
$$

Logo se $\partial a_{(n)} b=-n \cdot a_{(n-1)} b$, então:

$$
\left[\partial a_{\lambda} b\right]=-\lambda\left[a_{\lambda} b\right]
$$

e se $\left[\partial a_{\lambda} b\right]=-\lambda\left[a_{\lambda} b\right]$, então

$$
\sum_{n=0}^{\infty} \lambda^{(n)}\left(\partial a_{(n)} b\right)=\sum_{n=0}^{\infty} \lambda^{(n)}\left(-n \cdot a_{(n-1)} b\right)
$$

e, desta forma, $\partial a_{(n)} b=-n \cdot a_{(n-1)} b$. Agora $\left(C_{3}\right) \Leftrightarrow\left(C_{3}\right)_{\lambda}$, pois:

$$
\left[a_{\lambda} b\right]=\sum_{n=0}^{\infty} \lambda^{(n)}\left(a_{(n)} b\right)
$$

enquanto que: 


$$
\begin{aligned}
{\left[b_{-\partial-\lambda} a\right] } & =\sum_{s \geq 0}(-1)^{s}(\partial+\lambda)^{(s)} b_{(s)} a \\
& =\sum_{s \geq 0}(-1)^{s} \cdot \frac{1}{s !}\left(\sum_{k=0}^{s}\left(\begin{array}{l}
s \\
k
\end{array}\right) \lambda^{k} \partial^{s-k}\right) b_{(s)} a \\
& =\sum_{s \geq 0}(-1)^{s}\left(\sum_{k=0}^{s} \lambda^{(k)} \partial^{(s-k)}\right) b_{(s)} a \\
& =\sum_{n, j \geq 0}(-1)^{n+j} \lambda^{(n)} \partial^{(j)} b_{(n+j)} a
\end{aligned}
$$

O resultado segue como em $\left(C_{2}\right) \Leftrightarrow\left(C_{2}\right)_{\lambda}$. Agora provemos que $\left(C_{4}\right) \Leftrightarrow\left(C_{4}\right)_{\lambda}$ :

$$
\begin{aligned}
{\left[\left[a_{\lambda} b\right]_{\lambda+\mu} c\right] } & =\sum_{i \geq 0}(\lambda+\mu)^{(i)}\left(\sum_{j \geq 0} \lambda^{(j)} a_{(j)} b\right)_{(i)} c \\
& =\sum_{i, j \geq 0} \frac{1}{i ! j !} \sum_{k=0}^{i}\left(\begin{array}{c}
i \\
k
\end{array}\right) \lambda^{i+j-k} \mu^{k}\left(a_{(j)} b\right)_{(i)} c \\
& =\sum_{m, n \geq 0} \lambda^{m} \mu^{n} \sum_{j=0}^{m+n} \frac{1}{j !(m+n-j) !}\left(\begin{array}{c}
m+n-j \\
n
\end{array}\right)\left(a_{(j)} b\right)_{(m+n-j)} c \\
& =\sum_{m, n \geq 0} \lambda^{(m)} \mu^{(n)} \sum_{j=0}^{m+n} \frac{m ! n !}{j !(m+n-j) !}\left(\begin{array}{c}
m+n-j \\
n
\end{array}\right)\left(a_{(j)} b\right)_{(m+n-j)} c \\
& =\sum_{m, n \geq 0} \lambda^{(m)} \mu^{(n)} \sum_{j=0}^{m}\left(\begin{array}{c}
m \\
j
\end{array}\right)\left(a_{(j)} b\right)_{(m+n-j)} c
\end{aligned}
$$

e também temos que:

$$
\begin{aligned}
{\left[a_{\lambda}\left[b_{\mu} c\right]\right]-(-1)^{p(a) p(b)}\left[b_{\mu}\left[a_{\lambda} c\right]\right] } & =\sum_{m \geq 0} \lambda^{(m)} a_{(m)}\left(\sum_{n \geq 0} \mu^{(n)} b_{(n)} c\right) \\
& -(-1)^{p(a) p(b)} \sum_{n \geq 0} \mu^{(n)} b_{(n)}\left(\sum_{m \geq 0} \lambda^{(m)} a_{(m)} c\right) \\
& =\sum_{m, n \geq 0} \lambda^{(m)} \mu^{(n)}\left(a_{(m)}\left(b_{(n)} c\right)\right. \\
& \left.-(-1)^{p(a) p(b)} b_{(n)}\left(a_{(m)} c\right)\right)
\end{aligned}
$$

Agora uma aplicação do $\lambda$-colchete é o seguinte Corolário: 
Corolário 3.9. Todo elemento de torção a de uma superálgebra conforme finita $R$ é central (onde um elemento $\underline{a}$ é dito de torção se $\exists p(x) \in \mathbb{C}[\partial]$ tal que $p(\partial) a=0)$. Em particular, se $R$ é finita, então como um $\mathbb{C}[\partial]$-módulo, $R$ é uma soma direta de uma subálgebra central de dimensão finita (sobre $\mathbb{C}$ ) e um $\mathbb{C}[\partial]$-módulo livre de posto finito.

Demonstração. Por definição, temos que $p(\partial) a=0$ para algum polinômio $p(x)$, então $\left[p(\partial) a_{\lambda} b\right]=0$ para todo $b \in R$ e por $\left(C_{1}\right)_{\lambda}$, temos que $p(-\lambda)\left[a_{\lambda} b\right]=0$. Como $p(-\lambda)$ é um polinômio não-nulo em $\lambda$ e $\left[a_{\lambda} b\right]$ não possui em sua expansão termos negativos, então $\left[a_{\lambda} b\right]=0$ para todo $b \in R$, $\operatorname{logo}^{a}$ é um elemento central. Agora analisando $R$ como um $\mathbb{C}[\partial]$-módulo, temos que $R$ é finita se, e somente se, $R$ é finitamente gerada como um $\mathbb{C}[\partial]$-módulo, isto é, se existem $r_{1}, r_{2}, \ldots, r_{n} \in R$ tais que:

$$
R=\mathbb{C}[\partial] r_{1}+\ldots+\mathbb{C}[\partial] r_{n}
$$

Reordenando os elementos, se necessário, podemos assumir que $r_{1}, \ldots, r_{m}$ são elementos de torção e $r_{m+1}, \ldots, r_{n}$ não são. Se $\mathbb{C}[\partial] r_{m+1}+\ldots+\mathbb{C}[\partial] r_{n}$ não possui elementos de torção, então acabou. Caso $\exists r_{m+1}^{\prime}$ elemento de torção, então existe um $j \in m+1, \ldots, n$ tal que:

$\mathbb{C}[\partial] r_{m+1}+\ldots+\mathbb{C}[\partial] r_{n}=\mathbb{C}[\partial] r_{m+1}+\ldots+{\widehat{\mathbb{C}}[\partial] r_{j}}_{+}+\ldots+\mathbb{C}[\partial] r_{n}+\mathbb{C}[\partial] r_{m+1}^{\prime}$

Sem perda de generalidade, podemos supor que $j=m+1$ e trocar $r_{m+1}$ por $r_{m+1}^{\prime}$. Se $\nexists r_{m+2}^{\prime} \in \mathbb{C}[\partial] r_{m+2}+\ldots+\mathbb{C}[\partial] r_{n}$, então acabou. Caso contrário, repita o argumento. Este processo terminará em algum $k$, com $m \leq k \leq n$. Assim, $R=R_{1}+R_{2}$ onde:

$$
\begin{gathered}
R_{1}=\mathbb{C}[\partial] r_{1}+\ldots+\mathbb{C}[\partial] r_{k} \\
R_{2}=\mathbb{C}[\partial] R_{k+1}+\ldots+\mathbb{C}[\partial] r_{n}
\end{gathered}
$$

Agora temos que $R_{1} \cap R_{2}=\{0\}$, pois todos os elementos não nulos de $R_{1}$ são de torção enquanto que os de $R_{2}$ não são de torção. $\operatorname{Logo} R=R_{1} \oplus R_{2}$, onde $R_{1}$ é uma subalgébra central de dimensão finita sobre $\mathbb{C}$ e $R_{2}$ é um $\mathbb{C}[\partial]$-módulo livre de posto finito.

Teorema 3.10. Se $\mathfrak{g}$ é gerada (via n-ésimos produtos) pelos coeficientes de uma coleção $F$ de distribuições formais mutuamente locais, então $\bar{F}$ é uma superálgebra conforme.

Demonstração. $\bar{F}$ é, por definição, o $\mathbb{C}[\partial]$-módulo minimal de $f d(\mathfrak{g})$ fechado sobre todos os $n$-ésimos produtos (e contendo $F$ ), com $n \in \mathbb{Z}_{+}$. Sendo assim, para provar que $\bar{F}$ é uma superálgebra conforme, basta mostrarmos 
que com o $\mathbb{C}$-produto bilinear $a_{(n)} b$, para todo $n \in \mathbb{Z}_{+}$, os axiomas $\left(C_{1}\right),\left(C_{2}\right),\left(C_{3}\right)$ e $\left(C_{4}\right)$ valem, com $a, b, c \in \bar{F}$ e $m, n \in \mathbb{Z}_{+}$. De fato, vale $\left(C_{1}\right)$ pois:

$$
a(w)_{(n)} b(w)=\operatorname{Res}_{z}[a(z), b(w)] \cdot(z-w)^{n}
$$

Como $a(z)$ e $b(w)$ são mutuamente locais, então $\exists m>0$ tal que:

$$
\begin{aligned}
& {[a(z), b(w)] \cdot(z-w)^{m}=0, \operatorname{logo:}} \\
& \begin{aligned}
a(w)_{(m)} b(w) & =\operatorname{Res}_{z} 0 \\
& =0
\end{aligned}
\end{aligned}
$$

Assim, seja $a(w)=a$, isto é, $a(w)=a+\sum_{n \in \mathbb{Z}^{*}} 0 \cdot w^{n}$, e seja $b(w)=b$. Temos que $a_{(m)} b=0$. Agora, provemos $\left(C_{2}\right)$. Sabemos que $\partial a(w)_{(n)} b(w)=$ $-n \cdot a(w)_{(n-1)} b(w)$ pelo Teorema 2.15(i). Tomando $a(w)=a$ e $b(w)=b$, segue $\left(C_{2}\right)$. Agora $\left(C_{3}\right)$ e $\left(C_{4}\right)$ seguem analogamente ao Teorema 2.15 , ítens (iii) e $(i v)$.

Agora será construída uma superálgebra de Lie de distribuições formais, denotada por LieR, associada à superálgebra conforme $R$ do seguinte modo:

Definição 3.11. Seja LieR o quociente do espaço vetorial com elementos de base $a_{n}(a \in R, n \in \mathbb{Z})$ pelo subespaço gerado sobre $\mathbb{C}$ pelos elementos:

$(\lambda a)_{n}-\lambda a_{n} ;(a+b)_{n}-a_{n}-b_{n} ;(\partial a)_{n}+n \cdot a_{n-1}$

onde $a, b \in R, \lambda \in \mathbb{C}, n \in \mathbb{Z}$.

Aqui é válida uma fórmula similar a (3.1), dada pelo colchete em LieR:

$$
\left[a_{m}, b_{n}\right]=\sum_{j \in \mathbb{Z}_{+}}\left(\begin{array}{c}
m \\
j
\end{array}\right)\left(a_{(j)} b\right)_{m+n-j}
$$

Existe uma maneira alternativa de se definir LieR. Devido a sua importância na hora de fazer os cálculos, será feita tal construção, mas antes, faz-se necessária algumas definições: 
Definição 3.12. Defina como afinizador de uma superálgebra conforme $R$ a superálgebra conforme $\tilde{R}=R\left[t, t^{-1}\right]$, com $p(t)=0$ e $\tilde{\partial}=\partial \otimes 1+1 \otimes \partial_{t}$, e o $n$-ésimo produto definido por:

$$
(a \otimes f)_{(n)}(b \otimes g)=\sum_{j \in \mathbb{Z}_{+}}\left(a_{(n+j)} b\right) \otimes\left(\left(\partial_{t}^{(j)} f\right) g\right)
$$

onde $a, b \in R ; f, g \in \mathbb{C}\left[t, t^{-1}\right], n \in \mathbb{Z}_{+}$.

Seja $a_{n}=a \otimes t^{n}$, então a fórmula (3.5) fica:

$$
\left(a_{m}\right)_{(k)}\left(b_{n}\right)=\sum_{j \in \mathbb{Z}_{+}}\left(\begin{array}{c}
m \\
j
\end{array}\right)\left(a_{(k+j)} b\right)_{m+n-j}
$$

De fato,

$$
\begin{aligned}
\left(a_{m}\right)_{(k)}\left(b_{n}\right) & =\left(a \otimes t^{m}\right)_{(k)}\left(b \otimes t^{n}\right) \\
& =\sum_{j \in \mathbb{Z}_{+}}\left(a_{(k+j)} b\right) \otimes\left(\left(\partial_{t}^{(j)} t^{m}\right) t^{n}\right) \\
& =\sum_{j \in \mathbb{Z}_{+}}\left(a_{(k+j)} b\right) \otimes\left(\left(\left(\begin{array}{c}
m \\
j
\end{array}\right) t^{m-j}\right) t^{n}\right) \\
& =\sum_{j \in \mathbb{Z}_{+}}\left(\begin{array}{c}
m \\
j
\end{array}\right)\left(a_{(k+j)} b\right)_{m+n-j}
\end{aligned}
$$

Seja LieR $=\tilde{R} / \tilde{\partial} \tilde{R}$ com o colchete induzido pelo 0-ésimo produto em $\tilde{R}$. Assim, obtêm-se uma superálgebra de Lie que é a mesma que a introduzida anteriormente, bastando considerar o isomorfismo $\varphi$ que leva $a_{n}$ em $\overline{a \otimes t^{n}}$. Agora resta checar que, realmente, $\tilde{R}$ é uma superálgebra conforme para que as observações acima estejam realmente corretas, mas antes de mais nada, temos que o correspondente $\lambda$-colchete é dado por:

$$
\left[a \otimes f_{\lambda} b \otimes g\right]=\left.\left[a_{\lambda+\partial_{t}} b\right] \otimes f(t) g\left(t^{\prime}\right)\right|_{t^{\prime}=t}
$$

De fato, 


$$
\begin{aligned}
{\left.\left[a_{\lambda+\partial_{t}} b\right] \otimes f(t) g\left(t^{\prime}\right)\right|_{t^{\prime}=t} } & =\left.\sum_{n \geq 0} \frac{1}{n !}\left(\lambda+\partial_{t}\right)^{n} a_{(n)} b \otimes f(t) g\left(t^{\prime}\right)\right|_{t^{\prime}=t} \\
& =\left.\sum_{n \geq 0} \sum_{j \geq 0} \frac{1}{n !} \cdot \frac{n !}{(n-j) ! j !} \lambda^{n-j} \partial_{t}^{j} a_{(n)} b \otimes f(t) g\left(t^{\prime}\right)\right|_{t^{\prime}=t} \\
& =\left.\sum_{j \geq 0} \sum_{n \geq 0} \lambda^{(n-j)} \partial_{t}^{(j)} a_{(n)} b \otimes f(t) g\left(t^{\prime}\right)\right|_{t^{\prime}=t} \\
& =\left.\sum_{j \geq 0} \sum_{n \geq j} \lambda^{(n-j)} \partial_{t}^{(j)} a_{(n)} b \otimes f(t) g\left(t^{\prime}\right)\right|_{t^{\prime}=t} \\
& =\left.\sum_{j \geq 0} \sum_{n \geq 0} \lambda^{(n)} \partial_{t}^{(j)} a_{(n+j)} b \otimes f(t) g\left(t^{\prime}\right)\right|_{t^{\prime}=t} \\
& =\left.\sum_{n \geq 0} \lambda^{(n)} \sum_{j \geq 0} a_{(n+j)} b \otimes\left(\partial_{t}^{(j)} f(t) g\left(t^{\prime}\right)\right)\right|_{t^{\prime}=t} \\
& =\sum_{n \geq 0} \lambda^{(n)}\left(a \otimes f_{(n)} b \otimes g\right) \\
& =\left[a \otimes f_{\lambda} b \otimes g\right]
\end{aligned}
$$

Teorema 3.13. $\tilde{R}$, como na Definição 3.12, é uma superálgebra conforme.

Demonstração. Basta provar $\left(C_{1}\right)_{\lambda},\left(C_{2}\right)_{\lambda},\left(C_{3}\right)_{\lambda}$ e $\left(C_{4}\right)_{\lambda}$. Aqui, provaremos $\left(C_{2}\right)_{\lambda}$ e $\left(C_{3}\right)_{\lambda}$, deixando os outros dois a cargo do leitor:

$$
\begin{aligned}
{\left[\tilde{\partial}(a \otimes f)_{\lambda} b_{\otimes} g\right] } & =\left[\partial(a \otimes f)_{\lambda} b_{\otimes} g\right]+\left[a \otimes \partial_{t} f_{\lambda} b_{\otimes} g\right] \\
& =\left[\partial a_{\lambda+\partial_{t}} b\right] \otimes f(t) g\left(t^{\prime}\right)+\left.\left[a_{\lambda+\partial_{t}} b\right] \otimes \partial_{t} f(t) g\left(t^{\prime}\right)\right|_{t^{\prime}=t} \\
& =-\left(\lambda+\partial_{t}\right)\left[a_{\lambda+\partial_{t}} b\right] \otimes f(t) g\left(t^{\prime}\right)+\partial_{t}\left(\left.\left[a_{\lambda+\partial_{t}} b\right] \otimes f(t) g\left(t^{\prime}\right)\right|_{t^{\prime}=t}\right. \\
& =-\left.\lambda\left[a_{\lambda+\partial_{t}} b\right] \otimes f(t) g\left(t^{\prime}\right)\right|_{t^{\prime}=t} \\
& =-\lambda\left[a \otimes f_{\lambda} b \otimes g\right]
\end{aligned}
$$

Logo vale $\left(C_{2}\right)_{\lambda}$. Agora provemos $\left(C_{3}\right)_{\lambda}$ :

$$
\begin{aligned}
{\left[a \otimes f_{\lambda} b \otimes g\right] } & =\left.\left[a_{\lambda+\partial_{t}} b\right] \otimes f(t) g\left(t^{\prime}\right)\right|_{t^{\prime}=t} \\
& =-\left.(-1)^{p(a) p(b)}\left[b_{-\lambda-\partial_{t}-\partial} a\right] \otimes f(t) g\left(t^{\prime}\right)\right|_{t^{\prime}=t} \\
& =-\left.(-1)^{p(a) p(b)}\left[b_{-\lambda-\partial-\partial_{t}-\partial_{t^{\prime}}+\partial_{t^{\prime}}} a\right] \otimes g\left(t^{\prime}\right) f(t)\right|_{t=t^{\prime}} \\
& =-(-1)^{p(a) p(b)}\left[b \otimes g_{-\lambda-\partial-\partial_{t}-\partial_{t^{\prime}}} a \otimes f\right] \\
& =-(-1)^{p(a) p(b)}\left[b \otimes g_{-\lambda-\partial-\partial_{t^{\prime}}} a \otimes f\right] \\
& =-(-1)^{p(a) p(b)}\left[b \otimes g_{-\lambda-\tilde{\partial}} a \otimes f\right]
\end{aligned}
$$

Temos também que $-1 \otimes \partial_{t}$ é uma derivação do 0-ésimo produto da superálgebra conforme $\tilde{R}$. Como este operador comuta com $\tilde{\partial}$, este induz 
uma derivação $T$ da superálgebra de Lie LieR dada pela fórmula:

$$
T\left(a_{n}\right)=-n \cdot a_{n-1}
$$

Isto de fato ocorre, pois por (3.6), temos que:

$$
\begin{aligned}
\left(-1 \otimes \partial_{t}\right)\left(\sum_{j \in \mathbb{Z}_{+}}\left(a_{(j)} b\right) \otimes\left(\partial_{t}^{(j)} f\right) g\right) & =-\sum_{j \in \mathbb{Z}_{+}}\left(a_{(j)} b\right) \otimes \partial_{t}\left(\left(\partial_{t}^{(j)} f\right) g\right) \\
& =-\sum_{j \in \mathbb{Z}_{+}}\left(a_{(j)} b\right) \otimes\left(\partial_{t}^{(j)} \partial_{t} f\right) g \\
& -\sum_{j \in \mathbb{Z}_{+}}\left(a_{(j)} b\right) \otimes\left(\partial_{t}^{(j)} f\right) \partial_{t} g \\
& =\left(-a \otimes f^{\prime}\right)_{(0)}(b \otimes g)-(a \otimes f)_{(0)}(b \otimes g)
\end{aligned}
$$

Desta forma, seja $\partial: \tilde{R} \rightarrow \tilde{R}$. Sabemos que $\forall a_{n} \in \tilde{R}, \overline{\tilde{\partial} a_{n}}=\overline{0} \in$ LieR. Assim:

$$
\begin{aligned}
\overline{\left(\partial \otimes 1+1 \otimes \partial_{t}\right) a_{n}}=\overline{0} & \Rightarrow \overline{(\partial \otimes 1) a \otimes t^{n}}=\overline{\left(-1 \otimes \partial_{t}\right) a \otimes t^{n}} \\
& \Rightarrow \overline{\partial a \otimes t^{n}}=\overline{-n \cdot a \otimes t^{n-1}}
\end{aligned}
$$

Logo, $\partial a_{n}=-n \cdot a_{n-1}$ em LieR e assim, como $\left(-1 \otimes \partial_{t}\right)$ comuta com $\tilde{\partial}$, temos que se $a_{n}+\tilde{\partial} b \in \operatorname{Lie} R$, então:

$$
\begin{aligned}
T\left(\bar{a}_{n}\right) & =\partial\left(a_{n}+\tilde{\partial} b\right) \\
& =\partial\left(a_{n}\right)+\tilde{\partial}(\partial b) \\
& =-n \cdot a_{n-1}+\tilde{\partial} c \\
& =\frac{-n \cdot a_{n-1}}{\mathrm{em} \mathrm{LieR}}
\end{aligned}
$$

Assim, $\partial$ induz a derivação $T$ (onde $\partial: \tilde{R} \rightarrow \tilde{R}$ e também, $T:$ Lie $R \rightarrow$ LieR).

Teorema 3.14. Seja $R$ uma superálgebra conforme e suponha que como um $\mathbb{C}[\partial]$-módulo, $R \simeq(\mathbb{C}[\partial] \otimes V) \oplus C$, onde $V$ é um espaço vetorial (sobre $\mathbb{C}$ ) $e$ $C$ consiste de elementos de torção. Então, como espaço vetorial, temos que Lie $R \simeq V\left[t, t^{-1}\right] \oplus C^{\prime}$, onde $C^{\prime}$ é uma subálgebra central de LieR.

Demonstração. Basta observar que isto vale nos dois casos abaixo: 
(1) $\operatorname{dim}_{\mathbb{C}} V=1$ e $C=0$

(2) $V=0$ e $\operatorname{dim}_{\mathbb{C}} C=1$

Como $\tilde{R}=R \otimes \mathbb{C}\left[t, t^{-1}\right]$, então temos que:

$$
\begin{aligned}
\tilde{R} & =(\mathbb{C}[\partial] \otimes V) \otimes \mathbb{C}\left[t, t^{-1}\right] \oplus C \otimes \mathbb{C}\left[t, t^{-1}\right] \\
& =\mathbb{C}[\partial] \otimes V\left[t, t^{-1}\right] \oplus C \otimes \mathbb{C}\left[t, t^{-1}\right] \\
& =\tilde{\partial} \tilde{R}+\left(V\left[t, t^{-1}\right] \oplus C\right)
\end{aligned}
$$

Agora vale a igualdade, pois qualquer $\partial a$ é igual a $\tilde{\partial} \tilde{a}+a^{\prime}$, para algum $a^{\prime}$. De fato, $\partial(v \otimes 1)=\tilde{\partial}(v \otimes 1) \in \tilde{\partial} \tilde{R}$. Agora suponhamos válido para $\partial\left(v \otimes t^{n-1}\right)$ e provemos para $\partial\left(v \otimes t^{n}\right)$. Temos:

$$
\begin{aligned}
\partial\left(v \otimes t^{n}\right) & =\tilde{\partial}\left(v \otimes t^{n}\right)-\left(v \otimes \partial_{t} t^{n}\right) \\
& =\tilde{\partial}\left(v \otimes t^{n}\right)-n \cdot\left(v \otimes t^{n-1}\right)
\end{aligned}
$$

Assim, para $a=v_{n}$, temos $a^{\prime}=-n \cdot v_{n-1}$. Analogamente, $C \otimes \mathbb{C}\left[t, t^{-1}\right] \subset$ $\tilde{\partial} \tilde{R} \oplus C$. Assim, $V\left[t, t^{-1}\right] \oplus C$ é complementado em $\tilde{R}$ por $\tilde{\partial} \tilde{R}$ e assim, como espaço vetorial (sobre $\mathbb{C}$ ), temos que:

$$
\text { Lie } R \simeq V\left[t, t^{-1}\right] \oplus C
$$

Agora o resultado segue, pois $V=\oplus \mathbb{C} v_{i}$ e $C=\oplus \mathbb{C} c_{j}$. Assim:

$$
\text { Lie } R \simeq\left(\oplus \mathbb{C} v_{i}\left[t, t^{-1}\right]\right) \oplus\left(\oplus \mathbb{C} c_{j}\right)
$$

Lema 3.15. Se $a \in R$ e os elementos $a_{-1} \in$ LieR são nulos, então $a=0$. Em particular, $\varphi$ é um isomorfismo de superálgebras conformes.

Demonstração. Definimos a aplicação linear $\tilde{\psi}: \tilde{R} \rightarrow R$ de espaços vetoriais sobre $\mathbb{C}$ por:

$\tilde{\psi}\left(a t^{-j-1}\right)=\partial^{(j)} a$, onde $j \in \mathbb{Z}_{+}$.

Então $\tilde{\psi}(\tilde{\partial} \tilde{R})=0$, pois:

$$
\begin{aligned}
\tilde{\psi}\left(\left(\partial \otimes 1+1 \otimes \partial_{t}\right) a \otimes t^{n}\right) & =\tilde{\psi}\left(\partial a \otimes t^{n-1}\right) \\
& =\tilde{\psi}\left(\partial a \otimes t^{n}\right)+n \cdot \tilde{\psi}\left(a \otimes t^{n-1}\right) \\
& =\star
\end{aligned}
$$


temos duas possibilidades:

(1) Se $n \in \mathbb{Z}_{+}$, então:

$$
\begin{aligned}
\star & =0+n \cdot 0 \\
& =0
\end{aligned}
$$

(2) Se $n \in \mathbb{Z}_{-}^{*}$, então:

$$
\begin{aligned}
\star & =\partial^{(-n-1)} \partial a+n \cdot \partial^{(n)} a \\
& =\partial^{(n-1)} \partial a-\frac{-n \cdot \partial^{-n-1} \partial a}{-n \cdot(-n-1) !} \\
& =\partial^{(-n-1)} \partial a-\partial^{(-n-1)} \partial a \\
& =0
\end{aligned}
$$

Assim, provamos que $\tilde{\psi}(\tilde{\partial} \tilde{R})=0$. Agora como LieR $=\tilde{R} / \tilde{\partial} \tilde{R}$, defina $\psi:$ Lie $R \rightarrow R$ tal que $\psi(\bar{a})=\tilde{\psi}(a)$. Esta operação está bem-definida, pois:

$$
\begin{aligned}
\psi(\overline{a+\hat{\partial} b}) & =\tilde{\psi}(a+\hat{\partial} b) \\
& =\tilde{\psi}(a)+\tilde{\psi}(\hat{\partial} b) \\
& =\tilde{\psi}(a)+0 \\
& =\tilde{\psi}(a)
\end{aligned}
$$

para todo $\hat{\partial} b \in \tilde{\partial} \tilde{R}$. Assim, $\tilde{\psi}$ induz uma aplicação $\psi$ de LieR em $R$. Agora $\psi\left(a_{-1}\right)=a$, pois:

$$
\begin{aligned}
\tilde{\psi}\left(a_{-1}\right) & =\tilde{\psi}\left(a \otimes t^{-1}\right) \\
& =\partial^{(0)} a \\
& =a \in \tilde{R}
\end{aligned}
$$

Logo $\psi\left(\hat{a}_{-1}\right)=\tilde{\psi}\left(a_{-1}\right)=a$, com $\hat{a}_{-1} \in$ LieR. Assim, se $a_{-1} \in \operatorname{LieR}$ é zero, então $a=0$, pois toda aplicação linear leva zero em zero. Isto implica que $\varphi$ é isomorfismo de superálgebras conformes, pois $\varphi$ já é um homomorfismo sobrejetor e $\varphi$ é injetor pois $\varphi(a)=0 \Rightarrow a=0$. De fato:

$$
\begin{aligned}
\varphi(a) & =a(z) \\
& =\sum_{n \in \mathbb{Z}} a_{n} z^{-n-1}
\end{aligned}
$$


Assim, $\varphi(a)=0 \Rightarrow a_{n}=0, \forall n \in \mathbb{Z}$ implica que $a_{-1}=0$ que implica que $a=0$.

\subsection{A equivalência entre a categoria das superálgebras conformes e a categoria das classes de equivalência das superálgebras de Lie de distribuições formais}

Definição 3.16. Dada uma superálgebra de Lie de distribuições formais $(\mathfrak{g}, F)$ sobre $\mathbb{C}$, considere a ação do símbolo $\partial$ em $\bar{F}$ dado por $(\partial a)(z)=$ $\partial a(z)=\partial_{z} a(z)$, para todo $a \in \bar{F}$.

Chame o $K[\partial]$-módulo $K[\partial] \bar{F}$ de conformalizador do par $(\mathfrak{g}, F)$. Daí resulta que o conformalizador de um par é uma superálgebra de Lie conforme, com família de produtos dados pelos produtos indexados de distribuições formais. Um dos objetivos desta seção é expressar o processo de conformalização na linguagem da teoria de categorias, como um funtor. Então dada uma superálgebra de Lie de distribuições formais $(\mathfrak{g}, F)$, seja $\operatorname{Con}(\mathfrak{g}, F)$ o seu conformalizador. O objeto $(\mathfrak{g}, F)$ reside na categoria $\mathfrak{L} F D$ (das superálgebras de Lie de distribuições formais), enquanto que $\operatorname{Con}(\mathfrak{g}, F)$ reside na categoria $\mathfrak{L} C$ (das superálgebras conformes). Suponha agora que $\phi:\left(\mathfrak{g}_{1}, F_{1}\right) \rightarrow\left(\mathfrak{g}_{2}, F_{2}\right)$ seja um homomorfismo de superálgebras de Lie de distribuições formais. Então:

Definição 3.17. Defina o conformalizador de $\phi$, e o denote por $C o n(\phi)$, a ação coeficiente a coeficiente de $\phi$ nos membros de Con $\left(\mathfrak{g}_{1}, F_{1}\right)=K[\partial] \bar{F}_{1}$. Em outras palavras, $\operatorname{Con}(\phi): \operatorname{Con}\left(\mathfrak{g}_{1}, F_{1}\right) \rightarrow \operatorname{Con}\left(\mathfrak{g}_{2}, F_{2}\right)$ é tal que:

$$
\operatorname{Con}(\phi): a(z)=\sum_{n \in \mathbb{Z}} a_{n} z^{n} \mapsto \sum_{n \in \mathbb{Z}} \phi\left(a_{n}\right) z^{n}
$$

para todos os $a(z) \in K[\partial] \bar{F}_{1}$

O conformalizador $\operatorname{Con}(\phi)$ é claramente $\mathbb{C}$-linear. Além disso:

$$
\begin{aligned}
\operatorname{Con}(\phi)(\partial a(z)) & =\operatorname{Con}(\phi)\left(\sum_{n \in \mathbb{Z}} n \cdot a_{n} z^{n-1}\right) \\
& =\sum_{n \in \mathbb{Z}} \phi\left(n \cdot a_{n}\right) z^{n-1} \\
& =\partial \sum_{n \in \mathbb{Z}} \phi\left(a_{n}\right) z^{n} \\
& =\partial \operatorname{Con}(\phi)(a(z))
\end{aligned}
$$


e assim, $\operatorname{Con}(\phi)$ é $\mathbb{C}[\partial]$-linear. A equação:

$$
a_{(n)} b=\sum_{k=0}^{n}(-1)^{k}\left(\begin{array}{l}
n \\
k
\end{array}\right) \sum_{i, j \in \mathbb{Z}}\left[a_{(i-n)+k}, b_{j-k}\right] \cdot z^{i} w^{j}
$$

dá uma expressão linear do $j$-ésimo produto de dois pares locais de distribuições formais, com o qual segue que $\operatorname{Con}(\phi)$ preserva o produto indexado, e é este então um homomorfismo de superálgebras de Lie conformes.

É imediato da definição de conformalizador de um homomorfismo que este preserva composição e manda homomorfismo identidade em homomorfismo identidade. Segue que Con é, de fato, um funtor da categoria $\mathfrak{L} F D$ para a categoria $\mathfrak{L} C$.

Definição 3.18. Seja $(\mathfrak{g}, F)$ uma superálgebra de Lie de distribuições formais e seja I um ideal de $\mathfrak{g}$. O ideal I é chamado de regular se ele contém todos os coeficientes de algum elemento de $K[\partial] \bar{F}$. Caso contrário, é dito irregular.

Seja $\pi: \mathfrak{g} \rightarrow \mathfrak{g} / I$ a projeção canônica. Denote por $F / I$ a imagem de $F$ por $\pi$. Então os coeficientes dos termos de $F / I$ geram $\mathfrak{g} / I$ e estes são pares locais. Então $(\mathfrak{g} / I, F / I)$ é uma superálgebra de Lie de distribuições formais, a qual será chamada de o quociente de $(\mathfrak{g}, F)$ por $I$. Desta forma, é evidente pela definição da família $F / I$ que:

$$
\operatorname{Con}(\pi): \operatorname{Con}(\mathfrak{g}, F) \rightarrow \operatorname{Con}(\mathfrak{g} / I, F / I)
$$

é uma sobrejeção, e é imediato de nossa definição que a injetividade vale se, e somente se, o ideal $I$ é irregular. Assim, Con não é um funtor fiel: se $I$ é irregular e não-nulo, então $(\mathfrak{g}, F)$ e $(\mathfrak{g} / I, F / I)$ são conformemente isomorfos, mas não são isomorfos como superálgebras de Lie de distribuições formais.

A definição acima de ideal irregular difere levemente da feita em [1], onde este é definido como um ideal que não contém todos os coeficientes de um membro da família $\bar{F}$ (ou melhor, de $\mathbb{C}[\partial] \bar{F}$ ). Esta definição foi escolhida para que a afirmação feita após (3.10) valesse para todo ideal, o que não ocorre na definição de [1], como pode-se ver no exemplo abaixo:

Exemplo 3.19. Seja $\mathfrak{g}$ um espaço vetorial livre sobre $\mathbb{C}$ com os símbolos $c, d$ munidos do colchete de Lie trivial e seja $I=\mathbb{C} d$. Defina $a(z)=c+d z e$ 
$F=a(z)$, então o par $(\mathfrak{g}, F)$ é uma álgebra de Lie de distribuições formais. Vale que $[a(z), a(w)]=0$, logo todos os produtos indexados são nulos. Então $\bar{F}=\mathbb{C} F$ e I é irregular de acordo com a definição dada em [1].

Seja $\pi:(\mathfrak{g}, F) \rightarrow(\mathfrak{g} / I, F / I)$ a projeção canônica. Note que $\partial a(z)=d$ é um membro não nulo de $\mathbb{C}[\partial] \bar{F}$, e então $\operatorname{Con}(\pi): \partial a(z) \mapsto d=0(\bmod I)$, $e$ assim, $\operatorname{Con}(\pi)$ não é injetivo.

Voltando à teoria, o funtor conformalizador $C$ on da categoria $\mathfrak{L} F D$ para a categoria $\mathfrak{L} C$ não é fiel, como dito, contudo a relação entre as duas categorias é bastante estreita. Isto porque $C o n$ é essencialmente sobrejetivo, isto é, um objeto de $\mathfrak{L} C$ é isomorfo via conformalizador a um objeto de $\mathfrak{L} F D$. Além disso, considerando os isomorfismos conformes como equivalência, então existirá uma única representação "maximal" (chamamos (Lie $R, F(R))$ de superálgebra de Lie de distribuição formal maximal de $R$ ) em cada classe de equivalência (para maiores detalhes sobre maximalidade, veja [1] e [4]).

A chave para demonstrar a "equivalência" entre as duas categorias será a construção de um funtor de $\mathfrak{L} C$ para $\mathfrak{L} F D$, que tomará uma superálgebra de Lie conforme e associará sua correspondente superálgebra de Lie de distribuições formais maximal. Este funtor, o qual (por abuso de notação) também será denotado por Lie, será o inverso de um funtor (que também será denotado, por abuso de notação, por) Con, e irá requerer certo trabalho para se construir. Agora vale o seguinte:

Teorema 3.20. Seja $R$ uma superálgebra de Lie conforme, seja $(L, F)=$ LieR e seja $\pi: \tilde{R}_{0} \rightarrow L$ a projeção canônica. Então $R \simeq$ ConLieR via $a \mapsto \pi(\tilde{a}(z))$.

Demonstração. Defina $\varphi: R \rightarrow$ ConLieR por $\varphi: a \mapsto \pi(\tilde{a}(z))$ para todo $a \in R$. Então é imediato que $\varphi$ é $\mathbb{C}$-linear e sobrejetivo. Observe que $\pi(\tilde{a}(z))=\sum_{n \in \mathbb{Z}} \pi\left(a t^{n}\right) z^{-n-1}, \operatorname{logo} \pi(\tilde{a}(z))=0 \Leftrightarrow \pi\left(a t^{n}\right)=0$, para todo $n \in \mathbb{Z}$. Em particular, $\pi(\tilde{a}(z))=0 \Rightarrow \pi\left(a t^{-1}\right)=0$, o que ocorre apenas se $a=0$ pelo Lema 3.15. Assim, $\varphi$ é injetivo.

Agora nos resta mostrar que $\varphi$ é $\mathbb{C}[\partial]$-linear e preserva o produto indexado. $\tilde{R}_{0}$ é claramente um $\mathbb{C}\left[\partial, \partial_{t}\right]$-módulo. Observe que a ação de $\partial \mathrm{em}$ todo gerador $a t^{m}$ de $\tilde{R}_{0}$ depende apenas do coeficiente, enquanto que a ação de $\partial_{t}$ depende apenas do expoente. Assim, $\partial$ e $\partial_{t}$ comutam. Usando este fato,uma rápida checagem nos mostra que $\tilde{\partial} \tilde{R}_{0}$ é um $\mathbb{C}[\partial]$-módulo. Então a projeção $\pi$ é um homomorfismo de $\mathbb{C}\left[\partial, \partial_{t}\right]$-módulos. Então: 


$$
\begin{aligned}
\pi\left(\partial a t^{n}\right) & =\partial \pi\left(a t^{n}\right) \\
& =-\partial_{t} \pi\left(a t^{n}\right) \\
& =-\pi\left(\partial_{t} a t^{n}\right) \\
& =-n \cdot \pi\left(a t^{n-1}\right)
\end{aligned}
$$

para todo $a t^{n} \in \tilde{R}_{0}$, e assim:

$$
\begin{aligned}
\varphi(\partial a) & =\pi(\tilde{\partial a}) \\
& =\sum_{n \in \mathbb{Z}} \pi\left(\partial a t^{n}\right) z^{-n-1} \\
& =\sum_{n \in \mathbb{Z}}-n \cdot \pi\left(a t^{n-1}\right) z^{n-1} \\
& =\sum_{n \in \mathbb{Z}}(-n-1) \cdot \pi\left(a t^{n}\right) z^{-n-2} \\
& =\partial_{z} \pi(\tilde{a}(z)) \\
& =\partial \varphi(a)
\end{aligned}
$$

para todo $a \in R$, e assim, $\varphi$ é $\mathbb{C}[\partial]$-linear. Agora $\varphi$ preserva claramente o produto indexado.

Teorema 3.21. Seja $(\mathfrak{g}, F)$ uma superálgebra de Lie de distribuições formais sobre $\mathbb{C}$. Então $(\mathfrak{g}, F)$ é isomorfo a um quociente de LieCon $(\mathfrak{g}, F)$ por um ideal irregular.

Demonstração. Seja $(L, G)=\operatorname{LieCon}(\mathfrak{g}, F)$ e defina $\varphi:(L, G) \rightarrow(\mathfrak{g}, F)$ por $\varphi: a t^{m} \mapsto a_{(m)}$. Então $\varphi$ está bem-definida, é $\mathbb{C}$-linear e sobrejetora. Além disso, $\varphi$ é um homomorfismo de superálgebras de Lie. De fato,

$$
\begin{aligned}
{\left[\varphi\left(a t^{m}\right), \varphi\left(b t^{n}\right)\right] } & =\left[a_{(m)}, b_{(n)}\right] \\
& \stackrel{(\star)}{=} \sum_{j \geq 0}\left(\begin{array}{c}
m \\
j
\end{array}\right) a_{(j)} b_{(m+n-j)} \\
& =\sum_{j \geq 0}\left(\begin{array}{c}
m \\
j
\end{array}\right) \varphi\left(a_{(j)} b t^{m+n-j}\right) \\
& \stackrel{(\star \star)}{=} \varphi\left(\sum_{j \geq 0}\left(\begin{array}{c}
m \\
j
\end{array}\right) a_{(j)} b t^{m+n-j}\right) \\
& =\varphi\left(\left[a t^{m}, b t^{n}\right]\right)
\end{aligned}
$$

onde vale $(\star)$ pelo teorema $2.8(e)$ e também $(\star \star)$, já que a soma é finita. 
Então $\varphi$ induz um isomorfismo $L / K \operatorname{Ker} \varphi \rightarrow \mathfrak{g}$. Seja $I=\operatorname{Ker} \varphi$ e $\psi:(\mathfrak{g}, F) \rightarrow$ $(L / I, G / I)$ o inverso do isomorfismo induzido e seja $\pi:(L, G) \rightarrow(L / I, G / I)$ a projeção canônica. Seja $R=\operatorname{Con}(\mathfrak{g}, F)$. Assim, pode-se mostrar que $\operatorname{Con}(\pi):$ ConLieR $=\operatorname{Con}(L, G) \rightarrow \operatorname{Con}(L / I, G / I)$ é injetivo como já dito em (3.10). Temos:

$$
\psi: a_{(m)} \mapsto \pi\left(a t^{m}\right)
$$

para todos os geradores $a_{(m)}$ de $\mathfrak{g}, \operatorname{logo} \operatorname{Con}(\psi): R \rightarrow \operatorname{Con}(L / I, G / I)$ é dada por:

$$
\operatorname{Con}(\psi): a(z) \mapsto \operatorname{Con}(\pi)(a(z)), \forall a(z) \in R
$$

e é um isomorfismo por functorialidade. Seja $i:$ ConLieR $\rightarrow R$ o isomorfismo $\tilde{a}(z) \mapsto a(z)$ do teorema 3.20. Então $\operatorname{Con}(\psi)_{\circ} i=\operatorname{Con}(\pi) \mathrm{e}$ então $\operatorname{Con}(\pi)$ é um isomorfismo. Então o ideal $I$ é irregular por (3.10), o que demonstra o teorema.

Uma superálgebra de Lie de distribuições formais $(\mathfrak{g}, F)$ é chamada finita se $\bar{F}$ for um $\mathbb{C}[\partial]$-módulo finitamente gerado. $O$ teorema 3.21 reduz a classificação de superálgebras de Lie de distribuições formais finitas na classificação de superálgebras conformes (finitas), já que este teorema estabelece a equivalência entre a categoria das classes de equivalência das superálgebras de Lie de distribuições formais e a categoria das superálgebras conformes. Através do Corolário 3.9, a descrição das superálgebras conformes finitas recaem em dois problemas:

(1) descrever superálgebras conformes como sendo $\mathbb{C}[\partial]$-módulos livres de posto finito.

(2) encontrar extensões centrais de superálgebras conformes como em (1) com centro formado por elementos de torção.

Sendo assim, para classificar superálgebras conformes, basta lembrar que elas são soma direta de (1) e (2).

O primeiro problema a se resolver (analisar superálgebras conformes do tipo (1)) é reduzido à solução de um sistema finito de equações funcionais num conjunto finito de polinômios em duas indeterminadas como segue:

$$
\text { Seja } R=\bigoplus_{j=1}^{n} \mathbb{C}[\partial] a^{j} \text { um } \mathbb{C}[\partial] \text {-módulo } \mathbb{Z} / 2 \mathbb{Z} \text {-graduado com } p\left(a^{i}\right) \text { denotada }
$$


por $p(i)$ e seja $\left[a_{\lambda}^{i} a^{j}\right]=\sum_{k} Q_{k}^{i j}(\lambda, \partial) a^{k}$, onde $Q_{k}^{i j}$ são polinômios em $\lambda$ e $\partial$, para todo $i, j, k=1, \ldots, n$.

Este $\lambda$-colchete fornece uma estrutura de superálgebra conforme em $R$ se os $Q_{k}^{i j}$ estão subordinados às seguintes relações:

$$
\begin{gathered}
Q_{k}^{i j}(\lambda, \partial)=-(-1)^{p(i) p(j)} Q_{k}^{j i}(-\lambda-\partial, \partial) \\
\sum_{s=1}^{n}\left(Q_{s}^{j k}(\mu, \partial+\lambda) Q_{t}^{i s}(\lambda, \partial)-(-1)^{p(i) p(j)} Q_{s}^{i k}(\lambda, \partial+\mu) \cdot Q_{t}^{j s}(\mu, \partial)\right) \\
=\sum_{s=1}^{n} Q_{t}^{s k}(\lambda+\mu, \partial)
\end{gathered}
$$

Tem-se então o seguinte:

Proposição 3.22. As relações (3.11) e (3.12) são equivalentes respectivamente a $\left(C_{3}\right)_{\lambda}$ e a $\left(C_{4}\right)_{\lambda}$.

Demonstração. Provaremos a primeira equivalência, já que a segunda segue a mesma linha de raciocínio. Temos:

$$
\begin{aligned}
\Longrightarrow\left[a_{\lambda}^{i} a^{j}\right] & =\sum_{k=1}^{n} Q_{k}^{i j}(\lambda, \partial) a^{k} \\
& =-(-1)^{p(i) p(j)} \sum_{k} Q_{k}^{j i}(-\lambda-\partial, \partial) a^{k} \\
& =-(-1)^{p(i) p(j)}\left[a_{-\lambda-\partial}^{j} a^{i}\right]
\end{aligned}
$$

Logo vale $\left(C_{3}\right)_{\lambda}$.

$$
\begin{aligned}
& \Longleftarrow\left[a_{\lambda}^{i} a^{j}\right]=-(-1)^{p(i) p(j)}\left[a_{-\lambda-\partial}^{j} a^{i}\right] \Rightarrow \\
& \Rightarrow \sum_{k} Q_{k}^{i j}(\lambda, \partial) a^{k}=\sum_{k}-(-1)^{p(i) p(j)} Q_{k}^{j i}(-\lambda-\partial, \partial) a^{k}
\end{aligned}
$$

Como cada monômio em $a^{k}$ precisa ser igual para que as séries sejam iguais, então $Q_{k}^{i j}(\lambda, \partial)=-(-1)^{p(i) p(j)} Q_{k}^{j i}(-\lambda-\partial, \partial)$. Logo vale (3.11). 
É claramente impossível resolver diretamente estas equações para $n \geq 2$, mas para $n=1$ e $R=R_{0}$, será apresentada a seguinte solução: seja $R=[\partial] a$ e $\left[a_{\lambda} a\right]=Q(\lambda, \partial) a$, onde $Q(\lambda, \partial)$ é um polinômio em $\lambda$ e $\partial$ satisfazendo as duas equações abaixo:

$$
\begin{gathered}
Q(\lambda, \partial)=-Q(-\partial-\lambda, \partial) \\
Q(\mu, \partial+\lambda) \cdot Q(\lambda, \partial)-Q(\lambda, \partial+\mu) \cdot Q(\mu, \partial)=Q(\lambda,-\lambda-\mu) \cdot Q(\lambda+\mu, \partial)
\end{gathered}
$$

Seja $Q(\lambda, \partial)=\sum_{j=0}^{r} c \frac{1}{2}(\lambda) \partial^{j}$, com $c_{r}(\lambda) \neq 0$. Então comparando os coeficientes de $\partial^{2 r-1}$ em (3.14), obtem-se:

$r(\lambda-\partial) c_{r}(\lambda) c_{r}(\mu)=0$

se $r>1$, o que é um absurdo. De fato,

$$
Q(\mu, \partial+\lambda) Q(\lambda, \partial)=\sum_{j=0}^{r} c_{j}(\lambda)(\partial+\mu)^{j} \sum_{i=0}^{r} c_{i}(\mu) \partial^{i}
$$

Logo o termo em $\partial^{2 r-1}$ será:

$$
\begin{gathered}
c_{r}(\mu)\left[\partial^{r} c_{r-1}(\lambda) \partial^{r-1}+\lambda \cdot \partial^{r-1} c_{r}(\lambda) \partial^{r}\right]+c_{r-1}(\mu) \partial^{r-1} c_{r}(\lambda) \partial^{r} \\
=\left[c_{r}(\mu) c_{r-1}(\lambda)+\lambda \cdot c_{r}(\mu) c_{r}(\lambda)+c_{r}(\lambda) c_{r-1}(\mu)\right] \partial^{2 r-1}
\end{gathered}
$$

Agora:

$$
Q(\lambda, \partial+\mu) Q(\mu, \partial)=\sum_{j=0}^{r} c_{j}(\lambda)(\partial+\mu)^{j} \sum_{i=0}^{r} c_{i}(\mu) \partial^{i}
$$

Logo o termo em $\partial^{2 r-1}$ será:

$$
\begin{aligned}
& c_{r}(\lambda)\left[\partial^{r} c_{r-1}(\mu) \partial^{r-1}+\mu \cdot \partial^{r-1} c_{r}(\mu) \partial^{r}\right]+c_{r-1}(\lambda) \partial^{r-1} c_{r}(\mu) \partial^{r} \\
& =\left[c_{r}(\lambda) c_{r-1}(\mu)+\mu \cdot c_{r}(\lambda) c_{r}(\mu)+c_{r-1}(\lambda) \partial^{r-1} c_{r}(\mu) \partial^{r}\right. \\
& =\left[c_{r}(\lambda) c_{r-1}(\mu)+\mu \cdot c_{r}(\lambda) c_{r}(\mu)+c_{r-1}(\lambda) c_{r}(\mu)\right] \partial^{2 r-1}
\end{aligned}
$$


Finalmente:

$Q(\lambda,-\lambda-\mu) Q(\lambda+\mu, \partial)=Q(\lambda,-\lambda-\mu) \cdot \sum_{i=0}^{r} c_{i}(\lambda+\mu) \partial^{i}$

implica que $r$ é a maior potência de $\partial$, já que $Q(\lambda,-\lambda-\mu)$ não possui $\partial$, logo o termo em $\partial^{2 r-1}$ será nulo se $r>1$. Assim, se $r>1$, segue de (3.14) que:

$$
\begin{aligned}
& {\left[c_{r}(\mu) c_{r-1}(\lambda)+\lambda \cdot c_{r}(\mu) c_{r}(\lambda)+c_{r}(\lambda) c_{r-1}(\mu)\right]-} \\
& -\left[c_{r}(\lambda) c_{r-1}(\mu)+\mu \cdot c_{r}(\lambda) c_{r}(\mu)+c_{r-1}(\lambda) c_{r}(\mu)\right]=0
\end{aligned}
$$

Logo $(\lambda-\mu) c_{r}(\lambda) c_{r}(\mu)=0$ implica que $c_{r}(\lambda)=0$ ou $c_{r}(\mu)=0$ implica que $c_{r}(\lambda)=c_{r}(\mu)=0$, mas $c_{r}(\lambda) \neq 0$, por hipótese. Então:

$$
Q(\lambda, \partial)=a(\lambda) \partial+b(\lambda)
$$

Tomando $\lambda=\mu$ em (3.14), seguirá que

$$
Q(\lambda,-2 \lambda) Q(2 \lambda, \partial)=0
$$

Logo:

Caso 1: $Q(\lambda,-2 \lambda)=0$

Desta forma, como $Q(\lambda, \partial)=a(\lambda) \partial+b(\lambda)$, então:

$$
Q(\lambda,-2 \lambda)=a(\lambda) \cdot(-2 \lambda)+b(\lambda)
$$

Logo, $Q(\lambda,-2 \lambda)=0$ implica que $b(\lambda)=2 \lambda a(\lambda)$. Sendo assim,

$$
\begin{aligned}
Q(\lambda, \partial) & =a(\lambda) \partial+b(\lambda) \\
& =a(\lambda) \partial+2 \lambda a(\lambda) \\
& =a(\lambda)(\partial+2 \lambda)
\end{aligned}
$$

Aplicando isto à (3.13), tem-se que $a(\lambda)$ é uma constante, pois:

$$
\begin{aligned}
Q(\lambda, \partial) & =-Q(-\partial-\lambda, \partial) \\
& =-a(-\partial-\lambda)(\partial-2 \lambda) \\
& =a(-\partial-\lambda) \cdot(\partial+2 \lambda)
\end{aligned}
$$


Logo $a(\lambda)=a(-\partial-\lambda)$. Assim, vale que $a(\lambda)$ é constante, pois caso tivesse uma potência não-nula de $\lambda$, então $a(-\lambda-\partial)$ teria potência não-nula de $(-\lambda-\partial)$, $\operatorname{logo}$ de $\partial$, mas isto não ocorre, pois $a(\lambda)$ não possui potência não-nula de $\partial$. Logo $a(\lambda)$ é, de fato, constante. Assim:

$$
Q(\lambda, \partial)=a(2 \lambda+\partial)
$$

Caso 2: $Q(2 \lambda, \partial)=0$.

Assim, $Q(\lambda, \partial)=0$, bastando trocar $\lambda$ por $\frac{\lambda}{2}$. Desta forma, conseguimos no primeiro caso uma álgebra conforme comutativa (todos os produtos são triviais) e no segundo caso, conseguimos a álgebra conforme de Virasoro, a qual será estudada com mais detalhes adiante.

Agora será discutido brevemente o segundo problema, o da construção de extensões centrais $\hat{R}=R \oplus C$, onde $R$ e $C$ são $\mathbb{C}[\partial]$-submódulos de $\hat{R}$ e $c_{\lambda} \hat{R}=0$. O $\lambda$-colchete:

$$
\left[a_{\lambda} b\right]^{\wedge}=\left[a_{\lambda} b\right]+\alpha_{\lambda}(a, b)
$$

onde $\left[a_{\lambda} b\right]$ é o $\lambda$-produto em $R$ e:

$$
\alpha_{\lambda}(a, b)=\sum_{n \geq 0} \lambda^{(n)} \alpha_{n}(a, b)
$$

é uma aplicação $\mathbb{C}$-linear de $R \otimes R$ em $\mathbb{C}[\lambda] \underset{\mathbb{C}}{\otimes} C$.

Os axiomas $\left(C_{2}\right)_{\lambda},\left(C_{2}^{\prime}\right)_{\lambda},\left(C_{3}\right)_{\lambda}$ e $\left(C_{4}\right)_{\lambda}$ para $\hat{R}$ são equivalentes às seguintes propriedades do 2-cociclo $\alpha_{\lambda}(a, b)$ :

$$
\begin{gathered}
\alpha_{\lambda}(\partial a, b)=-\lambda \cdot \alpha_{\lambda}(a, b) \\
\alpha_{\lambda}(a, \partial b)=(\partial+\lambda) \alpha_{\lambda}(a, b) \\
\alpha_{\lambda}(a, b)=-(-1)^{p(a) p(b)} \alpha_{-\lambda-\partial}(b, a) \\
\alpha_{\lambda}\left(a, b_{\mu} c\right)-(-1)^{p(a) p(b)} \alpha_{\mu}\left(b, a_{\lambda} c\right)=\alpha_{\lambda+\mu}\left(a_{\lambda} b, c\right)
\end{gathered}
$$


De fato, (3.16) é equivalente a $\left(C_{2}\right)_{\lambda}$ pois $\left[\partial a_{\lambda} b\right]^{\wedge}=\left[\partial a_{\lambda} b\right]+\alpha_{\lambda}(\partial a, b)$ e:

$$
\begin{aligned}
-\lambda\left[a_{\lambda} b\right]^{\wedge} & =-\lambda\left(\left[a_{\lambda} b\right]+\alpha_{\lambda}(a, b)\right) \\
& =-\lambda\left[a_{\lambda} b\right]-\lambda \alpha_{\lambda}(a, b) \\
& =\left[\partial a_{\lambda} b\right]-\lambda \alpha_{\lambda}(a, b)
\end{aligned}
$$

já que $R$ é conforme. Logo $\left[\partial a_{\lambda} b\right]^{\wedge}=-\lambda\left[a_{\lambda} b\right]^{\wedge}$ se e somente se $\alpha_{\lambda}(\partial a, b)=$ $-\lambda \alpha_{\lambda}(a, b)$ e assim, segue que (3.16) é equivalente à $\left(C_{2}\right)_{\lambda}$. As demais equivalências seguem de forma análoga.

Como antes, estas equações são equivalentes a um sistema de equações funcionais em um conjunto de polinômios de duas indeterminadas. Agora serão apresentados três importantes exemplos de superálgebras conformes finitas $R$. Através de $\left(C_{2}\right)$ e de $\left(C_{2}^{\prime}\right)$, é suficiente definir os $n$-ésimos produtos nos geradores do $\mathbb{C}[\partial]$-módulo $R$.

Exemplo 3.23. Seja $\mathfrak{g}$ uma superálgebra de Lie de dimensão finita. Temos:

$$
[a(z), b(w)]=[a, b](w) \delta(z-w)
$$

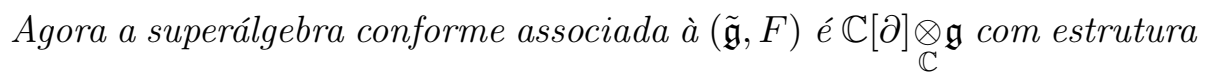
de superálgebra conforme definida em $a, b \in \mathfrak{g}$ por:

$$
\begin{aligned}
& a_{(0)} b=[a, b] \\
& a_{(m)} b=0, \forall m \geq 1
\end{aligned}
$$

Esta é chamada de superálgebra conforme de Corrente associada a $\mathfrak{g} e$ é denotada por Curg. Agora tais fórmulas definem um 2-cociclo em Curg com valores no $\mathbb{C}[\partial]$-módulo trivial $\mathbb{C}$

$$
\begin{aligned}
& \alpha_{1}(a, b)=(a \mid b) \\
& \alpha_{m}(a, b)=0, \text { se } m \neq 1
\end{aligned}
$$

onde $(\cdot \mid \cdot)$ é uma forma bilinear supersimétrica invariante em $\mathfrak{g}$. Pode-se ver que (3.22) fornece todos os 2-cociclos, tomando para $\alpha_{0}$ um 2-cociclo em $\mathfrak{g} e$ 
provando que $[\mathfrak{g}, \mathfrak{g}]=\mathfrak{g}$.

Em particular, se $\mathfrak{g}$ é uma álgebra de Lie simples de dimensão finita, então (3.22) fornece todos os 2-cociclos por meio de equivalências. A correspondente extensão central é a superálgebra conforme Con $\mathfrak{\mathfrak { g }}$ associada à álgebra de Corrente $\hat{\mathfrak{g}}$ definida no Capítulo 2. Segue do Teorema 3.14 que $\operatorname{Lie}(\operatorname{Con} \hat{\mathfrak{g}})=\mathfrak{g}$ e que $\operatorname{Lie}(\operatorname{Cur} \mathfrak{g})=\tilde{\mathfrak{g}}$, isto é, tanto $\tilde{\mathfrak{g}}$ quanto $\hat{\mathfrak{g}}$ são superálgebras de Lie de distribuições formais maximais. Note que $I=\mathfrak{g}\left[t, t^{-1}\right] \cdot p(t)$, onde $p(t)$ é um polinômio de Laurent não-inversível, é um ideal irregular de $\tilde{\mathfrak{g}}$, onde as superálgebras de Lie de distribuições formais $\tilde{\mathfrak{g}}$ e $\tilde{\mathfrak{g}} / I$ geram a mesma superálgebra conforme.

Exemplo 3.24. Denotemos Vect $\mathbb{C}^{\times}$a álgebra de Lie dos campos vetoriais regulares em $C^{\times}$. Ela tem uma base $L_{n}=-t^{n+1} \partial_{t}$ (onde $\in \mathbb{Z}$ ) com a seguinte relação de comutação:

$$
\left[L_{m}, L_{n}\right]=(m-n) L_{m+n}
$$

Esta é uma superálgebra de Lie de distribuições formais com a família $F$ consistindo de uma distribuição formal simples:

$$
L(z)=\sum n \cdot L_{n} z^{-n-2}=-\delta(z-t) \partial_{t}
$$

Disto segue que:

$$
[L(z), L(w)]=\partial_{w} L(w) \delta(z-w)+2 L(w) \partial_{w} \delta(z-w)
$$

Então a álgebra conforme associada à $\left(V e c t C^{\times}, L(z)\right)$ é Con $\left(V e c t C^{\times}\right)=$ $\mathbb{C}[\partial] L$, com o produto:

$$
\begin{aligned}
& L_{(0)} L=\partial L \\
& L_{(1)} L=2 L \\
& L_{(m)} L=0, \text { se } m \geq 2
\end{aligned}
$$

Esta é a chamada álgebra conforme de Virasoro (e é denotada por Vir) já vista anteriormente em termos do $\lambda$-colchete $\left[L_{\lambda} L\right]=(\partial+2 \lambda) L$. Pode-se 
observar que esta álgebra conforme possui um único, a menos de equivalência, 2-cociclo, dado por:

$$
\begin{aligned}
& \alpha_{3}(L, L)=\frac{c}{2} \\
& \alpha_{m}(L, L)=0, \text { se } m \neq 3
\end{aligned}
$$

A extensão central correspondente é a álgebra conforme Con(Vir) associada à álgebra de Virasoro. Note que ambos, Vect $\mathbb{C}^{\times}$e Vir, são álgebras de Lie de distribuições formais maximais e ambos não possuem ideais irregulares.

Exemplo 3.25. A soma semidireta $\left(\right.$ Vect $\left.C^{\times}\right)+\tilde{\mathfrak{g}}$ definida por:

$$
\left[f(t) \partial_{t}, a \otimes \mathfrak{g}(t)\right]=a \otimes f(t) \partial_{t} g(t)
$$

é uma álgebra de Lie de distribuições formais maximais sem ideais irregulares. Tem-se que:

$$
[L(z), L(w)]=\left(\partial_{w} a(w)\right) \delta(z-w)
$$

Então a álgebra associada a $\left(\right.$ Vect $\left.C^{\times}\right)+\tilde{\mathfrak{g}}$ é a soma semidireta

$\operatorname{Conf}\left(\operatorname{Vect} C^{\times}\right)+$Cur $\mathfrak{g}$, definida por $(a \in \mathfrak{g})$ :

$$
\begin{aligned}
& L_{(0)} a=\partial a ; \\
& L_{(1)} a=a ; \\
& L_{(m)} a=0, \text { para } m>1
\end{aligned}
$$

Agora diz-se que uma superálgebra conforme é simples se ela for comutativa e não contiver ideais não-triviais. Tem-se o seguinte resultado:

Afirmação 3.26. ([DK]) Toda álgebra conforme simples e finita é isomorfa ou a uma álgebra conforme de Corrente Curg, onde $\mathfrak{g}$ é uma álgebra de Lie simples de dimensão finita, ou a uma álgebra conforme de Virasoro. 
A demonstração desta afirmação é bastante extensa e não será feita neste trabalho, mas ela mostra a importância do estudo destas duas álgebras e como tudo se relaciona. Agora traduzindo a afirmação para a linguagem das álgebras de Lie de distribuições formais, obtem-se o seguinte resultado:

"Toda álgebra de Lie de distribuições formais finita e simples é isomorfa ou a $\left(V e c t C^{\times}, L(s)\right)$ ou ao quociente de $(\tilde{\mathfrak{g}},\{a(z) / a \in \mathfrak{g}\})$, onde $\mathfrak{g}$ é uma álgebra de Lie simples de dimensão finita".

Definição 3.27. Defina álgebra derivada de $R$ e a denote por $R^{\prime}$ como sendo o $\mathbb{C}$-gerado de todos os elementos da forma $a_{(m)}$ b de uma superálgebra conforme $R$, com $m \in \mathbb{Z}_{+}$.

Pode-se ver que $R^{\prime}$ é um ideal de $R$ tal que $R / R^{\prime}$ é comutativo. Agora tem-se a série derivada

$$
R \supset R^{\prime} \supset R^{\prime \prime} \supset \ldots
$$

Definição 3.28. Uma superálgebra conforme é chamada solúvel se o n-ésimo membro da série (3.28) for nulo para $n \gg 0$, e é chamada semisimples se não contiver ideais solúveis não-nulos.

Um dos resultados mais importantes de $[D K]$ diz que toda álgebra conforme semisimples finita é uma soma direta de álgebras conformes dos seguintes tipos:

(i) álgebras conformes de Correntes $C u r \mathfrak{g}$, onde $\mathfrak{g}$ é uma álgebra de Lie semisimples de dimensão finita.

(ii) álgebras conformes de Virasoro.

(iii) a soma semidireta de (i) e de (ii).

\section{3 Álgebra de Vertex}

De acordo com os princípios gerais da teoria dos campos quânticos, as observáveis são campos quânticos que são operadores de distribuições valuadas no espaço-tempo, satisfazendo certos axiomas, chamados de axiomas de Wightman (ver Capítulo 1 de [5] para maiores detalhes). 
Os dados de uma álgebra de Vertex consistem de um espaço de estado $V$ (espaço vetorial arbitrário), de um vetor vácuo $\mid 0>\in V$, de um operador de translação infinitesimal $T \in$ End $V$ e de uma coleção de campos quânticos End $V$-valuados $\mathfrak{F}$, sujeitos aos axiomas abaixo:

(vácuo) $T \mid 0>=0$,

(translação covariante) $\left[T, a^{j}(z)\right]=\partial_{z} a^{j}(z)$,

(localidade) $(z-w)^{N_{j_{k}}}\left[a^{j}(z), a^{k}(w)\right]=0$, para algum $N_{j_{k}} \in \mathbb{Z}_{+}$,

(completude) Os vetores $a_{\left(n_{1}\right)}^{j_{1}} \ldots a_{\left(n_{s}\right)}^{j_{s}} \mid 0>$ geram $V$.

Observação: Um campo quântico $a(z)$ é uma distribuição formal $E n d V$ valuada, que é uma função linear End $V$-valuada no espaço dos polinômios de Laurent $\mathbb{C}\left[z, z^{-1}\right]$ (o espaço das funções de teste), que satisfazem a condição de $a(z) v$ ser uma série de Laurent em $z$, para todo $v \in V$. Pode-se escrever $a(z)$ na forma:

$a(z)=\sum_{n \in \mathbb{Z}} a_{(n)} z^{-n-1}$

onde $a_{(n)} \in$ End $V$ é tal que $a_{(n)}=\operatorname{Res}_{z} z^{n} a(z)$. Esta condição diz que $a_{(n)} v=0$, para $n \gg 0$.

Agora observe que estendendo $\mathfrak{F}$ para uma coleção maximal $\overline{\mathfrak{F}}$ de campos quânticos nos quais os axiomas valem, então a aplicação $\overline{\mathfrak{F}} \rightarrow V$ definida por $a(z) \mapsto a_{(-1)} \mid 0>$ é bijetora. Então tem-se a correspondência de estadocampo, definida como a aplicação inversa $V \rightarrow \overline{\mathfrak{F}}$, onde

$$
V \ni a \mapsto Y(a, z) \in \overline{\mathfrak{F}}
$$

que leva a uma segunda definição de álgebra de Vertex. Será usada a notação $Y(a, z)$ dos campos quânticos, correspondente ao estado $a \in V$ e será usado para este campo quântico o nome operador de Vertex (em inglês, Vertex Operator). A correspondência estado-campo permite introduzir um produto bilinear em $V$, para todo $n \in \mathbb{Z}$, fazendo

$$
a_{(n)} b=\operatorname{Res}_{z} z^{n} Y(a, z) b
$$

Isto permite uma terceira definição de álgebra de Vertex como um espaço vetorial $V$ com o vetor vácuo $\mid 0>$ e produto bilinear $a_{(n)} b$, para todo $n \in \mathbb{Z}$, 
satisfazendo uma simples propriedade chamada vácuo e uma bem mais complexa, chamada identidade de Borcherds.

Para finalizar, a quarta definição de álgebra de Vertex será, neste contexto, a mais importante e que vale a pena frisar, pois permite visualizar uma álgebra de Vertex como uma superálgebra conforme. O objetivo não é desenvolver esta teoria, mas sim ilustrar a relação existente entre estas, mostrando o quanto o conhecimento já desenvolvido sobre superálgebras conformes poderá ser útil no estudo de Álgebras de Vertex, uma vez que a estrutura de superálgebra conforme está imbutida nas outras três definições (devido à equivalência destas). Para finalizar, segue as quatro definições de Álgebras de Vertex:

Definição 3.29. Uma álgebra de Vertex é uma quádrupla $(V, \mid 0>, T, \mathfrak{F})$, onde $V=V_{(\overline{0})} \oplus V_{(\overline{1})}$ é um superespaço vetorial, chamado de espaço de estados, $\mid 0>$ é um elemento de $V_{\overline{0}}$, chamado de vetor vácuo, $T$ é um endomorfismo par de $V$, chamado de operador de translação infinitesimal, e $\mathfrak{F}$ é uma coleção de campos quânticos End V-valuados, isto é,

$$
\mathfrak{F}=\left\{a^{j}(z)=\sum_{n \in \mathbb{Z}} a_{(n)}^{j} z^{-n-1}\right\}_{j \in J}
$$

onde para cada $j \in J$, todo $a_{(n)}^{j} \in$ End $V$ possue a mesma paridade e, para todo $b \in V$, temos que $a_{(n)}^{j} b=0$, para $n \gg 0$.

O enunciado acima satisfaz os seguintes axiomas:

(i) vácuo: $T \mid 0>=0$

(ii) translação covariante: $\left[T, a^{j}(z)\right]=\partial_{z} a^{j}(z)$

(iii) localidade: $(z-w)^{N_{j_{k}}}\left[a^{j}(z), a^{k}(w)\right]=0$, para algum $N_{j_{k}} \in \mathbb{Z}_{+}$.

(iv) completude: $V=\operatorname{span}_{\mathbb{C}}\left\{a_{\left(n_{1}\right)}^{j_{1}} \ldots a_{\left(n_{s}\right)}^{j_{s}} \mid 0>\right\}$,

onde o comutador é dado por:

$$
[a, b]=a b-p(a, b) b a
$$

$\operatorname{com} p(a, b)=(-1)^{p(a) p(b)}$.

A segunda definição, bastante utilizada em [1] é dada por:

Definição 3.30. Uma álgebra de Vertex é uma tripla $(V, \mid 0>, Y)$, onde $V=V_{\overline{0}} \oplus V_{\overline{1}}$ é um superespaço vetorial (o espaço de estados), $\mid 0>\in V_{\overline{0}}$ é um 
vetor (o vetor vácuo), e $Y$ é uma aplicação linear que preserva paridade de $V$ para o espaço dos campos quânticos End $V$-valuados: $Y(a, z)=$ $\sum_{n \in \mathbb{Z}} a_{(n)} z^{-n-1}$, chamado o estado de campo correspondente. O operador de translação infinitesimal $T$ é dado no estado de campo correspondente pela fórmula:

$$
T a=a_{(-2)} \mid 0>
$$

Os axiomas são os seguintes:

(i) vácuo: $T|0>=0, Y(a, z)| 0>\left.\right|_{z=0}=a$,

(ii) translação covariante: $[T, Y(a, z)]=\partial_{z} Y(a, z)$,

(iii) localidade: $(z-w)^{N}[Y(a, z), Y(b, w)]=0$, para algum $N \in \mathbb{Z}_{+}$.

Já a terceira definição é:

Definição 3.31. Uma álgebra de Vertex é uma tripla $(V, \mid 0>, Y)$, onde $V$ e $\mid 0>$ são como na segunda definição e $Y$ é uma correspondência estadocampo injetiva $a \mapsto Y(a, z)$ tal que :

(i) $Y(\mid 0>, z)=I_{V}$

(ii) $Y(a, z) Y(b, w) i_{z, w}(z-w)^{n}-p(a, b) Y(b, w) Y(a, z) i_{w, z}(z-w)^{n}$

$=\sum_{j \in \mathbb{Z}_{+}} Y\left(a_{(n+j)} b, w\right) \partial_{w}^{j)} \delta(z-w)$

para todo $n \in \mathbb{Z}$ e $a, b \in V$.

Observe que a soma a direita é finita, já que $a_{(N)} b=0$, para $N \gg 0$, uma vez que $Y(a, z)$ é um campo quântico. Definindo o produto ordenado normal em $V$ por:

$$
: a b:=a_{(-1)} b=\operatorname{Res}_{z} z^{-1} Y(a, z) b
$$

temos a última definição (e neste trabalho, a mais importante):

Definição 3.32. Uma álgebra de Vertex é uma quíntupla $\left(V, \mid 0>, T,\left[\cdot \lambda^{\cdot}\right],::\right)$, onde: 
(i) $\left(V, T,\left[\cdot_{\lambda} \cdot\right]\right)$ é uma superálgebra de Lie conforme

(ii) $(V, \mid 0>, T,::)$ é uma superálgebra diferencial (isto é, $T$ é uma derivação) unitária, satisfazendo:

$$
\begin{gathered}
: a b:-p(a, b): b a:=\int_{-T}^{0}\left[a_{\lambda} b\right] d \lambda \\
:: a b: c:-: a: b c::=:\left(\int_{0}^{T} d \lambda a\right)\left[b_{\lambda} c\right]:+p(a, b):\left(\int_{0}^{T} d \lambda b\right)\left[a_{\lambda} c\right]:
\end{gathered}
$$

(iii) o $\lambda$-colchete $[\cdot \lambda \cdot]$ e o produto :: estão relacionados pela (não-comutativa) fórmula de Wick abaixo:

$$
\left[a_{\lambda}: b c:\right]=:\left[a_{\lambda} b\right] c:+p(a, b): b\left[a_{\lambda} c\right]:+\int_{0}^{\lambda}\left[\left[a_{\lambda} b\right]_{\mu} c\right] d \mu
$$




\section{Capítulo 4}

\section{Módulos Conformes}

O objetivo principal deste Capítulo é classificar os módulos conformes finitos e irredutíveis. Assim, serão apresentado até o Teorema 4.8 as definições, propriedades e os exemplos básicos que permitirão iniciar este estudo. Em seguida, apresenta-se a construção de LieR e a utilidade da $\lambda$-ação no contexto dos módulos conformes, todavia não serão feitas as demonstrações, já que tais assuntos possuem seus análogos abordados no Capítulo 3. Por exemplo, constrói-se um funtor da categoria dos LieRmódulos conformes para a categoria dos $R$-módulos conformes em que se estabelece a "equivalência" (no mesmo sentido que o do Capítulo 3) entre ambas que permite classificar os módulos conformes finitos. Devemos destacar a importância dos exemplos 4.13, 4.15, 4.16 e 4.17, em que são apresentados os módulos referentes aos teoremas principais. O Teorema 4.18 fornece um resultado bastante útil, já que afirma que todo módulo conforme finito, irredutível e não-trivial sobre a álgebra de Virasoro é isomorfo ao módulo $M_{\beta}^{c}(\alpha, \Delta)$ do exemplo 4.13 , onde $\Delta \in \mathbb{C}^{*}$.

Já na Seção 4.2, apresenta-se a definição de $\mathfrak{L}$-módulo conforme e um lema auxiliar. Assim, define-se o módulo induzido $\operatorname{Ind}_{\mathfrak{L}_{0}}^{\mathfrak{L}} V=U(\mathfrak{L}) \underset{U\left(\mathfrak{L}_{0}\right)}{\otimes} V$ e prova-se o Teorema 4.21 e também o Lema 4.22, dois dos resultados mais sofisticados deste trabalho. Como consequiência deles, tem-se os Corolários de 4.23 a 4.28 , cujos enunciados terão muita utilidade posteriormente. Depois faz-se uma observação que invalidava o resultado provado em [3], consertada posteriormente através de uma errata. Feito isto, o trabalho estará terminado.

É importante ressaltar que muito do que foi feito aqui tem o seu análogo no Capítulo 3. Por isso, uma leitura preliminar desse é imprescindível. 


\subsection{Introdução}

Definição 4.1. Seja $(\mathfrak{g}, F)$ uma superálgebra de Lie de distribuições formais e seja $V$ um $\mathfrak{g}$-módulo. Diz-se que uma distribuição formal $a(z) \in F$ e uma distribuição formal $v(z)=\sum_{n \in \mathbb{Z}} v_{(n)} z^{-n-1}$ com coeficientes em $V$ são locais se:

$$
(z-w)^{N} a(z) v(w)=0
$$

para algum $N \in \mathbb{Z}_{+}$.

Segue do Capítulo 2 que (4.1) é equivalente à:

$$
a(z) v(w)=\sum_{j=0}^{N-1}\left(a_{(j)} v\right)(w) \partial_{w}^{(j)} \delta(z-w)
$$

para algumas distribuições formais $\left(a_{(j)} v\right)(w)$, com coeficientes em $V$, que são univocamente determinados pelas fórmulas:

$$
\left(a_{(j)} v\right)(w)=\operatorname{Res}_{z}(z-w)^{j} a(z) v(w)
$$

lembrando que todo elemento $a(z, w)$ que satisfaz $(z-w)^{N} a(z, w)=0$ para $N \gg 0$ é da forma:

$$
a(z, w)=\sum_{j=0}^{N-1} c^{j}(w) \partial_{w}^{(j)} \delta(z-w)
$$

onde $c^{j}(w)=\operatorname{Res}_{z} a(z, w)(z-w)^{j}$ e assim, basta definir $a(z, w)=a(z) v(w)$ que (4.2) segue (ver Seção 2). Esta fórmula é, então, equivalente a:

$$
a_{(m)} v_{(n)}=\sum_{j \in \mathbb{Z}_{+}}\left(\begin{array}{c}
m \\
j
\end{array}\right)\left(a_{(j)} v\right)_{(m+n-j)}
$$

Exemplo 4.2. Considere a seguinte representação da álgebra de Virasoro no espaço vetorial $V$ com elementos de base $v_{(n)}$, com $n \in \mathbb{Z}$, sobre $\mathbb{C}$ :

$L_{m} v_{(n)}=((\Delta-1)(m+1)-n) v_{(m+n)}+\alpha v_{(m+n+1)}$, onde $\Delta, \alpha \in \mathbb{C}$. 
Em termos das superálgebras de Lie de distribuições formais $L(z)$ e v(z), isto pode ser reescrito como:

$$
L(z) v(w)=(\partial+\alpha) v(w) \delta(z-w)+\Delta v(w) \partial_{w} \delta(z-w)
$$

Portanto, $L(z)$ e v(z) são locais.

Definição 4.3. Seja $(\mathfrak{g}, F)$ uma distribuição formal de superálgebras de Lie e seja $V$ um $\mathfrak{g}$-módulo gerado sobre $\mathbb{C}$ pelos coeficientes de uma família $E$ de distribuiçôes formais tais que todos os pares $(a(z), v(z))$, onde $a(z) \in F$ e $v(z) \in E$ são locais. Então $(V, E)$ é chamado de módulo conforme sobre $(\mathfrak{g}, F)$.

O Lema seguinte é análogo ao Lema de Dong, provado na Seção 3.2 de [1].

Lema 4.4. Seja $V$ um módulo sobre uma superálgebra de Lie $\mathfrak{g}$, seja $a(z), b(z) \in \mathfrak{g}\left[\left[z, z^{-1}\right]\right]$ e $v(z) \in V\left[\left[z, z^{-1}\right]\right]$.

(a) Se $(a(z), v(z))$ é um par local, então os dois pares $(\partial a(z), v(z))$ e $(a(z), \partial v(z))$ são locais.

(b) Se todos os três pares $(a(z), b(z)),(a(z), v(z))$ e $(b(z), v(z))$ são locais, então os pares $\left(a(z)_{(j)} b(z), v(z)\right)$ e $\left(a(z), b(z)_{(j)} v(z)\right)$ são locais, para todo $j \in \mathbb{Z}_{+}$.

Demonstração. Sabemos que $(z-w)^{N} a(z) v(w)=0$ para $N \gg 0$. Logo:

$(z-w)^{N+1} a(z) v(w)=0$

$\partial_{z}\left((z-w)^{N+1} a(z) v(w)\right)=0$

$(N+1) \cdot(z-w)^{N} a(z) v(w)+(z-w)^{N+1} \partial_{z} a(z) v(w)=0$

Logo $(z-w)^{N+1} \partial_{z} a(z) v(w)=0$, o que prova que $(\partial a(z), v(z))$ é local. A prova de que $(a(z), \partial v(z))$ é local segue o mesmo raciocínio. Para provar (b), observemos que $\exists N_{1}, N_{2}, N_{3} \in \mathbb{N}$ tais que:

$$
\begin{aligned}
& (z-w)^{N_{1}} a(z) b(w)=0 \\
& (z-w)^{N_{2}} a(z) v(w)=0
\end{aligned}
$$


$(z-w)^{N_{3}} b(z) v(w)=0$

Agora preciso mostrar que $\exists N \in \mathbb{N}$ tal que $(z-w)^{N} a(z)_{(j)} b(z) \cdot v(w)=0$, com $j \in \mathbb{Z}_{+}$fixado qualquer. De fato, seja $N=N_{3}$. Então:

$$
\begin{aligned}
(z-w)^{N} a(z)_{(j)} b(z) v(w) & =(z-w)^{N}\left(\operatorname{Res}_{x}(x-z)^{j} a(x) b(z)\right) v(w) \\
& =\operatorname{Res}_{x}(x-z)^{j} a(x)(z-w)^{N} b(z) v(w) \\
& =\operatorname{Res}_{x}(x-z)^{j} a(x) \cdot 0 \\
& =0
\end{aligned}
$$

Logo $\left(a(z)_{(j)} b(z), v(z)\right)$ é local, para todo $j \in \mathbb{Z}_{+}$. Analogamente, segue que $\left(a(z), b(z)_{(j)} v(z)\right)$ também é um par local.

Este lema mostra que a família $E$ de um módulo conforme $(V, E)$ sobre $(\mathfrak{g}, F)$ pode sempre ser incluída em seu fecho, isto é, na família minimal $\bar{E}$ (que contém $E$ ) que é local com respeito a $F$, e tal que $\mathbb{C}[\partial] \bar{E} \subset \bar{E}$ e $a_{(j)} \bar{E} \subset \bar{E}$, para todo $a \in F$ e $j \in \mathbb{Z}_{+}$. O mesmo lema mostra que $\bar{E}$ é local com respeito à $\bar{F}$. Assim, tem-se o seguinte corolário:

Corolário 4.5. (a) Se uma superálgebra de Lie gé gerada (como uma álgebra) pelos coeficientes de uma família de distribuições formais mutuamente locais sobre $F$, então $(\mathfrak{g}, \bar{F})$ é uma superálgebra de Lie de distribuições formais.

(b) Se $V$ é um módulo sobre uma superálgebra de Lie de distribuições formais $(\mathfrak{g}, F)$ gerada (como um módulo) pelos coeficientes de uma família $E$ de distribuições formais tais que todos os pares $(a(z), v(z))$, onde $a(z) \in F$ e $v(z) \in E$ são locais, então $(V, \bar{E})$ é um módulo conforme sobre $(\mathfrak{g}, \bar{F})$.

Agora os módulos conformes sobre uma superálgebra de Lie de distribuições formais $(\mathfrak{g}, F)$ formam uma categoria com morfismos $\varphi$ : $(V, E) \rightarrow\left(V_{1}, E_{1}\right)$ sendo homomorfismos de $\mathfrak{g}$-módulos $\varphi: V \rightarrow V_{1}$ tais que $\varphi(E) \subset E_{1}$, já que

$$
a(z)=\sum_{n} v_{(n)} z^{-n-1} \in E
$$

e também que

$$
\varphi(a(z))=\sum_{n} \varphi\left(v_{(n)}\right) z^{-n-1} \in E_{1}
$$


e que $v_{(n)} \in V$ e $\varphi\left(v_{(n)}\right) \in V_{1}$. Os mesmos cálculos feitos na demonstração do Teorema 2.12, ítens (iii) e (iv), mostram que:

$$
\begin{gathered}
{\left[a_{(m)}, b_{(n)}\right] v=\sum_{j=0}^{m}\left(\begin{array}{c}
m \\
j
\end{array}\right)\left(a_{(j)} b\right)_{(m+n-j)} v} \\
(\partial a)_{(n)} v=\left[\partial, a_{(n)}\right] v=-n \cdot a_{(n-1)} v
\end{gathered}
$$

onde $[\cdot, \cdot]$ é o colchete de operadores em $\bar{E}$.

Proposição 4.6. Para todo $a \in \bar{F}$ e $j \in \mathbb{Z}_{+}$, temos que $a_{(j)} \bar{E} \subset \bar{E}$.

Demonstração. Suponhamos que todo elemento de $\bar{F}$ fosse da forma $a(z)_{(j)} b(z)$ com $a(z), b(z) \in F$. Então precisamos mostrar que $\forall v(z) \in \bar{F}$, temos que $\left(a(z)_{(j)} b(z)\right)_{(n)} v(z) \in \bar{F}$. Agora $\left[a(w)_{(0)} b(w)_{(n)}\right] v(w) \in \bar{E}$, pois $F$ é fechado para o colchete, logo $\left[a(w)_{(0)} b(w)_{(n)}\right]=c(w)_{(k)} \in F$ e com $F$ funciona, mas:

$$
\begin{aligned}
{\left[a(w)_{(0)}, b(w)_{(n)}\right] v(w) } & =\sum_{j=0}^{0}\left(\begin{array}{l}
o \\
j
\end{array}\right)\left(a(w)_{(j)} b(w)\right)_{(0+n-j)} v(w) \\
& =\left(a(w)_{(0)} b(w)\right)_{(n)} v(w)
\end{aligned}
$$

Logo vale para $j=0$. Agora suponhamos válido para $j=m-1$ e provemos para $j=m$.

$$
\begin{aligned}
& \bar{E} \ni {\left[a(w)_{(m)}, b(w)_{(n)}\right] v(w)=\sum_{j=0}^{m}\left(\begin{array}{c}
m \\
j
\end{array}\right)\left(a(w)_{(j)} b(w)\right)_{(m+n-j)} v(w) } \\
&=\left(a(w)_{(m)} b(w)\right)_{(n)} v(w)+\sum_{j=0}^{m-1}\left(\begin{array}{c}
m \\
j
\end{array}\right)\left(a(w)_{(j)} b(w)\right)_{(m+n-j)} v(w)
\end{aligned}
$$

Para $0 \leq j \leq m-1$, temos que $\left(a(w)_{(j)} b(w)\right)_{(m+n-j)} v(w) \in \bar{E}$, por hipótese. Assim:

$$
\left(\begin{array}{c}
m \\
j
\end{array}\right)\left(a(w)_{(j)} b(w)\right)_{(m+n-j)} v(w) \in \bar{E}
$$

e, portanto:

$$
\left(a(w)_{(m)} b(w)\right)_{(n)} v(w) \in \bar{E}
$$


já que $\left[a(w)_{(m)}, b(w)_{(n)}\right] v(w) \in \bar{E}$. Assim mostramos que $a_{(j)} \bar{E} \subset \bar{E}$, para todo elemento $a \in \bar{F}$ da forma $a=a(z)_{(j)} b(z)$, com $a(z), b(z) \in F$. Para $a \in \bar{F}$ qualquer, bastará repetir este raciocínio quantas vezes for necessário, lembrando que $a$ é algo do tipo:

$$
a_{1\left(j_{1}\right)} a_{2\left(j_{2}\right)}\left(a_{3\left(j_{3}\right)} a_{4}\right)_{\left(j_{4}\right)} \ldots a_{n\left(j_{n}\right)} a_{(n+1)}
$$

onde $a_{1}, \ldots, a_{n+1} \in F$, pela definição de $\bar{F}$.

Assim, todo módulo conforme $(V, E)$ sobre uma superálgebra de Lie de distribuições formais $(\mathfrak{g}, F)$ é mandado para um módulo conforme $M(V)=\bar{E}$ sobre a superálgebra conforme $\bar{F}$, definido como:

Definição 4.7. Um módulo $M$ sobre uma superálgebra conforme $R$ é um $\mathbb{C}[\partial]$-módulo à esquerda, $\mathbb{Z} / 2 \mathbb{Z}$-graduado, com aplicaçôes $\mathbb{C}$-linear $a \mapsto a_{(n)}^{M}$ de $R$ em End $\mathbb{C}_{\mathbb{C}} M$, para todo $n \in \mathbb{Z}_{+}$, tais que as seguintes propriedades sejam satisfeitas, para $a, b \in R, v \in M$ e $m, n \in \mathbb{Z}_{+}$:

$\left(M_{1}\right)(\partial a)_{(n)}^{M} v=\left[\partial^{M}, a_{(n)}^{M}\right] v=-n \cdot a_{(n-1)}^{M} v ;$
$\left(M_{2}\right)\left[a_{(m)}^{M}, b_{(n)}^{M}\right] v=\sum_{j=0}^{m}\left(\begin{array}{c}m \\ j\end{array}\right)\left(a_{(j)} b\right)_{(m+n-j)}^{M} v$

Um R-módulo $M$ é dito conforme se satisfaz:

$\left(M_{0}\right) a_{(n)}^{M} v=0$, para $n \gg 0$.

Teorema 4.8. Seja $M$ um módulo arbitrário sobre uma superálgebra conforme $R$. Então $(\partial R)_{(0)} M=0$ e assim a aplicação $R_{(0)} M \rightarrow M$ induz em $M$ uma estrutura de módulo sobre a superálgebra de Lie $R / \partial R$ com relação

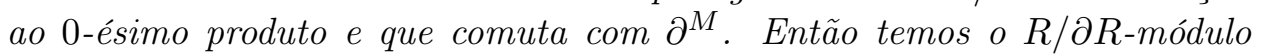
$M / \partial^{M} M$.

Demonstração. $M$ é um $R$-módulo, $R_{(0)} M \stackrel{\varphi}{\rightarrow} M$. Agora $r \circ m=r_{(0)} m$. Logo $(\partial r) \circ m=(\partial r)_{(0)} m=0$, o que implica que $\partial R \subset \operatorname{Ker} \varphi$. Logo $M$ é um $R / \partial R$-módulo. Agora será que $\partial(r \circ m)=r \circ(\partial m)$ ? Vejamos: 


$$
\begin{aligned}
\partial(r \circ m) & =\partial\left(r_{(0)} m\right) \\
& \stackrel{(\star)}{=} r_{(0)}(\partial m) \\
& =r(\partial m)
\end{aligned}
$$

onde $(\star)$ vale já que $\partial\left(r_{(0)} m\right)-r_{(0)}(\partial m)=\left[\partial, r_{(0)}\right] m=0$, por $\left(M_{1}\right)$. Logo o 0-ésimo produto comuta com $\partial^{M}$. Agora $\partial(r \circ m)=r \circ(\partial m)$ implica que $\partial M \subset M$ é um submódulo sobre $R / \partial R$, pois $\forall r, r(\partial m)=\partial(r m) \in \partial M$. Logo $M / \partial M$ é um $R / \partial R$-módulo.

Agora suponha que uma superálgebra conforme $R=\underset{i \in I}{\bigoplus} \mathbb{C}[\partial] a^{i}$ seja um $\mathbb{C}[\partial]$-módulo livre e considere a associada superálgebra de Lie de distribuições formais $(\mathfrak{g}(R), F)$ (ver Seção 1). Seja $M$ um módulo sobre a superálgebra conforme $R$ e suponha que $M$ é um $\mathbb{C}[\partial]$-módulo livre com $\mathbb{C}[\partial]$-base $\left\{v^{\alpha}\right\}_{\alpha \in J}$. Este induz um módulo conforme $V(M)$ sobre $\mathfrak{g}(R)$ com elementos de base $v_{(n)}^{\alpha}$, onde $\alpha \in J$ e $n \in \mathbb{Z}$, definido por (confrontar (4.2)):

$$
a^{i}(z) v^{\alpha}(w)=\sum_{j \in \mathbb{Z}_{+}}\left(a_{(j)}^{i} v^{\alpha}\right)(w) \partial_{w}^{(j)} \delta(z-w)
$$

De fato, usando o Teorema 4.8 de forma análoga ao feito na Seção 3, podese associar canonicamente a um módulo conforme $M$ sobre uma superálgebra conforme $R$, um módulo conforme $V(M)$ sobre uma superálgebra de Lie de distribuições formais LieR da seguinte forma:

Primeiro constrói-se o módulo afinizador $\tilde{M}=M\left[t, t^{-1}\right]$ sobre a superálgebra conforme $\tilde{R}$ tomando $\tilde{\partial}^{\tilde{M}}=\partial^{m} \otimes 1+1 \otimes \partial_{t}$ e definindo, para $a \in R, v \in M, f, g \in \mathbb{C}\left[t, t^{-1}\right]$ e $n \in \mathbb{Z}_{+}:$

$$
(a \otimes f)_{(n)}^{M}(v \otimes g)=\sum_{j \in \mathbb{Z}_{+}}\left(a_{(n+j)}^{M} v\right) \otimes\left(\left(\partial_{t}^{(j)} f\right) g\right)
$$

Tem-se que $V(M)=\tilde{M} / \tilde{\partial}^{\tilde{M}} \tilde{M}$, com a ação de LieR induzida pela 0ésima ação:

$$
(a \otimes f)(v \otimes g)=(a \otimes f)_{(0)}^{M}(v \otimes g)
$$

Agora tomando $a_{n}=a \otimes t^{n}$ e $v_{n}=v \otimes t^{n}$, obtem-se de (4.8) uma fórmula explícita da ação de LieR em $V(M)$, com $a \in R, v \in M$ e $m, n \in \mathbb{Z}$ : 


$$
a_{m} v_{n}=\sum_{j \in \mathbb{Z}_{+}}\left(\begin{array}{c}
m \\
j
\end{array}\right)\left(a_{(j)}^{M} v\right)_{m+n-j}
$$

O $($ LieR $R$ )-módulo $(V(M), E(M))$, onde

$$
E(M)=\left\{v(z)=\sum_{n} v_{n} z^{-n-1} / v \in M\right\}
$$

é conforme e $E(M)$ é canonicamente isomorfo ao $R$-módulo $M$. Provas desses fatos são similares aos já feito no Capítulo anterior, sendo desnecessário refazê-los. Agora, como antes, os cálculos ficam bem mais fáceis com a utilização da $\lambda$-ação (onde $a \in R, v \in M$ ):

$$
a_{\lambda}^{M} v=\sum_{n=0}^{\infty} \lambda^{(n)} a_{(n)}^{M} v \in M[[\lambda]]
$$

Assim, os axiomas $\left(M_{1}\right)$ e $\left(M_{2}\right)$ ficam, respectivamente:

$\left(M_{1}\right)_{\lambda}(\partial a)_{\lambda}^{M} v=\left[\partial^{M}, a_{\lambda}^{M}\right] v=-\lambda \cdot a_{\lambda}^{M} v$

$\left(M_{2}\right)_{\lambda}\left[a_{\lambda}^{M}, b_{\mu}^{M}\right] v=\left[a_{\lambda} b\right]_{\lambda+\mu}^{M} v$

Trocando $\mu$ por $\mu-\lambda$ em $\left(M_{2}\right)_{\lambda}$, inverte-se $\left(M_{2}\right)_{\lambda}$ :

$$
\left[a_{\lambda} b\right]_{\mu}^{M} v=\left[a_{\lambda}^{M}, b_{\mu-\lambda}^{M}\right] v
$$

E, desta forma:

$$
\left(a_{(m)} b\right)_{(n)}^{M} v=\sum_{j=0}^{m}(-1)^{m+j}\left(\begin{array}{c}
m \\
j
\end{array}\right)\left[a_{(j)}^{M}, b_{(m+n-j)}^{M}\right] v
$$

De fato, 


$$
\begin{aligned}
& {\left[a_{\lambda}^{M}, b_{\mu-\lambda}^{M}\right]=\sum_{i, j \geq 0} \lambda^{(i)}(\mu-\lambda)^{(j)}\left[a_{(i)}^{M}, b_{(j)}^{M}\right]} \\
& =\sum_{i, j \geq 0} \sum_{k=0}^{j}(-1)^{j-k}\left(\begin{array}{l}
j \\
k
\end{array}\right) \frac{1}{n ! j !} \lambda^{i+j-k} \mu^{k}\left[a_{(i)}^{M}, b_{(j)}^{M}\right] \\
& \stackrel{(\star)}{=} \sum_{m, n \geq 0} \lambda^{(m)} \mu^{(n)} \sum_{i=0}^{m}(-1)^{m-i} \frac{m ! n !}{i !(m+n-i) !}\left(\begin{array}{c}
m+n-i \\
n
\end{array}\right)\left[a_{(i)}^{M}, b_{(m+n-i)}^{M}\right]
\end{aligned}
$$

onde $(\star)$ vale bastando substituir $m$ por $i+j-k$ e $n$ por $k$. Por outro lado,

$$
\begin{aligned}
{\left[a_{\lambda} b\right]_{\mu}^{M} } & =\sum_{n \geq 0} \mu^{(n)}\left[a_{\lambda} b\right]_{(n)}^{M} \\
& =\sum_{n \geq 0} \mu^{(n)}\left(\sum_{m \geq 0} \lambda^{(m)} a_{(m)} b\right)_{(n)}^{M} \\
& =\sum_{m, n \geq 0} \lambda^{(m)} \mu^{(n)}\left(a_{(m)} b\right)_{(n)}^{M}
\end{aligned}
$$

Assim, basta comparar os coeficientes de $\lambda^{(m)} \mu^{(n)}$ para provar (4.12). Seja $R$ uma superálgebra conforme. Pode-se construir um funtor da categoria dos (LieR)-módulos conformes para a categoria dos $R$-módulos conformes levando $(V, E)$ em $M(V)=\bar{E}$ e um funtor no sentido oposto levando $M$ em $(V(M), E(M))$. Como na Seção 3, é fácil ver que estes funtores induzem equivalência entre a categoria das classes de equivalência dos $($ LieR $)$-módulos conformes e a categoria dos $R$-módulos conformes. Toda esta teoria é análoga à já feita na Seção 3 sendo, portanto, desnecessário repetí-lá.

Um módulo conforme $(V, M)$ (respectivamente módulo $M$ ) sobre uma superálgebra de Lie de distribuições formais $(\mathfrak{g}, F)$ (respectivamente sobre uma superálgebra conforme $R$ ) é dito finito se $\bar{E}$ (respectivamente $M$ ) for um $\mathbb{C}[\partial]$-módulo finitamente gerado. Um módulo conforme $(V, M)$ sobre $(\mathfrak{g}, F)$ é chamado irredutível se não existem espaços invariantes não-triviais que contenham todos os $v_{(n)}$, com $n \in \mathbb{Z}$, para algum $v \in \bar{E}$ não-nulo. Obviamente, um módulo conforme é irredutível se, e somente se, o módulo associado $\bar{E}$ sobre a superálgebra conforme $\bar{F}$ for irredutível.

A discussão acima, combinada com o lema abaixo reduz a classificação de módulos conformes finitos sobre uma superálgebra de Lie de distribuições formais $(\mathfrak{g}, F)$ à classificação de módulos finitos sobre a correspondente superálgebra conforme. 
Lema 4.9. (a) Seja $M$ um módulo sobre uma superálgebra conforme $R e$ seja $v \in M$ tal que $\partial v=\lambda \cdot v$, para algum $\lambda \in \mathbb{C}$. Então $v$ é invariante, isto é, $R_{(m)} v=0$, para todo $m \in \mathbb{Z}_{+}$.

(b) Suponha que $M$ seja um módulo finito sobre uma superálgebra conforme e suponha que $M$ não possua invariantes não-nulos. Então $M$ é um $\mathbb{C}[\partial]$ módulo livre.

Demonstração. Seja $a \in R$ e escolha $m$ minimal tal que $a_{(j)} v=0$ para todo $j \geq m$. Temos:

$$
\begin{aligned}
0 & =(\partial-\lambda)\left(a_{(m)} v\right) \\
& =\left[\partial-\lambda, a_{(m)}\right] v+a_{(m)}(\partial-\lambda) v \\
& =-m \cdot a_{(m-1)} v
\end{aligned}
$$

Logo $m=0$, o que prova $(a)$. O ítem $(b)$ segue de $(a)$

Observação 4.10. Dado um módulo $M$ sobre uma superálgebra conforme $R$, pode-se modificar sua estrutura como um $\mathbb{C}[\partial]$-módulo trocando $\partial$ por $\partial+A$, onde $A$ é um endomorfismo sobre $\mathbb{C}$ de $M$ que comuta com todos os $a_{(n)}^{M}$ (isto não afetará o axioma $\left(M_{2}\right)$ ).

Observe que a aplicação $a \rightarrow a_{(n)}$ de $R$ em $\operatorname{End}_{\mathbb{C}} R$ define um $R$-módulo, chamado módulo adjunto.

O exemplo 4.2 nos dá uma família biparametrizada de módulos conformes (irredutíveis sobre a álgebra de Virasoro). Note também que o bemconhecido módulo de Virasoro valuado dado por

$L_{m} v_{(n)}=((\Delta-1)(m+1)-n+\alpha) v_{(m+n)}$

é conforme, mas é finito se, e somente se, $\alpha=0$.

Proposição 4.11. Considere a superálgebra de Lie de distribuições formais $(\mathfrak{g}(R), F)$ definida por:

$\left[a^{i}(z), a^{j}(z)\right]=\sum_{k \in \mathbb{Z}_{+}}\left(a_{(k)}^{i} a^{j}\right)(w) \partial_{w}^{(k)} \delta(z-w)$

e seja $\mathfrak{g}(R)_{+}$a subálgebra aniquiladora de $\mathfrak{g}(R)$ (veja [1] e [3]). Denote por $\mathfrak{g}(R)^{+}$o produto semidireto da subálgebra de Lie unidimensional $\mathbb{C} \partial$ e o ideal $\mathfrak{g}(R)_{+}$com a ação de $\partial$ em $\mathfrak{g}(R)_{+}$dada por $\partial\left(a_{(n)}^{i}\right)=-n \cdot a_{(n-1)}^{i}$. 
Então um módulo $M$ sobre a superálgebra conforme $R$ é, precisamente, um $\mathfrak{g}(R)^{+}$-módulo $M$ (sobre $\mathbb{C}$ ) tal que:

$$
a_{(n)}^{i} v=0
$$

para todo $v \in M$ e $n \gg 0$.

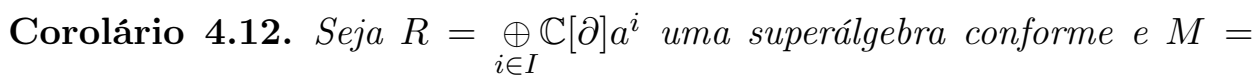
$\sum_{j \in J} \mathbb{C}[\partial] v^{j}$ um $\mathbb{C}[\partial]$-módulo livre. Então dando $a_{(n)}^{i} v^{j} \in M$, para todos os $i \in I, j \in J, n \in \mathbb{Z}_{+}$, e que é zero, para $n \gg 0$, pode-se estender unicamente a ação de $a_{(n)}^{i}$ para todo $R$ em $M$, usando $\left(M_{2}\right)$. Suponha que $\left(M_{1}\right)$ vale para todo $a=a_{(m)}^{i}, b=a_{(n)}^{j}$. Então $M$ é um $R$-módulo.

Usando a Proposição 4.11 e o Corolário 4.12, pode-se construir grandes famílias de módulos finitos sobre superálgebras de Lie de distribuições formais. Na conclusão desta Seção, será listado mais exemplos de módulos sobre superálgebras conformes. Como na Seção 3, os irredutíveis listados abaixo resultam na lista de todos os módulos irredutíveis finitos sobre estas superálgebras conformes.

Exemplo 4.13. Seja $\beta_{0}=\sum_{j \geq 0} \mathbb{C} L_{j}$ e considere uma representação $\pi d a$ álgebra de Lie $\beta_{0}$ num espaço vetorial $U$ de dimensão finita (sobre $\mathbb{C}$ ). Seja $A$ um endomorfismo de $U$ comutando com todo $\pi\left(L_{j}\right)$ (onde $j \in \mathbb{Z}_{+}$). Então $\mathbb{C}[\partial] \otimes U$ é um módulo finito sobre a álgebra conforme $R(\beta)$ definida pelas seguintes fórmulas:

$L_{(0)} u=(\partial+A) u$;

$L_{(j)} u=\pi\left(L_{(j-1)}\right) u$, para $j>1$.

Por exemplo, pode-se tomar $\pi\left(L_{0}\right)=B$, onde $B$ é um endomorfismo de $U$ comutando com A. Tem-se que:

$L_{(0)} u=\partial u+A ;$

$L_{(1)} u=\beta u$;

$L_{(j)} u=0($ para $j>1)$

define um módulo finito sobre a álgebra conforme $R(\beta)$, a qual denota-se por $M_{\beta}(A, B)$. 
Traduzindo isto para a linguagem de superálgebras de Lie de distribuições formais, o exemplo 4.13 cai na seguinte família de módulos conformes finitos sobre a álgebra de Virasoro no espaço $U \otimes \mathbb{C}\left[t, t^{-n-1}\right]$ (lembrando que $\left.u_{(n)}=u \otimes t^{n}\right)$ :

$L_{m} u_{(n)}=(A u)_{(m+n-1)}-(m+n-1) u_{(m+n)}+\sum_{j=0}^{\infty}\left(\begin{array}{c}m+1 \\ j+1\end{array}\right)\left(\pi\left(L_{j}\right) u\right)_{(m+n-j)}$

O caso especial definido para um par $(A, B)$ de operadores comutantes em $U$ é dado por:

$L_{m} u_{(n)}=(A u)_{(m+n-1)}+((m+1) B u-(m+n+1) u)_{(m+n)}$

Usa-se a notação $M_{\beta}^{c}(A, B)$ para este $\beta$-módulo. O superescrito $c$ é para enfatizar que este é um módulo conforme. Será utilizada esta convenção livremente no decorrer do Capítulo. Note que o módulo $M_{\beta}^{c}(A, B)$ é irredutível se, e somente se, o $\beta_{0}$-módulo é irredutível e é trivial se, e somente se, $\operatorname{dim} U=1$ e $\beta=\Delta \neq 0$.

Denota-se estes $\beta$-módulos por $M_{\beta}^{c}(\alpha, \Delta)$, com $\alpha, \Delta \in \mathbb{C}$ e $\Delta \neq 0$. Eles são dados precisamente pelo exemplo 4.2 .

Observação 4.14. Suponha que a subálgebra aniquiladora $\mathfrak{g}_{+} d a$ superálgebra de Lie $\mathfrak{g}^{+}=\mathbb{C}[\partial] \ltimes \mathfrak{g}_{+}$contenha um elemento $L_{-1}$ tal que $a d L_{-1}=\partial$ em $\mathfrak{g}_{+}$. Então $\mathfrak{g}^{+}$é uma soma direta dos ideais $\mathbb{C}\left(\partial-L_{-1}\right)$ e $\mathfrak{g}_{+}$. Portanto, neste caso, o estudo de módulos conformes sobre a correspondente superálgebra conforme (e assim dos módulos conformes) reduz-se ao estudo de módulos sobre $\mathfrak{g}_{+}$satisfazendo (4.13). Esta observação é aplicada a todos os casos, exceto aos das álgebras de Correntes e ao das Supercorrentes.

Exemplo 4.15. Considere a álgebra de Lie de Supercorrente $\tilde{\mathfrak{g}}$ e sua associada superálgebra conforme $R(\tilde{\mathfrak{g}})$. Seja $\pi$ uma representação de $\tilde{\mathfrak{g}}_{+}=$ $\mathfrak{g} \underset{\mathbb{C}}{\otimes} \mathbb{C}[t]$ num espaço vetorial $U$ de dimensão finita tal que $\left(\mathfrak{g} \otimes t^{n}\right) U=0$, para $n \gg 0$. Isto define num espaço $U \otimes \mathbb{C}\left[t, t^{-1}\right]$ a estrutura de um módulo conforme sobre $\tilde{\mathfrak{g}}$ pela fórmula:

$\left(a \otimes t^{m}\right) u_{(n)}=\sum_{j \in \mathbb{Z}_{+}}\left(\begin{array}{c}m \\ j\end{array}\right)\left(\pi\left(a \otimes t^{j}\right) u\right)_{(m+n-j)}$

onde $a \in \mathfrak{g} e u \in U$.

Um caso especial desta construção é quando se toma uma representação $\pi$ 
de dimensão finita da superálgebra de Lie $\mathfrak{g}$ num espaço vetorial de dimensão finita $U$ e estendendo para $\tilde{\mathfrak{g}}_{+}, \operatorname{com} \mathfrak{g} \otimes t \mathbb{C}[t]$ agindo trivialmente. Então tem-se $\left(a \otimes t^{m}\right) u_{(n)}-(\pi(a) u)_{(m+n)}$. Este $\tilde{\mathfrak{g}}$-módulo é denotado por $M_{\tilde{\mathfrak{g}}}^{c}(\pi)$. Traduzindo para a linguagem de módulos sobre a álgebra conforme $R(\tilde{\mathfrak{g}})$, obtem-se o $\mathbb{C}[\partial]$-módulo livre $\mathbb{C}[\partial] \underset{\mathbb{C}}{\otimes} U$, onde:

$a_{(0)} u=\pi(a) u$

$a_{(n)} u=0$, para $n>0$, onde $a \in \mathfrak{g}$ e $u \in U$.

Denota-se este $R(\tilde{\mathfrak{g}})$-módulo por $M_{\tilde{\mathfrak{g}}}(\pi)$. Ele é irredutível se, e somente se, $\pi$ é irredutível. Neste caso, denota-se o módulo por $M_{\tilde{\mathfrak{g}}}(\Lambda)$, onde $\Lambda$ é o peso máximo de $U$.

Exemplo 4.16. Considere a álgebra de Neveu-Schwarz (para $N=1$ ) $\mathfrak{R}$ com superálgebra associada $R(\mathfrak{R})$. Denota-se por $\mathfrak{R}_{+}$a correspondente subálgebra aniquiladora. Considere uma representação de dimensão finita $(\pi, U)$ de $\mathfrak{R}_{0}$, a subálgebra de $\mathfrak{R}_{+}$gerada por elementos "non-negative modes".

Denote por $U^{\theta}$ uma "cópia" de $U$, mas com paridade inversa. Então esta gera uma estrutura de módulo sobre $R(\Re)$ no $\mathbb{C}[\partial]$-módulo livre $\mathbb{C}[\partial] \otimes(U \oplus$ $\left.U^{\theta}\right)$ :

$L_{(0)} u=(\partial+A) u$;

$L_{(j)} u=\pi\left(L_{j-1}\right) u$;

$L_{(0)} u^{\theta}=(\partial+A) u^{\theta}$

$L_{(1)} u^{\theta}=\left(\pi\left(L_{0}\right) u\right)^{\theta}+\frac{1}{2} u^{\theta}$;

$L_{(j+1)} u^{\theta}=\left(\pi\left(L_{j}\right) u\right)^{\theta}+\frac{j+1}{2} \pi\left(G_{j-\frac{1}{2}}\right) u ;$

$G_{(j)} u^{\theta}=\left(\pi\left(G_{j-\frac{1}{2}}\right) u\right)^{\theta}+2 \pi\left(L_{j-1}\right) u$,

onde $j \geq 1, u \in U$, e A é um operador agindo em $U$, comutando com todos os $\pi\left(R_{0}\right)$. Em particular, seja $U=\mathbb{C} u$ o $\mathfrak{R}_{0}$-módulo unidimensional com ação $L_{0} u=\Delta u$, com $0 \neq \Delta \in \mathbb{C}$, e todos os outros geradores agindo trivialmente. Assim, para $A=\alpha \in \mathbb{C}$ arbitrário, obtem-se um módulo irredutivel sobre $R(\Re)$ denotado por $M_{\mathfrak{R}}(\alpha, \Delta)$. Então, como na álgebra de Virasoro, temse uma familia biparametrizada de módulos conformes finitos e irredutiveis denotados por $M_{\mathfrak{R}}^{c}(\alpha, \Delta)$.

Explicitamente, $M_{\mathfrak{R}}^{c}(\alpha, \Delta)=\left(\mathbb{C} u+\mathbb{C} u^{\theta}\right) \underset{\mathbb{C}}{\otimes} \mathbb{C}\left[t, t^{-1}\right]$, com a ação dada 
por:

$L_{m} u_{(n)}=((m+1)(\Delta-1)-n) u_{(n+m)}+\alpha u_{(n+m+1)}$

$L_{m} u_{(n)}^{\theta}=\left((m+1)\left(\Delta-\frac{1}{2}\right)-n\right) u_{(n+m)}^{\theta}+\alpha u_{(n+m+1)}^{\theta} ;$

$G_{r} u_{(n)}=u_{\left(r+n+\frac{1}{2}\right)}$

$G_{r} u_{(n)}^{\theta}=\left(\left(r+\frac{1}{2}\right)(2 \Delta-1)-n\right) u_{\left(r+n-\frac{1}{2}\right)}+\alpha u_{\left(r+n+\frac{1}{2}\right)}$,

onde $u_{(n)}=u \otimes t^{n}, u_{(n)}^{\theta}=u^{\theta} \otimes t^{n}, r \in \frac{1}{2}+\mathbb{Z}$ e $m, n \in \mathbb{Z}$.

Exemplo 4.17. Considere a álgebra supercorrente $\tilde{\mathfrak{g}}_{\text {super. }}$ Seja $R\left(\tilde{\mathfrak{g}}_{\text {super }}\right)$ e $\tilde{\mathfrak{g}}_{\text {super+ }}$ como de costume. Seja $(\pi, U)$ uma representação de dimensão finita de $\tilde{\mathfrak{g}}_{\text {super+ }}$. Obtem-se um módulo sobre $R\left(\tilde{\mathfrak{g}}_{\text {super }}\right)$ como no caso da álgebra corrente, tomando para $n \geq 0$ :

$a_{(n)} u=\pi\left(a \otimes t^{n}\right) u$

$a_{(n)}^{\theta} u=\pi\left(a \otimes t^{n} \theta\right) u$

onde $a \in \mathfrak{g}$ e $u \in U$.

Denota-se estes módulos por $M_{\tilde{\mathfrak{g}}_{\text {super }}}(\pi)$.

No caso especial quando $U$ é uma representação irredutível de dimensão finita de $\mathfrak{g}$ de peso máximo $\Lambda \neq 0$ estendida para $\tilde{\mathfrak{g}}_{\text {super }}+$ trivialmente, o módulo associado sobre $R\left(\tilde{\mathfrak{g}}_{\text {super }}\right)$ será finito e irredutível. Este módulo será denotado por $M_{\tilde{\mathfrak{g}}_{\text {super }}}(\Lambda)$.

O seguinte teorema é imediato da discussão da Seção 2 e do Teorema 3.2 de [1]. Resultados similares vem de todos os outros casos considerados neste texto.

Teorema 4.18. Todo módulo conforme finito, irredutivel e não-trivial sobre a álgebra de Virasoro (respectivamente sobre a álgebra de Supercorrente $\mathfrak{g})$ é isomorfo à $M_{\beta}^{c}(\alpha, \Delta)$ (respectivamente à $M_{\tilde{\mathfrak{g}}}^{c}(\pi)$ ou seus quocientes), onde $\Delta$ é um número complexo não-nulo (respectivamente, $\pi$ é uma representação não-trivial de dimensão finita de $\mathfrak{g})$. 
Para maiores detalhes, veja o teorema 4.36 adiante.

\subsection{Classificação dos módulos conformes finitos irredutíveis}

Seja $(\mathfrak{g}, F)$ uma superálgebra de Lie de distribuições formais. Para todo $N \in \mathbb{Z}_{+}$, seja

$$
\mathfrak{g}_{N}=\sum_{\substack{a \in F \\ n \geq N}} \mathbb{C} a_{(n)}
$$

Suponha que $(\mathfrak{g}, F)$ é regular, isto é, que existe uma derivação $\partial$ de $\mathfrak{g}$ tal que $\partial a_{(n)}=-n \cdot a_{(n-1)}$, para todo $a \in F$ e $n \in \mathbb{Z}$. Obviamente, $(\mathfrak{g}(R), F)$ é regular, onde $R$ é uma superálgebra conforme, daí todos os exemplos considerados antes são regulares. Então $\mathfrak{g}_{+}=\mathfrak{g}_{0}$ é a subálgebra aniquiladora, sendo $\partial$-invariante e, através da Proposição 4.11, tem-se que estudar representações da superálgebra de Lie $\mathfrak{g}^{+}=\mathbb{C} \partial \ltimes \mathfrak{g}_{+}$, conhecida como a subálgebra aniquiladora estendida. Isto leva a considerar a seguinte situação mais abstrata:

Definição 4.19. Seja $\mathfrak{L}$ uma superálgebra de Lie sobre $\mathbb{C}$ com um elemento distinto $\partial$ e uma seqüência de subespaços $\mathfrak{L} \supset \mathfrak{L}_{0} \supset \mathfrak{L}_{1} \supset$.. tais que $\left[\partial, \mathfrak{L}_{k}\right]=\mathfrak{L}_{k-1}$, para todo $k>0$. Seja $V$ um $\mathfrak{L}$-módulo tal que dado $v \in V$, existe um inteiro não-negativo $N$ (dependendo de $v$ ) tal que $\mathfrak{L}_{N} v=0$. Tais $\mathfrak{L}$-módulos serão chamados de $\mathfrak{L}$-módulos conformes. Um $\mathfrak{L}$-módulo conforme é dito finito se for finitamente gerado como um $\mathbb{C}[\partial]$-módulo.

O objetivo principal será descrever $\mathfrak{L}$-módulos conformes finitos irredutíveis. Seja $V$ um $\mathfrak{L}$-módulo conforme, $V_{n}=\left\{v \in V \mid \mathfrak{L}_{n} v=0\right\}$ e $N$ o inteiro não negativo minimal tal que $V_{N} \neq 0$ (que existe por definição). Assim tem-se o lema abaixo:

Lema 4.20. Suponha que $N \geq 1$. Então $\mathbb{C}[\partial] U=\mathbb{C}[\partial] \underset{\mathbb{C}}{\otimes}$ e, além disso, $\mathbb{C}[\partial] U \cap U=U$. Em particular, $U$ é um espaço vetorial de dimensão finita se $V$ for finito.

Demonstração. Denotemos por $L_{a}$ e $R_{a}$ a multiplicação à esquerda e à direita pelo elemento $a$, respectivamente. Usando que $R_{a}=L_{a}-a d a$ e a fórmula binomial, teremos a bem-conhecida fórmula numa álgebra associativa $A$ : 
$\mathfrak{g} a^{k}=\sum_{j=0}^{k}\left(\begin{array}{l}k \\ j\end{array}\right) a^{k-j}(-a d a)^{j} \mathfrak{g}$

com $a, \mathfrak{g} \in A$. Seja $\left\{v_{i}\right\}_{i \in I}$ um conjunto de vetores $\mathbb{C}$-linearmente independentes em $U$ gerando o $\mathbb{C}[\partial]$-módulo $\mathbb{C}[\partial] U$. Suponha que $\sum_{i=1}^{n} p_{i}(\partial) v_{i}=0$, onde $p_{i}(x) \in \mathbb{C}[x]$ e não-todos os $p_{i}(x)=0$. Seja $m$ o grau minimal dos $p_{i}(x)$ 's. Temos que $m \geq 1$, já que $m=0$ implica que $p_{i}(\partial)=p_{i}$ constante, para todo $i$. Assim $\sum_{i=1}^{n} p_{i} v_{i}=0$ implica que $p_{i}=0$, já que $\left\{v_{i}\right\}_{i \in I}$ é l.i.

Escreva $p_{i}(\partial)=\sum_{j=0}^{m} c_{i j} \partial^{j}, \operatorname{com} c_{i j} \in \mathbb{C}$. Então $\exists i$ tal que $c_{i m} \neq 0$, já que $m$ é o grau de pelo menos um deles, por definição. Assim vale, desde que $N \geq 1$ :

$$
\begin{aligned}
\mathfrak{L}_{N+m-1} \partial^{k} & =\sum_{j=0}^{k}\left(\begin{array}{l}
k \\
j
\end{array}\right) \partial^{k-j}(\text { ad } \partial)^{j}\left(\mathfrak{L}_{N+m-1}\right) \\
& =\sum_{j=0}^{k}\left(\begin{array}{l}
k \\
j
\end{array}\right) \partial^{k-j} \mathfrak{L}_{N+m-j-1}
\end{aligned}
$$

já que:

$$
\begin{aligned}
(\text { ad } \partial) \mathfrak{L}_{N+m-1} & =\left[\partial, \mathfrak{L}_{N+m-1}\right] \\
& =\mathfrak{L}_{N+m-2}
\end{aligned}
$$

Portanto: 


$$
\begin{aligned}
0 & =\mathfrak{L}_{N+m-1} v \\
& =\mathfrak{L}_{N+m-1}\left(\sum_{i=1}^{n} p_{i}(\partial) v_{i}\right) \\
& =\sum_{i=1}^{n} \mathfrak{L}_{N+m-1} p_{i}(\partial) v_{i} \\
& =\sum_{i=1}^{N} \mathfrak{L}_{N+m-1} \sum_{j=0}^{m} c_{i j} \partial^{j} v_{i} \\
& =\sum_{i=1}^{n} \sum_{j=0}^{m} c_{i j} \mathfrak{L}_{N+m-1} \partial^{j} v_{i} \\
& =\sum_{i=1}^{n} \sum_{j=0}^{m} c_{i j} \sum_{l=0}^{j}\left(\begin{array}{l}
j \\
l
\end{array}\right) \partial^{j-l} \mathfrak{L}_{N+m-1-l} v_{i}
\end{aligned}
$$

Como toda esta expressão vale zero, então todos seus coeficientes valem zero. Em particular, o 0-ésimo coeficiente (relacionado à 0-ésima potência de $\partial$ ) vale zero e este é obtido em $j=l$. Desta forma:

$\sum_{i=1}^{n} \sum_{j=0}^{m} c_{i j}\left(\begin{array}{l}j \\ j\end{array}\right) \mathfrak{L}_{N+m-1-j} v_{i}=0$

Agora, para todo $i$, temos que $v_{i} \in U=V_{N}=\left\{v \in V \mid \mathfrak{L}_{N} v=0\right\}$. Logo $\mathfrak{L}_{N} v_{i}=0$. Em particular, como $\mathfrak{L}_{N} \supset \mathfrak{L}_{N+m-1-j}$, para todo $j<m$, teremos que $\mathfrak{L}_{N+m-j-1} v_{i}=0$, neste caso. Portanto:

$\sum_{i=1}^{n} c_{i m}\left(\begin{array}{c}m \\ m\end{array}\right) \mathfrak{L}_{N+m-m-1} v_{i}=0$

$\operatorname{logo} \mathfrak{L}_{N-1}\left(\sum_{i=1}^{n} c_{i m} v_{i}\right)=0$, mas existe $i$, com $1 \leq i \leq n$, tal que $c_{i m} \neq 0$, assim $\sum_{i=1}^{n} c_{i m} v_{i} \neq 0$, já que $\left\{v_{i}\right\}_{i \in I}$ é l.i. Portanto, $V_{N-1} \neq(0)$, o que contradiz a minimalidade de $N$. Desta forma todos os $p_{i}(x)$ 's são nulos, o que prova o lema.

Seja $\mathfrak{L} \supset \mathfrak{L}_{0}$ ideal e $V$ um $\mathfrak{L}_{0}$-módulo. Então define-se o módulo induzido $\operatorname{Ind} d_{\mathfrak{L}_{0}}^{\mathfrak{L}} V$ por: 


$$
\operatorname{Ind} d_{\mathfrak{L}_{0}}^{\mathfrak{L}} V=U(\mathfrak{L}) \underset{U\left(\mathfrak{L}_{0}\right)}{\otimes} V
$$

onde $U(\mathfrak{L})$ é a álgebra envolvente universal de $\mathfrak{L}$. Para todo $u \in U(\mathfrak{L})$ e $u^{\prime} \otimes v \in I n d_{\mathfrak{L}_{0}}^{\mathfrak{L}} V$, define-se $u\left(u^{\prime} \otimes v\right)=u u^{\prime} \otimes v$.

De posse disto, tem-se o teorema seguinte:

Teorema 4.21. Assuma que $\left[\mathfrak{L}_{0}, \mathfrak{L}_{i}\right] \subset \mathfrak{L}_{i}$, para todo $i \geq 0$ e que $\mathfrak{L}=$ $\mathbb{C} \partial+\mathbb{C}[\partial, \partial]+\mathfrak{L}_{0}$. Seja $V$ um $\mathfrak{L}$-módulo conforme irredutivel não-trivial. Então ou $V \simeq \operatorname{Ind}_{\mathfrak{L}_{0}}^{\mathfrak{L}} U$, onde $U$ é um $\mathfrak{L}_{0}$-módulo irredutivel (e $\operatorname{dim} U<\infty$ se $V$ for finito), ou então, provado que $\mathfrak{L}_{0}$ é um ideal de $\mathfrak{L}, V$ deve ser um $\mathfrak{L} / \mathfrak{L}_{0}$-módulo irredutivel não-trivial de dimensão finita.

Demonstração. Afirmação 1. $U$ é $\mathfrak{L}_{0}$-invariante.

De fato, $U=V_{N}=\left\{v \in V \mid \mathfrak{L}_{N} v=0\right\}$. Queremos mostrar que $\mathfrak{L}_{0} U \subset U$, isto é, se $v \in U$, então $\mathfrak{L}_{0} v \in U$. Temos que:

$$
\begin{aligned}
\mathfrak{L}_{N}\left(\mathfrak{L}_{0} v\right) & =\left[\mathfrak{L}_{N}, \mathfrak{L}_{0}\right] v+\mathfrak{L}_{0} \mathfrak{L}_{N} v \\
& =0+\mathfrak{L}_{0} 0 \\
& =0
\end{aligned}
$$

já que $\left[\mathfrak{L}_{n}, \mathfrak{L}_{0}\right] \subset \mathfrak{L}_{n}$. Logo $\mathfrak{L}_{0} U \subset U$, e assim, $U$ é $\mathfrak{L}_{0}$-invariante.

Suponha que $N \geq 1$. Então pelo Lema 4.20, vemos que $V$ contém um $\mathfrak{L}$ submódulo isomorfo à $\operatorname{In} d_{\mathfrak{L}_{0}}^{\mathfrak{L}} U$. Logo $V \simeq \operatorname{Ind} d_{\mathfrak{L}_{0}}^{\mathfrak{L}} U$, pois $\mathfrak{L}=\mathbb{C} \partial+\mathbb{C}[\partial, \partial]+\mathfrak{L}_{0}$ e assim, pelo Teorema de Poincaré-Bierhoff-Witt, segue que $U(\mathfrak{L})$ tem uma base de monômios $\partial^{k}[\partial, \partial]^{n} \mathfrak{L}_{0}^{l}$. Desta forma:

$\left\{\partial^{k}[\partial, \partial]^{n} \mathfrak{L}_{0}^{l} \underset{U\left(\mathfrak{L}_{0}\right)}{\otimes} U\right\}=\left\{\partial^{k}[\partial, \partial]^{n} \underset{U\left(\mathfrak{L}_{0}\right)}{\otimes} U\right\} \approx[U(\mathbb{C}(\mathbb{C} \partial) \underset{\mathbb{C}}{\otimes} U(\mathbb{C}[\partial, \partial])] \otimes U$ que:

Logo Ind $d_{\mathfrak{L}_{0}}^{\mathfrak{L}} U=\left(\mathbb{C}[\partial] \underset{\mathbb{C}}{\otimes} \mathbb{C}\left[\partial^{2}\right]\right) \otimes U \approx \mathbb{C}[\partial] \otimes U$. Agora $U \subset V$ implica

$$
\begin{array}{ccc}
U(\mathfrak{L}) & \subset & V \\
\uparrow & & \cup \\
\operatorname{Ind}_{\mathfrak{L}_{0}}^{\mathfrak{L}} U & \simeq & \mathbb{C}[\partial] u
\end{array}
$$


Defina o homomorfismo sobrejetor:

$$
\begin{aligned}
\varphi: U(\mathfrak{L}) \otimes U & \longrightarrow \mathfrak{L} U \\
1 \otimes u & \longmapsto u \\
x \otimes u & \longmapsto x u
\end{aligned}
$$

Assim, Ind $d_{\mathfrak{L}_{0}}^{\mathfrak{L}} U / \operatorname{Ker} \varphi \simeq \operatorname{Im} \varphi=\mathfrak{L} U . \quad$ Logo, $\exists$ um $\mathfrak{L}$-submódulo de $V$ isomorfo a $\operatorname{Ind}_{\mathfrak{L}_{0}}^{\mathfrak{L}} U$. Como $V$ é irredutível, segue que $V \simeq \operatorname{Ind}_{\mathfrak{L}_{0}}^{\mathfrak{L}} U$. Claramente $U$ tem que ser irredutível sobre $\mathfrak{L}_{0}$, já que $V$ é irredutível (pois caso $\exists W \varsubsetneqq U$ submódulo não-nulo, então $\operatorname{Ind}_{\mathfrak{L}_{\mathrm{o}}}^{\mathfrak{L}} W \varsubsetneqq \operatorname{Ind}_{\mathfrak{L}_{0}}^{\mathfrak{L}} U \simeq V$, $\operatorname{logo} V$ seria redutível também). Por outro lado, o mesmo argumento da demonstração do Lema 4.20 mostra que aplicando $\mathfrak{L}_{k}$, para um $k$ conveniente, num elemento não-nulo da forma $\sum_{i=1}^{n} q_{i}(\partial) v_{i}$, onde $q_{i}(\partial) \in \mathbb{C}[\partial]$ e $v_{i} \in U$, obtem-se um elemento não-nulo em $U$. Isto implica que o módulo induzido é irredutível (de fato, $\forall v \neq 0 \in \operatorname{Ind}_{\mathfrak{L}_{0}}^{\mathfrak{L}} V, v=\sum_{i=1}^{n} q_{i}(\partial) v_{i}$. Pelo Lema 4.20, existe $\mathfrak{L}_{k}$ tal que $w=\mathfrak{L}_{k} v \in U$. Como $U$ é irredutível, $w$ gera $U$. Assim $v$ gera todo Ind $d_{\mathfrak{L}_{0}}^{\mathfrak{L}} U$ e, portanto, Ind $d_{\mathfrak{L}_{0}}^{\mathfrak{L}} U$ é irredutível).

Suponha agora que $N=0$. Seja $u$ um vetor não-nulo em $U$. Então $U(\mathfrak{L}) u=\mathbb{C}[\partial] U\left(\mathfrak{L}_{0}\right) u=\mathbb{C}[\partial] u$ e $V=\mathbb{C}[\partial] u$ pela irredutibilidade de $V$ (pois $U(\mathfrak{L}) u=U\left(\mathbb{C} \partial+\mathbb{C}[\partial, \partial]+\mathfrak{L}_{0}\right) u \cong \mathbb{C}[\partial] u \subset V$ e assim $\mathbb{C}[\partial] u=V$, já que $V$ é irredutível). Agora considere 2 casos:

Caso 1. $\mathfrak{L}_{0}$ é um ideal de $\mathfrak{L}$. Então $\mathfrak{L}_{0}$ age trivialmente em $V$ e como $V$ é um $\mathbb{C}[\partial]$-módulo irredutível, segue que se $\partial$ é par, então $V$ será unidimensional e, se $\partial$ é impar com $[\partial, \partial] \neq 0, V$ será então trivial ou um $\mathfrak{L} / \mathfrak{L}_{0}$-módulo bidimensional com $[\partial, \partial]$ agindo como um escalar não-nulo. Para mostrar isto, basta observar que se $\mathfrak{L}_{0}$ é ideal, tem-se $\left[\mathfrak{L}_{\mathfrak{o}}, \partial\right] \subset \mathfrak{L}_{\mathfrak{o}}$. Assim, $\forall v \in V, v=f(\partial) u$ implica que:

$$
\begin{aligned}
\mathfrak{L}_{0} v & =\left[\mathfrak{L}_{0}, f(\partial)\right] u+f(\partial) \mathfrak{L}_{0} u \\
& =0+f(\partial) 0 \\
& =0
\end{aligned}
$$

Logo $\mathfrak{L}_{0}$ age trivialmente em $V$ e $V$ é um $\mathbb{C}[\partial]$-módulo irredutível, pois $V \cong \operatorname{Ind}_{\mathfrak{L}_{0}}^{\mathfrak{L}} U$, que é irredutível aplicando à $\mathfrak{L}_{k}$, com $k=0$. Assim $\forall v \in V, V=U(\mathfrak{L}) v=\mathbb{C}[\partial] U\left(\mathfrak{L}_{0}\right) v$.

Caso 1.1. Se $\partial$ for par, então $[\partial, \partial]=0, \log 0 \mathfrak{L}=\mathbb{C} \partial+\mathfrak{L}_{0}$ (onde $\mathfrak{L}_{0}$ age 
trivialmente em $V$ ). Queremos mostrar que $\operatorname{dim} V=1$. Suponhamos que seja maior do que 1. Então tomando uma base $\beta$ tal que $v_{1}$ seja um autovetor associado a algum autovalor $\lambda \in \mathbb{C}$ (esta base existe pois estamos trabalhando sobre $\mathbb{C}$ ). Temos $\mathbb{C} v_{1}$ é $\partial$-invariante e, portanto, submódulo de $V$, mas $V$ é irredutível, o que é um absurdo. Logo $\operatorname{dim} V=1$ (e $\left.V=\mathbb{C} v_{1}\right)$.

Caso 1.2. Se $\partial$ for ímpar, então temos que $[\partial, \partial]=0$ ou $[\partial, \partial] \neq 0$. Se $[\partial, \partial]=0$, então segue analogamente ao caso 1.1. Então vamos supor que $[\partial, \partial] \neq 0$ e assim $\mathfrak{L}=\mathbb{C} \partial+\mathbb{C}[\partial, \partial]+\mathfrak{L}_{0}$ (onde $\mathfrak{L}_{0}$ age trivialmente em $V$ ). Agora definamos $x=\partial$ e $y=[\partial, \partial]$ (desta forma, $x$ será ímpar e $y$ será par). Temos que $U(\mathbb{C} \partial+\mathbb{C}[\partial, \partial]) \simeq \mathbb{C}[\partial]$. Agora $V=V_{0} \oplus V_{1}$. Se $\operatorname{dim} V_{1}=0$, então $x$ será o operador nulo (já que $x$ é ímpar, logo leva todos os elementos de $V_{0}$ para $V_{1}=\{0\}$ ). Desta forma, $\operatorname{dim} V=1$ e $\mathfrak{L} V=0$ (já que $\partial V=0, \mathfrak{L}_{0} V=0$ e $\partial V=0$ implica que $[\partial, \partial] V=0)$ e assim, $V$ é trivial. Analogamente, se $\operatorname{dim} V_{0} \neq 0$ e $\operatorname{dim} V_{1} \neq 0$, então:

(a) Caso todos os autovalores de $y$ sejam nulos, teremos que $y$ é nilpotente. Agora $x$ e $y$ comutam (pois $[\partial,[\partial, \partial]]=(-1)^{1 \cdot 0}[\partial,[\partial, \partial]]+[[\partial, \partial], \partial]$ pela superidentidade de Jacobi, logo $[[\partial, \partial], \partial]=0, \log 0 x$ e $y$ comutam). Desta forma, eles possuem um autovetor comum. Além disso, $V$ irredutível implica que $V_{0}$ é irredutível sobre $\mathbb{C}[x, y]$, o que implica que $\operatorname{dim} V_{0}=1$ (já que $V_{0}$ possui autovetor $v$ e $\mathbb{C} v$ é submódulo de $V_{0}$ ). Analogamente, $V_{1}$ é irredutível sobre $\mathbb{C}[x, y], \operatorname{logo} \operatorname{dim} V_{1}=1$. Assim $V$ é bidimensional como um $\mathfrak{L} / \mathfrak{L}_{0^{-}}$ módulo. Agora seja $v$ um autovetor comum à $y$ e à $x$. Se $y v=0$, então $x^{2} v=0$. Portanto $\beta \alpha=0$ e, assim, $\beta=0$ ou $\alpha=0$, o que contradiz a irredutibilidade de $V$. Logo $y v \neq 0$. Agora mostremos que $y=[\partial, \partial]$ age como um escalar não-nulo:

$y v=\alpha v, \operatorname{com} \alpha \neq 0$. Será que $y w=\alpha w, \forall w \in V$ ? Vejamos:

Seja $w=x v(=\partial v)$. Temos que $V=\mathbb{C} v \oplus \mathbb{C} w$ como espaço vetorial. Assim (usando o fato de $x$ e $y$ comutarem):

$$
\begin{aligned}
y w & =[\partial, \partial] w \\
& =[\partial, \partial](\partial v) \\
& =\partial([\partial, \partial] v) \\
& =\partial(\alpha v) \\
& =\alpha(\partial v) \\
& =\alpha w
\end{aligned}
$$

Logo, $\forall u \in U$, temos: 


$$
\begin{aligned}
{[\partial, \partial] u } & =[\partial, \partial]\left(u_{v}+u_{w}\right) \\
& =[\partial, \partial] u_{v}+[\partial, \partial] u_{w} \\
& =\alpha u_{v}+\alpha u_{w} \\
& =\alpha u
\end{aligned}
$$

Portanto $[\partial, \partial]$ age como um escalar não-nulo.

Caso 2. Suponha que $\mathfrak{L}_{0}$ seja um ideal de $\mathfrak{L}$. Então existem $l_{0}, l_{0}^{\prime} \in \mathfrak{L}_{0}$ tal que $\left[l_{0}, \partial\right]=\partial+l_{0}^{\prime}$. Um fácil argumento indutivo nos mostra que $l_{0} \partial^{i} u=i \partial^{i} u+f_{i}(\partial) u$, para $i \geq 1$, onde $f_{i}(\partial)$ é um polinômio em $\partial$ de grau no máximo $i-1$ com termo constante nulo.

Agora se $\sum_{i=0}^{n} a_{i} \partial^{i} u$ é um vetor não-nulo de grau no máximo $n-1$. Procedendo assim, vemos que $u$ está contido no módulo gerado por $v$. Então $\partial \mathbb{C}[\partial] \otimes \mathbb{C} u$ é o submódulo maximal de $\mathbb{C}[\partial] \otimes \mathbb{C} u$ e, assim $V$ é o módulo trivial. De fato, $V=\mathbb{C}[\partial] u \nleftarrow \mathbb{C}[\partial] \otimes \mathbb{C} u$, com $\varphi$ homomorfismo sobrejetor. Assim, $\operatorname{Ker} \varphi$ é maximal. Seja $L_{0}=\mathbb{C}[\partial] \otimes \mathbb{C} u$ e seja $L_{1}=\partial \mathbb{C}[\partial] \otimes \mathbb{C} u$. Temos que $L_{1}$ é maximal, pois $L_{0} / L_{1} \cong \mathbb{C} \otimes \mathbb{C} u \cong \mathbb{C} u$, que é irredutível. Além disso, $L_{1}$ é único, pois caso houvesse outro maximal, este teria $a_{0} \neq 0$ e assim, $u$ estaria no módulo gerado por $v\left(a_{0} \neq 0\right.$, pois se $a_{0}=0$, então $L_{1}^{\prime} \subset L_{1}, \operatorname{logo} L_{1}^{\prime}=L_{1}$ ou $L_{1}^{\prime} \subsetneq L_{1} \subsetneq V$, não sendo maximal). Logo:

$$
V \cong L_{0} / L_{1} \cong \mathbb{C} u
$$

Lema 4.22. Seja $\mathfrak{g}$ uma superálgebra de Lie de dimensão finita e seja $\mathfrak{N}$ um ideal solúvel de $\mathfrak{g}$. Seja a uma subálgebra par de $\mathfrak{g}$ tal que $\mathfrak{N}$ é um adamódulo completamente redutivel e não-trivial. Então $\mathfrak{N}$ age trivialmente em todo $\mathfrak{g}$-módulo $V$ irredutivel de dimensão finita.

Antes de demonstrar o lema, o usaremos junto com o teorema 4.21 para provar os corolários abaixo:

Corolário 4.23. Seja $\beta_{+}=\sum_{k \geq-1} \mathbb{C} t^{k+1} \frac{d}{d t}$ a álgebra de Virasoro na linha. Seja $L_{i}=-t^{i+1} \frac{d}{d t}$ e seja $\beta_{0}=\sum_{k \geq 0} \mathbb{C} L_{k}$. Então todo $\beta_{+}$-módulo conforme finito irredutivel não-trivial é da forma $\operatorname{Ind} d_{\beta_{0}}^{\beta_{+}} c_{\lambda}$, onde $c_{\lambda}$ é uma representação irredutivel unidimensional não-trivial de $\beta_{0}, L_{0}$ age como um $\lambda \in \mathbb{C}^{*}$ e $L_{k}$ age como zero, para todo $k>0$. 
Demonstração. Defina $\beta_{i}=\sum_{k \geq i} \mathbb{C} L_{k}$, donde claramente temos que:

$$
\beta \supset \beta_{0} \supset \beta_{1} \supset \ldots
$$

Agora de $\left[L_{n}, L_{m}\right]=(n-m) L_{n+m}$, vem que $\left[\beta_{0}, \beta_{1}\right] \subset \beta_{1}$, já que:

$$
\begin{aligned}
{\left[L_{n}, \beta_{i}\right] } & =\sum_{j \geq 0} \mathbb{C}\left[L_{n}, L_{j}\right] \\
& =\sum_{j \geq 0} \mathbb{C}(n-j) L_{n+j} \subset \beta_{n+j}
\end{aligned}
$$

e, portanto:

$$
\left[\beta_{0}, \beta_{i}\right]=\sum_{l \geq 0} \mathbb{C}\left[L_{j}, \beta_{i}\right] \subset \sum_{l \geq 0} \mathbb{C} \beta_{i+l} \subset \beta_{i}
$$

Defina $\partial=L_{-1}$, onde:

$$
\begin{aligned}
{[\partial, \partial] } & =\left[L_{-1}, L_{-1}\right] \\
& =0
\end{aligned}
$$

Logo:

$$
\begin{aligned}
\beta_{+} & =\mathbb{C} L_{-1}+\sum_{j \geq 0} \mathbb{C} L_{j} \\
& =\mathbb{C} \partial+\mathbb{C}[\partial, \partial]+\beta_{0}
\end{aligned}
$$

Assim, podemos aplicar o Teorema 4.21 e concluir que todo $\beta_{+}$-módulo conforme finito e irredutível não-trivial é da forma $\operatorname{Ind} d_{\beta_{0}}^{\beta_{+}} U$, onde $U$ é um $\beta_{0}$-módulo irredutível finito. Agora $\operatorname{Ind}_{\beta_{0}}^{\beta_{+}} U=U\left(\beta_{+}\right)_{U\left(\beta_{0}\right)}^{\stackrel{\otimes}{\beta_{0}} U} U=U\left(\mathbb{C} L_{-1}+\right.$ $\left.\beta_{0}\right) \otimes U$. Assim, basta analisar $L_{-1} \otimes u$ (já que $U\left(\mathbb{C} L_{-1}\right)$ é uma combinação linear de potências não-negativas de $\left.L_{-1}\right)$, mas antes, temos que:

(a) $U$ é $\beta_{0}$-invariante, $\operatorname{logo} L_{k}: U \rightarrow U, \forall k \geq 0$. Como $U$ é um espaço vetorial sobre $\mathbb{C}$, temos que todo $L_{k}$ admite autovetor. Em particular, $L_{0}$ admite pelo menos um autovetor. Seja $u$ um tal autovetor de $L_{0}$ (isto é, $L_{0} u=\lambda u$, para algum $\left.\lambda \in \mathbb{C}\right)$. Então: 
(b) $\forall k \geq-1$, temos que $L_{k} u$ é um autovetor de $L_{0}$ associado à $\lambda-k$. De fato:

$$
\begin{aligned}
L_{0}\left(L_{k} u\right) & =L_{k} L_{0} u+\left[L_{0}, L_{k}\right] u \\
& =L_{k}(\lambda u)+(0-k) L_{k} U \\
& =(\lambda-k) L_{k} u
\end{aligned}
$$

Agora seja $C=\left\{L_{k} u \mid k \geq 1, u\right.$ é um autovetor qualquer de $\left.L_{0}\right\}$ e seja $U_{1}$ o $\mathbb{C}$-gerado do conjunto $C$. Temos que $U_{1} \subset U$ como espaço vetorial. Vamos provar que $U_{1}$ é $\beta_{0}$-invariante. De fato, $\forall v \in C$ e $j \geq 0$, temos que $L_{j} v \in C$, pois se $j=0$, então $L_{0} v=\alpha v=\alpha L_{k_{c}} u_{c}=L_{k_{c}}\left(\alpha u_{c}\right)$, onde $\alpha u_{c}$ é autovetor de $L_{0}$, já que $u_{c}$ o é e $k_{c} \geq 1$. Se $j \geq 1$, então $v$ é um autovetor (já que $u \in U_{\lambda} \Rightarrow L_{k} u \in U_{\lambda-k}$ ), $\operatorname{logo} L_{j} v \in C$ (pois $j \geq 1$ ). Portanto $c$ é $L_{k}$-invariante, $\forall k \geq 0$ e, desta forma, $U_{1}$ é $L_{k}$-invariante, já que $C$ gera $U_{1}$ e sendo $L_{k}$-invariante, temos que $U_{1}$ é $\beta_{0}$-invariante. Assim $U_{1}$ é submódulo de $U$, que é irredutível. Logo $U=U_{1}$ ou $U=0$. Agora $U \neq U_{1}$, pois na decomposição de $m_{L_{0}}(x)$, posso escolher $\lambda^{\prime}$ tal que sua parte real seja maximal com $w \in U$ tal que $L_{0} w=\lambda^{\prime} w$. Se $w \in U_{1}$, então $\exists k>1$ e $u$ autovetor de $L_{0}$ tal que $w=L_{k} u$, mas isto implica que o autovetor $u$ possui autovalor $\lambda^{\prime}+k$, o que contradiz a maximalidade da parte real de $\lambda$. Desta forma $U \neq U_{1}$ e, portanto, $U_{1}=0$. Assim $c=0$, logo para todo $k \geq 1 \mathrm{e}$ $u \in U$ autovetor, temos que $L_{k} u=0$.

Observação. Por enquanto não provaremos que $L_{k}$, para $k>0$ fixado, age trivialmente em $U$, mas pelo menos já age trivialmente em todos os autovetores de $U$ associados a $L_{0}$. Seja $D=\left\{L_{-1} u \mid u \in\right.$ $U$ é um autovetor qualquer de $\left.L_{0}\right\}$ e seja $U_{2}$ o $\mathbb{C}$-gerado de $D$. Temos que $D$ é $L_{k}$-invariante, pois $L_{-1}\left(L_{-1} u\right)=L_{-1} w$, onde $w=L_{-1} u$ é autovetor de $L_{0}$ (associado à $\left.\beta=\lambda+1\right)$. Agora:

$L_{0}\left(L_{-1} u\right)=\beta L_{-1} u=L_{-1}(\beta u) \in D$

$L_{k}\left(L_{-1} u\right)=0=L_{-1} 0, \forall k>0$, já que $L_{-1} u$ é autovetor de $L_{0}$.

Assim $D$ é $L_{k}$-invariante, $U_{2}$ é $L_{k}$-invariante, portanto $\beta_{+}$-invariante, $\operatorname{logo} U_{2} \subset U$ é submódulo de $U$, sendo então $U_{2}=U$ ou $U_{2}=0$. Mas $U_{2} \neq U$, usando o fato que existe $\lambda \in \mathbb{C}$ com parte real minimal (análogo ao anterior). Portanto $U_{2}=0$ e assim $L_{-1} u=0, \forall u$ autovetor de $L_{0}$. Agora fixemos $u \in U$ autovetor de $L_{0}$. Temos que $U_{3}=\mathbb{C} u$ é $L_{k}$-invariante, para $k \geq-1$. Segue que $\mathbb{C} u$ é $\beta_{0}$-invariante e, desta forma, $U=\mathbb{C} u$.

Logo $U$ é uma representação irredutível unidimensional de $\beta_{0}$. Agora 
suponhamos que $L_{0} u=\lambda u$ é tal que $\lambda=0$. Então $L_{k} u=0, \forall k \geq-1$. Assim $\beta_{+}$age trivialmente em $\mathbb{C} u=U$, mas $V$ é não-trivial. Portanto $\lambda \neq 0 \mathrm{com}$ $L_{0}$ agindo como escalar $\lambda \in \mathbb{C}$ não-nulo e $L_{k}$, para $k \geq 1$, agindo trivialmente em $U$.

Corolário 4.24. Seja $\mathfrak{L}=\beta_{+} \ltimes \mathfrak{g}[t]$ tal que $\left[L_{k}, a \otimes t^{n}\right]=-n \cdot a \otimes t^{n+k}$, com $a \in \mathfrak{g}$. Seja $\mathfrak{L}_{0}=\beta_{0} \ltimes \mathfrak{g}[t]$. Então todo $\mathfrak{L}$-módulo conforme irredutivel finito não-trivial é da forma Ind ${\mathfrak{\mathfrak { L } _ { 0 }}}_{\mathbb{2}} U$, onde $U$ é um $\left(\mathfrak{g} \oplus \mathbb{C} L_{0}\right)$-módulo irredutivel não-trivial com $\mathfrak{g}[t]$ t e $L_{k}$, para $k>0$, agindo trivialmente.

Demonstração. Seja $\mathfrak{L}_{i}=\beta_{i} \ltimes \mathfrak{g}[t]$, onde claramente $\mathfrak{L} \supset \mathfrak{L}_{0} \supset \mathfrak{L}_{1} \supset \ldots$ e seja $\partial=L_{-1}$, onde:

$$
\begin{aligned}
{[\partial, \partial] } & =\left[L_{-1}, L_{-1}\right] \\
& =0
\end{aligned}
$$

Então $\mathfrak{L}=\mathbb{C} \partial+\mathbb{C}[\partial, \partial]+\mathfrak{L}_{0}$ e como $\left[\mathfrak{L}_{0}, \mathfrak{L}_{i}\right] \subset \mathfrak{L}_{i}$, podemos aplicar o teorema 4.21 e concluir que todo $\mathfrak{L}$-módulo conforme finito e irredutível nãotrivial é da forma Ind $d_{\mathfrak{L}_{0}}^{\mathfrak{L}} U$, onde $U$ é um $\mathfrak{L}_{0}$-módulo finito. Falta mostrar que $U$ é, na verdade, um ( $\left.\mathfrak{g} \oplus \mathbb{C} L_{0}\right)$-módulo irredutível não-trivial, mas como

$$
\mathfrak{L}_{0}=\mathbb{C} L_{0}+\mathfrak{g} \otimes 1+\sum_{j \geq 1} \mathbb{C} L_{j}+\mathfrak{g} \otimes \mathbb{C}[t] t
$$

então uma vez provado que $L_{k}$ (para $k \geq 1$ ) e $\mathfrak{g}[t] t$ agem trivialmente em $U$, teremos que $U$ será um $\left(\mathfrak{g}+\mathbb{C} L_{0}+\sum_{j \geq 1} \mathbb{C} L_{k}+\mathfrak{g}[t] t\right)$-módulo e como

$$
\begin{aligned}
{\left[L_{0}, \mathfrak{g}\right] } & =\left[L_{0}, \mathfrak{g} \otimes t^{0}\right] \\
& =-0 \cdot \mathfrak{g} \otimes t^{0+0} \\
& =0
\end{aligned}
$$

a soma será direta e assim $U$ será um $\left(\mathfrak{g} \oplus \mathbb{C} L_{0}\right)$-módulo que será finito e irredutível, já que $V$ o é, e será não-trivial pois $L_{0}$ age de forma nãotrivial. Assim, temos:

(a) Como no Corolário 4.23, existe $u \in U$ e $\lambda \in \mathbb{C}$ tal que $L_{0} u=\lambda u$, onde para $\forall k \geq-1, L_{k} u$ é também autovetor de $L_{0}$, só que associado ao 
autovalor $\lambda-k$. Agora vamos mostrar que para todo $g \in \mathfrak{g}$ e $n \geq 0$, temos que $\left(g \otimes t^{n}\right) u$ é autovetor de $L_{0}$ associado à $\lambda-n$. De fato, temos:

$$
\begin{aligned}
L_{0}\left(a \otimes t^{n} u\right) & =\left[L_{0}, a \otimes t^{n}\right] u+\left(a \otimes t^{n}\right) L_{0} u \\
& =-n \cdot a \otimes t^{n} u+\lambda \cdot a \otimes t^{n} u \\
& =(\lambda-n) \cdot a \otimes t^{n} u
\end{aligned}
$$

Seja $C=\left\{\left(a \otimes t^{n}\right) L_{k}^{m} u \mid u \in U\right.$ é autovetor de $L_{0}, k \geq 1$ e $n \geq 1$ ou $m \geq$ $1\}$. Temos analogamente ao Corolário 4.23 que $C$ é $L_{k}$-invariante, $\forall k \geq 0 \mathrm{e}$ $\mathfrak{g}[t]$-invariante, $\operatorname{logo} U_{1}=\mathbb{C}$-gerado de $C$ é $\mathfrak{L}_{k}$-invariante e $\mathfrak{g}[t]$-invariante. Assim $U_{1} \subset U$ é $\mathfrak{L}_{0}$-invariante e portanto submódulo de $U$ (com $U$ irredutível) sendo $U_{1}=0$ pelo argumento do autovetor $\lambda$ com parte real maximal (ver Corolário 4.23). Desta forma $C=0$ e assim $L_{k} u=0$ e $a \otimes t^{n} u=0$, com $k \geq 1$ e $n \geq 1$, já que pertencem à $C=0$. Seja $B=\left\{u \mid \exists \lambda \in \mathbb{C}\right.$ tal que $\left.L_{0} u=\lambda u\right\}$ e seja $U_{2}$ o $\mathbb{C}$-gerado de $B$. Temos que $B$ é $L_{k}$-invariante, com $k \geq 0$, e é $a \otimes t^{n}$-invariante, com $n \geq 0$. De fato, $\forall v \in B$, temos:

$L_{0} v=\lambda_{v} v \in B$

$L_{k} v=0 \in B$ se $k \geq 1$

$a \otimes t^{n} v \in B$, pois $L_{0}\left(a \otimes t^{n} v\right)=\left(\lambda_{v}-n\right) \cdot a \otimes t^{n} v$

Portanto $U_{2}$ é $L_{k}$-invariante e $a \otimes t^{n}$-invariante. Logo $U_{2}$ é $\mathfrak{g}[t]$-invariante e $\mathfrak{L}_{0}$-invariante e assim, é um submódulo de $U$ irredutível. Assim $U_{2}=U$, já que $U_{2}=0$ não é possível, pois $\exists u$ tal que $L_{0} u=\lambda \cdot u$, para algum $\lambda \in \mathbb{C}$ (pois $\mathbb{C}$ é algebricamente fechado, e assim $m_{\mathfrak{L}_{0}}(x)$ é produto de polinômios de grau 1 possuindo autovetor por esta razão). Assim provamos que existe uma base de autovetores de $U$ e, desta forma, $L_{k}$ e $\mathfrak{g}[t] t$ agem trivialmente em $U$, já que agem trivialmente em todos os elementos de uma base de autovetores de $U$ (lembrando que $\mathfrak{g} \otimes t^{n}$, com $n \geq 1$, gera $\mathfrak{g}[t] t$ ).

Agora $U$ é não-trivial pois, caso contrário, $\mathfrak{L}_{0}$ agiria trivialmente em $U$, $\operatorname{logo} L_{-1} U=0$ e então $\mathfrak{L}$ também agiria trivialmente em $U$, mas

$$
V \simeq \operatorname{Ind} d_{\mathfrak{L}_{0}}^{\mathfrak{L}} U=U(\mathfrak{L})_{U\left(\mathfrak{L}_{0}\right)}^{\otimes} U=0
$$

o que é absurdo, pois $V$ não é trivial.

Corolário 4.25. Seja $R_{+}=\sum_{n \geq-1} \mathbb{C} L_{n}+\sum_{r \geq-\frac{1}{2}} \mathbb{C} G_{r}$ uma álgebra de Neveu- 
Schwarz na linha. Seja $R_{0}=\sum_{n \geq 0} \mathbb{C} L_{n}+\sum_{r>0} \mathbb{C} G_{r}$. Então todo $R_{+}$-módulo conforme finito e irredutivel não-trivial é da forma $\operatorname{Ind}{R_{0}}_{R_{+}} c_{\lambda}$, onde $c_{\lambda}$ é uma representação irregular de $R_{0}, L_{0}$ age como um escalar $\lambda \in \mathbb{C}^{*}$ e $L_{k}$ e $G_{r}$ agem trivialmente para $r, k>0$.

Demonstração. Na álgebra de Neveu-Schwarz, temos:

$\left[L_{m}, L_{n}\right]=(m-n) L_{m+n}$

$\left[G_{r}, L_{n}\right]=\left(r-\frac{n}{2}\right) G_{r+n}$

$\left[G_{r}, G_{s}\right]=2 \cdot L_{r+s}$.

Considere a seguinte filtração:

$R_{2 i}=\sum_{n \geq i} \mathbb{C} L_{n}+\sum_{r \geq i+\frac{1}{2}} \mathbb{C} G_{r}$

Agora $\left[R_{0}, R_{i}\right] \subset R_{i}$, pois $\left[M_{a}, M_{b}\right]=\alpha(a, b) \cdot M_{a+b}$ (onde $M_{a}=L a$ se $a \in \mathbb{Z}, M_{a}=G_{a}$, se $a \in \mathbb{Z}+\frac{1}{2}, \alpha(a, b)=a-b$ se $a, b \in \mathbb{Z}$, etc.). Assim:

$$
\begin{aligned}
{\left[R_{0}, R_{i}\right] } & =\left[\sum_{n \in \frac{1}{2} \mathbb{Z}_{+}} \mathbb{C} M_{n}, \sum_{\substack{m \geq i \\
m \in \frac{1}{2} \mathbb{Z}}} \mathbb{C} M_{m}\right] \\
& =\sum_{\substack{n \geq 0 ; m \geq i \\
n, m \in \frac{1}{2} \mathbb{Z}}} M_{m+n} \subset R_{i}
\end{aligned}
$$

Defina $\partial=G_{-\frac{1}{2}}$, onde $[\partial, \partial]=\left[G_{-\frac{1}{2}}, G_{-\frac{1}{2}}\right]=2 \cdot L_{-1}$. Logo:

$$
\begin{aligned}
R_{+} & =\mathbb{C} G_{-\frac{1}{2}}+\mathbb{C} L_{-1}+R_{0} \\
& =\mathbb{C} \partial+\mathbb{C}[\partial, \partial]+R_{0}
\end{aligned}
$$

Assim, pelo teorema 4.21, temos que todo $R_{+}$-módulo irredutível $V$ é da forma $V \simeq I n d_{R_{0}}^{R_{+}} U$, onde $U$ é um $R_{0}$-módulo irredutível. Agora:

$$
\begin{aligned}
\operatorname{Ind}_{R_{0}}^{R_{+}} U & =U\left(R_{+}\right) \underset{U\left(R_{0}\right)}{\otimes} U \\
& =U\left(\mathbb{C} L_{-1}+\mathbb{C} G_{-\frac{1}{2}}+\beta_{0}\right) \underset{U\left(\beta_{0}\right)}{\otimes} U \\
& =U\left(\mathbb{C} L_{-1} \oplus \mathbb{C} G_{-\frac{1}{2}}\right) \otimes U \\
& =\mathbb{C}\left[L_{-1}\right] \otimes \mathbb{C}\left[G_{-\frac{1}{2}}\right] \otimes U
\end{aligned}
$$


Desta forma, basta analisar elementos da forma $L_{-1} u$ e $G_{-\frac{1}{2}} u$. Sabemos que $\exists u \in U$ e $\lambda \in \mathbb{C}$ tal que $L_{0} u=\lambda u$ e também que $L_{k} u$ é autovetor de $L_{0}$ associado à $(\lambda-k)$. Agora:

$$
\begin{aligned}
L_{0} G_{r} u & =G_{r} L_{0} u+\left[L_{0}, G_{r}\right] u \\
& =\lambda G_{r} u-r G_{r} u \\
& =(\lambda-r) G_{r} u
\end{aligned}
$$

Logo $G_{r} u$ é autovetor de $L_{0}$ associado à $(\lambda-r)$. Por isso, $C=\left\{M_{j>0} u \mid u\right.$ é autovetor de $\left.L_{0}\right\}$ é $M_{j}$-invariante. Logo $U_{1}$ igual ao $\mathbb{C}$-gerado de $L$ é $M_{j}$-invariante, e assim $U_{1}$ é $R_{0}$-invariante e, portanto, submódulo de $U$ irredutível. Mas $U_{1} \neq U$ pelo argumento do $\lambda$ com parte real minimal (ver Corolário 4.23). Assim $U_{1}=0, C=0, L_{k} u=0$ e $G_{r} u=0, \forall k>0, r>0$. Agora seja $D=\left\{u \mid u\right.$ é autovetor de $\left.L_{0}\right\}$ e seja $U_{2}$ o $\mathbb{C}$-gerado de $D$. Temos que $D$ é $L_{k}$-invariante e $G_{r}$-invariante. Portanto, $U_{2}$ é $R_{0}$-invariante e, assim, é submódulo de $U$ irredutível. Logo $U_{2}=U$ (já que $\exists u \in U$ tal que $L_{0} u=\lambda u$, para algum $\lambda \in \mathbb{C}$ ) e como $U$ possui uma base de autovetores tal que $L_{k}$ e $G_{r}$, para $k>0$ e $r>0$ agem trivialmente nesta base, então eles agem trivialmente em $U$.

Agora todos os $\lambda^{\prime} s \neq 0$, pois caso exista $u \in U$ tal que $L_{0} u=0$, teremos que $L_{-1} u=0$ (definindo $A=\left\{L_{-1} v \mid v \in V\right.$ é autovetor de $\left.L_{0}\right\}$ e $V_{1}$ o seu $\mathbb{C}$-gerado. Daí seguirá que $V_{1} \neq V$ pelo argumento do $\lambda$ com parte real minimal), assim teremos que $\mathbb{C} u=V$, já que $V$ é irredutível (e $\mathbb{C} U$ é $\beta_{+^{-}}$ invariante), mas $\beta_{+}$age trivialmente em $\mathbb{C} u=V$, sendo que $V$ é não-trivial por hipótese. $\operatorname{Logo} \lambda \neq 0$. Agora fixado $u \in U$ autovetor de $L_{0}$, temos que $U_{3}=\mathbb{C} U$ é $L_{k}$-invariante para $k \geq 0$. Portanto $U_{3}$ é $\beta_{0}$-invariante e assim $U=\mathbb{C} u$. Logo $U$ é uma representação irredutível $c_{\lambda}$ de $\beta_{0}$.

Observação 4.26. Traduzindo a linguagem de módulos sobre a subálgebra aniquiladora $\beta_{+} \ltimes \mathfrak{g}[t]$ do Corolário 4.24 para a linguagem de módulos sobre álgebras conformes, obtêm-se uma família triparametrizada de módulos nãotriviais sobre $R(\beta) \ltimes R(\tilde{\mathfrak{g}})$. Denota-se estes módulos por $M_{\beta \ltimes \tilde{\mathfrak{g}}}(\Lambda, \alpha, \Delta)$, onde $\Lambda$ é um peso integral dominante. Claramente, quando $\Lambda \neq 0, M_{\beta \ltimes \tilde{\mathfrak{g}}}(\Lambda, \alpha, \Delta)$ é irredutivel. Quando $\Lambda=0, M_{\beta \ltimes \tilde{\mathfrak{g}}}(0, \alpha, \Delta)$, para $\Delta \neq 0$, é irredutivel.

Assim esgota-se todos os casos de módulos finitos sobre $R(\beta) \ltimes R(\tilde{\mathfrak{g}})$. O correspondente módulo conforme sobre $\beta \ltimes \tilde{\mathfrak{g}}$ é irredutível e esgota todos os casos de módulos conformes irredutíveis finitos não-triviais. Usando 
filtrações similares às do corolário 4.25, tem-se os corolários 4.27 e 4.28 adiante:

Corolário 4.27. Seja $\mathfrak{g}$ uma soma direta de superálgebras de Lie simples de dimensão finita. Seja $\mathfrak{L}=\left(\mathbb{C} \frac{\partial}{\partial t}+\mathbb{C}\left(\theta \frac{\partial}{\partial t}-\frac{\partial}{\partial \theta}\right)\right) \ltimes \mathfrak{g}[t, \theta]$. Então todo $\mathfrak{L}$-módulo conforme finito e irredutivel não-trivial ou é da forma Ind $d_{\mathfrak{g}[t, \theta]}^{\mathfrak{L}} U$, onde $U$ é uma representação irredutivel de dimensão finita de $\mathfrak{g}$ ou é uma representação irredutivel bidimensional da subálgebra $\mathbb{C} \frac{\partial}{\partial t}+\mathbb{C}\left(\theta \frac{\partial}{\partial t}-\frac{\partial}{\partial \theta}\right)$, onde $\frac{\partial}{\partial t}$ age como um escalar não-nulo e $\mathfrak{g}[t, \theta]$ age trivialmente.

Demonstração. Seja $\mathfrak{L}_{2 i}=t^{i} \mathfrak{g}[t, \theta]$ e $\mathfrak{L}_{2 i+1}=t^{i} \theta \mathfrak{g}[t, \theta]$, para $i \in \mathbb{N}$, onde claramente $\left[\mathfrak{L}_{0}, \mathfrak{L}_{i}\right] \subset \mathfrak{L}_{i}$. Defina:

$$
\partial=\theta \frac{\partial}{\partial t}-\frac{\partial}{\partial \theta}
$$

Temos que $\theta, \frac{\partial}{\partial \theta}$ são ímpares, enquanto que $\frac{\partial}{\partial t}$ é par e:

$$
\begin{aligned}
{[\partial, \partial] } & =\left[\theta \frac{\partial}{\partial t}-\frac{\partial}{\partial \theta}, \theta \frac{\partial}{\partial t}-\frac{\partial}{\partial \theta}\right] \\
& =2 \cdot\left[\theta \frac{\partial}{\partial t},-\frac{\partial}{\partial \theta}\right] \\
& =2 \cdot\left(\theta \frac{\partial}{\partial t}\left(-\frac{\partial}{\partial \theta}\right)+\frac{\partial}{\partial \theta} \theta \frac{\partial}{\partial t}\right) \\
& =2 \cdot \frac{\partial}{\partial t}\left(-\theta \frac{\partial}{\partial \theta}+\frac{\partial}{\partial \theta} \theta\right) \\
& =2 \cdot \frac{\partial}{\partial t}
\end{aligned}
$$

Desta forma, $\mathfrak{L}=\mathbb{C} \partial+\mathbb{C}[\partial, \partial]+\mathfrak{L}_{0}$ e, portanto, podemos aplicar o Teorema 4.21 e concluir que:

(a) Se $N \geq 1$, então $V \simeq \operatorname{Ind}_{\mathfrak{g}[t, \theta]}^{\mathfrak{L}} U$, onde $U$ é uma representação irredutível de dimensão finita.

(b) Se $N=0$, estaremos na mesma situação do caso (1) da demonstração do Teorema 4.21, onde $\partial$ é ímpar e $[\partial, \partial] \neq 0$ e, como $V$ não é trivial, então $V$ é um $\mathfrak{L} / \mathfrak{L}_{0}$-módulo bidimensional (lembrando que $\mathfrak{L} / \mathfrak{L}_{0} \simeq \mathbb{C} \partial+\mathbb{C}[\partial, \partial]=$ $\left.\mathbb{C}\left(\theta \frac{\partial}{\partial t}-\frac{\partial}{\partial \theta}\right)+\mathbb{C} \frac{\partial}{\partial t}\right)$, onde $[\partial, \partial]=2 \cdot \frac{\partial}{\partial t}$ age como um escalar não-nulo e 
$\mathfrak{g}[t, \theta]$ age trivialmente.

Corolário 4.28. Seja $\mathfrak{L}=R_{+} \ltimes \mathfrak{g}[t, \theta]$. Seja $\mathfrak{L}_{0}=R_{0} \ltimes \mathfrak{g}[t, \theta]$. Então todo $\mathfrak{L}$-módulo conforme finito irredutivel não-trivial é da forma $\operatorname{Ind}_{\mathfrak{L}_{0}}^{\mathfrak{L}} U$, onde $U$ é um $\left(\mathfrak{g} \oplus \mathbb{C} L_{0}\right)$-módulo de dimensão finita tal que $L_{k}$ e $G_{r}$, para todos os $k, r>0, e \mathfrak{g}[t] t+\mathfrak{g}[t] \theta$ agindo trivialmente.

Demonstração. Temos que:

$$
\begin{aligned}
L_{n} & =-t^{n}\left(t \frac{\partial}{\partial t}+\frac{n+1}{2} \theta \frac{\partial}{\partial \theta}\right) \\
G_{r} & =-t^{r+\frac{1}{2}}\left(\theta \frac{\partial}{\partial t}-\frac{\partial}{\partial \theta}\right)
\end{aligned}
$$

Assim, $L_{-1}=\frac{\partial}{\partial t}$ e $G_{-\frac{1}{2}}=\theta \frac{\partial}{\partial \theta}-\frac{\partial}{\partial \theta}$ como no Corolário anterior. Lembrando que $\theta^{2}=0$, temos:

(a) $\left[L_{k}, a \otimes t^{n}\right]=a \otimes L_{k} t^{n}$

$$
\begin{aligned}
& =a \otimes\left(-t^{k}\left(t \cdot n \cdot t^{n-1}\right)\right) \\
& =-n \cdot a \otimes t^{n+k}
\end{aligned}
$$

(b) $\left[L_{k}, a \otimes T^{n} \theta\right]=a \otimes L_{k} t^{n} \theta$

$$
\begin{aligned}
& =a \otimes\left(-t^{k}\left(t \cdot n \cdot t^{n-1} \theta+\frac{k+1}{2} t^{n} \theta\right)\right) \\
& =-\left(n+\frac{k+1}{2}\right) a \otimes t^{n+k} \theta
\end{aligned}
$$

(c) $\left[G_{r}, a \otimes t^{n}\right]=a \otimes G_{r} t^{n}$

$$
\begin{aligned}
& =a \otimes\left(-t^{r+\frac{1}{2}}\left(\theta \cdot n \cdot t^{n-1}\right)\right) \\
& =-n \cdot a \otimes t^{n+r-\frac{1}{2}} \theta
\end{aligned}
$$

$(d)\left[G_{r}, a \otimes t^{n} \theta\right] a \otimes G_{r} t^{n} \theta=a \otimes\left(-t^{r+\frac{1}{2}}\left(n \cdot t^{n-1} \theta^{2}-t^{n}\right)\right)$

$$
=a \otimes t^{n+r+\frac{1}{2}}
$$

Usando a filtração $\mathfrak{L}_{i}=R_{i} \ltimes \mathfrak{g}[t, \theta]$ (ver Corolário 4.26), temos que:

$$
\begin{aligned}
\mathfrak{L} & =\mathbb{C} G_{-\frac{1}{2}}+\mathbb{C} L_{-1}+\mathfrak{L}_{0} \\
& =\mathbb{C} \partial+\mathbb{C}[\partial, \partial]+\mathfrak{L}_{0}
\end{aligned}
$$


e também que $\left[\mathfrak{L}_{0}, \mathfrak{L}_{i}\right] \subset \mathfrak{L}_{i}$. Logo podemos aplicar o Teorema $4.21 \mathrm{e}$ concluir que todo $\mathfrak{L}$-módulo conforme finito e irredutível não-trivial é da forma $\operatorname{Ind} d_{\mathfrak{L}_{0}}^{\mathfrak{L}} U$, onde $U$ é um $\mathfrak{L}_{0}$-módulo finito. Falta mostrar que $U$ é, na verdade, um $\left(\mathfrak{g} \oplus \mathbb{C} L_{0}\right)$-módulo irredutível não-trivial, mas como

$$
\mathfrak{L}_{0}=\mathbb{C} L_{0}+\mathfrak{g} \otimes 1+\sum_{k \geq 1} \mathbb{C} L_{k}+\sum_{r \geq \frac{1}{2}} \mathbb{C} G_{r}+\mathfrak{g} \otimes \mathbb{C}[t] t+\mathfrak{g} \otimes \mathbb{C}[t] \theta
$$

então uma vez provado que $L_{k}$ e $G_{r}$ agem trivialmente em $U$, para $r, k>0$, e também que $\mathfrak{g}[t] t$ e $\mathfrak{g}[t] \theta$ agem trivialmente, teremos que esta soma será direta. Agora $U$ é finito e irredutível pois $V$ é finito e irredutível e é nãotrivial pois $L_{0}$ age de forma não-trivial. Assim, temos:

(a) Como no Corolário 4.24, existe $u \in U$ e $\lambda \in \mathbb{C}$ tal que $L_{0} u=\lambda u$, donde para todo $k \geq-1, L_{k} u$ é também autovetor de $L_{0}$, só que associado ao autovalor $\lambda-k$ e $\left(\mathfrak{g} \otimes t^{n}\right) u$ é autovetor associado ao autovalor $\lambda-n$ (como no Corolário 4.25). Agora analisemos $G_{r} u$ e $\left(a \otimes t^{n} \theta\right) u$ :

$$
\begin{aligned}
& \begin{aligned}
L_{0} G_{r} u & =\left[L_{0}, G_{r}\right] u+G_{r} L_{0} u \\
= & -r \cdot G_{r} u+\lambda G_{r} u \\
= & (\lambda-r) G_{r} u
\end{aligned} \\
& \begin{aligned}
L_{0} a \otimes t^{n} \theta u & =\left[L_{0}, a \otimes t^{n} \theta\right] u+a \otimes t^{n} \theta L_{0} u \\
& =-\left(n+\frac{1}{2}\right) a \otimes t^{n} \theta u+\lambda a \otimes t^{n} \theta u \\
& =\left(\lambda-n-\frac{1}{2}\right) a \otimes t^{n} \theta u
\end{aligned}
\end{aligned}
$$

Seja $C=\left\{\left(a \otimes t^{n} \theta^{i} L_{k}^{\theta} G_{r}^{p}\right) u \mid i \in\{0,1\}, u \in U\right.$ é um autovetor de $L_{0} ; k, r>0 ; a \neq 0$ e $(n \geq 1$ ou $i=1$ ou $\theta \geq 1$ ou $\left.p \geq 1)\right\}$ e seja $U_{1}$ o $\mathbb{C}$-gerado de $C$. De forma análoga ao do Corolário 4.25, temos que $U_{1}=0$, $C=0, L_{k} u=0, G_{r} u=0, a \otimes t^{n} u=0($ se $n \geq 1)$ e $a \otimes t^{n} \theta u=0($ se $n \geq 0)$, para todo $u$ autovetor de $L_{0}$. Agora seja $B=\left\{u \mid \exists \lambda \in \mathbb{C}\right.$ tal que $\left.L_{0} u=\lambda u\right\}$ e seja $U_{2}$ o seu $\mathbb{C}$-gerado. Temos que $B$ é $\left(L_{k}, G_{r}, a \otimes t^{n} \theta^{i}\right)$-invariante, logo $U_{2}$ também o é. Assim $U_{2}$ é $\mathfrak{L}_{0}$-invariante e, portanto, $U_{2}$ é submódulo de $U$ irredutível. Como $U_{2} \neq 0$ (já que $\exists u \in U$ e $\exists \lambda \in \mathbb{C}$ tal que $L_{0} u=\lambda u$ ), temos que $U_{2}=U$. Assim, provamos que existe uma base de autovetores de 
$U$ e, desta forma, $L_{k}, G_{r}, \mathfrak{g}[t] t$ e $\mathfrak{g}[t] \theta$ agem, de fato, trivialmente em todo $U$, já que o fazem numa base de $U$.

Agora feitos os corolários acima, demonstremos o Lema 4.22:

Demonstração. Primeiro observemos que se $[a, b]=b$, então $b$ é nilpotente em qualquer representação de dimensão finita $V$. De fato, se $v \in V$ é um autovetor de $a$ com autovalor $\lambda$, então:

$$
\begin{aligned}
b v & =[a, b] v \\
& =a(b v)-(-1)^{p(a) p(b)} b(a v) \\
& =a(b v)-b(\lambda v) \\
& =a(b v)-\lambda b v
\end{aligned}
$$

Logo $a(b v)=b v+\lambda b v=(\lambda+1) b v$. Desta forma, $b^{n} v$ é um autovetor de $a$ associado a $\lambda+n$. Se, por absurdo, $b^{n} v \neq 0, \forall n \in \mathbb{N}$, então $\left\{b^{i} v\right\}_{i \in \mathbb{N}}$ seria, via Álgebra Linear, um conjunto l.i., já que são autovetores associados a autovalores 2 a 2 disjuntos. Assim $\operatorname{dim} V=+\infty$ (um absurdo). Logo $\exists n \geq 1$ tal que $b^{n-1} v \neq 0$, mas $b^{n} v=0$. Portanto $\exists w \in V$, com $w$ não-nulo, tal que $b w=0$. Seja $W$ o espaço aniquilado por $b(W \neq 0$, pois $w \in W)$. Então $W$ é $a$-invariante já que $\forall w \in W, b(a(w))=[a, b] w+a b(w)=b w+a b w=$ $0+a \cdot 0=0, \operatorname{logo} a W \subset W$, o que prova que $W$ é $a$-invariante. Agora como $W \neq 0$, então $\operatorname{dim} V / W<\operatorname{dim} V$. Assim:

Passo 1: Se $\operatorname{dim} V=1$, então $V=\mathbb{C} v_{1}, \operatorname{logo} a\left(v_{1}\right)=\lambda v_{1}$, pois $a\left(v_{1}\right) \in V$. Agora $b\left(v_{1}\right)$ é autovetor associado a $\lambda+1, \operatorname{logo}\left\{v_{1}, b\left(v_{1}\right)\right\}$ é l.i. se $b\left(v_{1}\right) \neq 0$, mas isto contradiz $\operatorname{dim} V=1$. Logo $b\left(v_{1}\right)=0$ e assim $b$ é nilpotente.

Passo 2: Suponhamos que vale caso $\operatorname{dim} V<n$ e provemos que vale se $\operatorname{dim} V=n$. De fato, se $\operatorname{dim} V=n$ e $W \neq 0$, então $\operatorname{dim} V / W<n$. Por hipótese de indução, $b$ é nilpotente em $V / W$. Agora mostremos que $\exists n_{1}>0$ tal que $\forall v \in V, b^{n_{1}}(v)=0$. Sabemos que $\exists n^{\prime}$ tal que $b^{n^{\prime}}(\bar{v})=\overline{0} \in V / W$, $\operatorname{com} \bar{v} \in V / W$. Logo $b^{n^{\prime}}(v)=w \in W$. Assim $b^{n^{\prime}+1}(v)=b(w)=0$, logo basta considerar $n=n^{\prime}+1$.

Agora seja $\mathfrak{s}$ um ada-submódulo irredutível de $n$. Por hipótese, este é um módulo com peso máximo não-nulo. Portanto, existem $a \in \mathfrak{a}, b \in \mathfrak{s}$ tais que $[a, b]=b, \operatorname{logo} b$ é nilpotente. Além disso, todos os elementos da órbita $A d \mathfrak{a} \cdot b$, onde $A$ é o grupo de Lie conectado à álgebra de Lie $\mathfrak{a}$, é nilpotente em $V$. Assim, $\mathfrak{s}$ é $A d A$-irredutível, sua órbita gera $\mathfrak{s}$ e, portanto, $\mathfrak{s}$ é gerado 
pelos elementos que são nilpotentes em $V$. Então todo $a$-submódulo de $\mathfrak{n}$ é gerado pelos elementos que são nilpotentes em $V$. De fato, sabemos que:

1) $\mathfrak{n}$ é um ideal solúvel de $\mathfrak{g}$.

2) $\mathfrak{g}=\mathfrak{g}_{0} \oplus \mathfrak{g}_{1}$, com $a \subset \mathfrak{g}_{0}$ tal que $[a, \mathfrak{n}] \subset \mathfrak{n}$

3) $\mathfrak{n}$ é um ada-módulo completamente redutível com somandos não-triviais (ou seja, $\mathfrak{n}=V_{1} \oplus \ldots \oplus V_{p}$, com $\operatorname{dim} V_{i}>1$ e $V_{i}$ irredutíveis).

4) $\mathfrak{s}$ é um ada-submódulo irredutível $\left(\operatorname{logo} s=V_{i}\right.$ para algum $\left.i, 1 \leq i \leq p\right)$, o que implica que este é um módulo com peso máximo não-nulo (ver página 285 de [14], teorema 11.5), isto é,

$\mathfrak{s} \approx V(\lambda), \operatorname{com} \lambda \in H^{*}, \lambda \neq 0($ se $\lambda=0$, então $\operatorname{dim} V(\lambda)=1)$

5) $\forall a \in \mathfrak{a}, a \neq 0, a: \mathfrak{s} \rightarrow \mathfrak{s}$. Como estamos sobre $\mathbb{C}, a$ tem autovetores. Seja $b$ um autovetor com autovalor $u$.

Defina $a^{\prime}=\frac{1}{u} a$ e, desta forma, $a^{\prime}(b)=b$. Assim, definindo $a d a(b)=$ $[a, b]=a^{\prime}(b)$, teremos $b$ nilpotente em $V$. Para provar o Lema, assuma que $V$ é um g-módulo fiel (lembrando que $\rho: \mathfrak{g} \rightarrow g l(V)$ é dita fiel se $\operatorname{Ker} \rho=0$. Assim podemos supor, sem perda de generalidade, que $V$ é um módulo fiel, bastando considerar $V / K \operatorname{er} \rho$ caso não seja). Suponhamos que o Lema não é verdadeiro, isto é, $\mathfrak{n}$ é não-nulo. Seja $\mathfrak{n}^{(i)}$ o último elemento não-nulo de sua série derivada. Pelo acima, $\mathfrak{n}^{(i)}$ é gerada por elementos mutuamente comutativos que são nilpotentes em $V$, e então $\mathfrak{n}^{(i)}$ aniquila um vetor não-nulo em $V$. De fato, suponhamos que $\mathfrak{n}$ é não-nulo. Seja $\mathfrak{n}^{(i)} \neq 0$ tal que $\mathfrak{n}^{(i+1)}=\left[\mathfrak{n}^{(i)}, \mathfrak{n}^{(i)}\right]=0$. Temos que $\mathfrak{n}^{(i)}$ é gerado por elementos mutuamente comutativos que são nilpotentes em $V$, pois $\forall a, b \in \mathfrak{n}^{(i)},[a, b]=a b-b a \in \mathfrak{n}^{(i+1)}=0, \operatorname{logo} a b=b a$ sendo, portanto, comutativos. Agora estes são nilpotentes em $V$ pois toda órbita $A d A \cdot b$ é nilpotente em $V$. Agora

$$
\left\{v \in V / \mathfrak{n}^{(i)} v=0\right\}=V^{\prime} \subset V, V^{\prime} \neq 0
$$

Assim, $\forall x \in \mathfrak{g}, \mathfrak{n}^{(i)} x v=0 \Rightarrow x v \in V^{\prime}, \forall v \in V^{\prime}$. Agora $\mathfrak{n}^{(i)}=$ $\operatorname{span}\left\{u_{1}, \ldots, u_{k}\right\}$. Como $u_{i}$ e $u_{j}$ comutam $\forall i, j$ e todos possuem autovetores associados ao 0 , então existe $w$ não-nulo em $V$ tal que $\mathfrak{n}^{(i)} w=0$. Mas $\mathfrak{n}^{(i)}$ é um ideal de $\mathfrak{g}$ e, portanto, o subespaço de $V$ aniquilado por $\mathfrak{n}^{(i)}$ é um $\mathfrak{g}$-submódulo de $V, \operatorname{logo} \mathfrak{n}^{(i)}$ é um $\mathfrak{g}$-submódulo de $V$, mas $V$ é irredutível. Assim $\mathfrak{n}^{(i)}$ aniquila $V$ e, desta forma, $V$ não é fiel (um absurdo). 
Observação 4.29. O Corolário 4.28 nos dá uma família triparametrizada de módulos finitos irredutiveis não-triviais sobre a superálgebra conforme $R(\mathfrak{R}) \ltimes R\left(\hat{\mathfrak{g}}_{\text {super }}\right)$. Denotemos estes módulos por $M_{R \ltimes \tilde{\mathfrak{g}}_{\text {super }}}(\Lambda, \alpha, \Delta)$, onde $\Lambda$ é um peso inteiro dominante de $\mathfrak{g} e \alpha, \Delta \in \mathbb{C}$. As condições para irredutibilidade desses módulos e suas classificações são como na Observação 4.26. O mesmo vale para os correspondentes módulos conformes sobre $R \ltimes \tilde{\mathfrak{g}}_{\text {super }}$.

O Lema 4.22, contudo, não é aplicável à álgebra de Corrente de séries triplas de superálgebras de Lie simples, chamadas de superálgebras de Lie $A(m \mid n)$, para $m \neq n, c(n)$ e $w(n)$ (veja [5]), para o qual $n$ contém somandos não-triviais. Para estas 3 séries, os enunciados do corolário 3.1 e do teorema 3.2 de [3] estão incorretos. Abaixo classificaremos os módulos conformes finitos e irredutíveis sobre suas álgebras de Correntes, mas antes apresentaremos uma solução para $A(m \mid n)$, já que o Lema 4.22 não se aplica a este caso. Assim, é suficiente considerar representações irredutíveis de dimensão finita das superálgebras de Lie $\mathfrak{g} \otimes \mathbb{C}[t] / t^{n+1}$, onde $n \geq 0$ e $\mathfrak{g}$ é um termo de uma das três séries de superálgebras mencionadas. Como o caso de $n=0$ é trivial, podemos assumir que $n \geq 1$. De fato, estudaremos mais geralmente as superálgebras de Lie $\mathfrak{g}$ que satisfazem propriedades que descreveremos agora.

Seja $\mathfrak{g}=\mathfrak{g}_{\overline{0}} \oplus \mathfrak{g}_{\overline{1}}$ uma superálgebra de Lie de dimensão finita e suponha que $\mathfrak{g}=\mathfrak{g}_{-1} \oplus \mathfrak{g}_{0} \oplus \mathfrak{g}_{1} \oplus \ldots \oplus \mathfrak{g}_{r}$ é $\mathbb{Z}$-graduado tal que $\mathfrak{g}_{i} \subseteq \mathfrak{g}_{i}$. Assuma que $\mathfrak{g}_{0}=a \oplus \mathbb{C} c$ é uma álgebra de Lie redutível tal que $a$ é uma subálgebra semisimples e $c$ é um elemento central. Além disso, suponha que $\mathfrak{g}_{i}$ é um $a$-módulo com somandos não-triviais para $i \neq 0$ e que existe um contragradiente $a$-submódulos $\mathfrak{g}_{-1}^{*} \subseteq \mathfrak{g}_{1}$ e $\mathfrak{g}_{1}=\mathfrak{g}_{-1}^{*} \oplus \mathfrak{g}_{1}^{\prime}$ como um $a$-módulo, com $\left[\mathfrak{g}_{1}^{\prime}, \mathfrak{g}_{-1}\right] \subseteq a$. Finalmente, suponha dado algum $a \in \mathfrak{g}_{-1}$ não-nulo, com $\left[a, \mathfrak{g}_{-1}^{*}\right]$ tendo projeção não-trivial em $\mathbb{C} c$ e dado qualquer $b \in \mathfrak{g}_{-1}^{*}$ não-nulo, $\left[\mathfrak{g}_{-1}, b\right]$ tendo projeção não-trivial em $\mathbb{C} c$. Note que isto segue da descrição das séries triplas de superálgebras de Lie simples $A(m \mid n)$, para $m \neq n, c(n)$ e $w(n)$ em [5], satisfazendo as hipóteses de $\mathfrak{g}$ abaixo:

Seja $\mathfrak{L}=\mathfrak{g} \otimes \mathbb{C}[t] /\left(t^{n+1}\right)$. Queremos determinar $\mathfrak{L}$-módulos irredutíveis de dimensão finita, com o qual $\mathfrak{g} \otimes t^{n}$ age de modo não-trivial. Temos:

$$
G_{0}=\mathfrak{g}_{0}+\mathfrak{g}_{1}^{\prime}+\sum_{i \geq 2} \mathfrak{g}_{i}
$$

Considere a subálgebra $L \subseteq \mathfrak{L}$, que é definida da seguinte forma: 
(a) Se $n=2 k$, temos:

$L=G_{0}+G_{0} \otimes t+\ldots+G_{0} \otimes t^{k-1}+\left(G_{0}+\mathfrak{g}\right) \otimes t^{k}+\mathfrak{g} \otimes t^{k+1}+\ldots+\mathfrak{g} \otimes t^{2 k}$

(b) Se $n=2 k+1$, então temos:

$$
L=G_{0}+G_{0} \otimes t+\ldots+G_{0} \otimes t^{k}+\mathfrak{g} \otimes t^{k+1}+\ldots+\mathfrak{g} \otimes t^{2 k+1}
$$

Determinaremos primeiro os $L$-módulos irredutíveis de dimensão finita. Isto nos mostrará que todo $L$-módulo irredutível com $\mathfrak{g} \otimes t^{n}$ agindo nãotrivialmente é obtido por indução num determinado $L$-módulo irredutível. A principal ferramenta que usaremos para provar esta asserção é o análogo algébrico de Lie do critério de irredutibilidade de Mackey (ver [7]). Depois precisaremos de algumas terminologias.

Seja $e$ uma superálgebra de Lie de dimensão finita e $I \subseteq e$ um ideal de $e$. Seja $\left(\pi, V_{I}\right)$ um $I$-módulo irredutível e defina o estabilizador associado ao par $(\pi, I)$ sendo $K_{\pi}=\left\{k \in e \mid \exists A_{k} \in \operatorname{End}\left(V_{I}\right), \operatorname{com} \pi([k, i])=\left[A_{k}, \pi(i)\right], \forall i \in\right.$ $I\}$. Agora $K_{\pi}$ é uma subálgebra central contendo $I$. Podemos provar isto tudo usando argumentos análogos aos de [7]. Para o teorema abaixo, veja [8].

Teorema 4.30. Seja $V_{k}$ uma representação irredutivel de $K_{\pi}$ tal que como um $I$-módulo, $V_{k}$ é uma soma direta de cópias de $\pi$. Se o espaço $\mathbb{Z}_{2}$-graduado

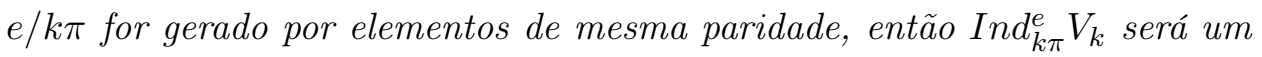
e-módulo irredutivel.

Consideraremos agora a subálgebra de Borel de $\mathfrak{g}_{0}$. Então $B$ é uma subálgebra solúvel de $L$ e $\left[B_{\overline{1}}, B_{\overline{1}}\right]$ tem projeção trivial em $\mathbb{C} c$. Contudo, como $\mathfrak{g}$ (visto como um $a$-módulo) contém apenas somandos triviais, chamados $\mathbb{C} c$, é claro que $\left[b, \mathfrak{g}_{-1}^{*}\right]=\mathfrak{g}_{-1}^{*}$, para todo $i \neq 0$, e $\left[b, \mathfrak{g}_{0}\right]=a$. De fato, $\left[b, \mathfrak{g}_{-1}^{*}\right]=$ $\mathfrak{g}_{-1}^{*}$ e também $\left[b, \mathfrak{g}_{1}^{\prime}\right]=\mathfrak{g}_{1}^{\prime}$. Esta discussão implica que $\left[B_{\overline{1}}, B_{\overline{1}}\right] \subseteq\left[B_{\overline{0}}, B_{\overline{0}}\right]$. Assim como em [5], toda representação irredutível de dimensão finita de $B$ é unidimensional, chamada $\mathbb{C} v_{\lambda}$, onde $\lambda: h+\mathbb{C} c \otimes \mathbb{C}[t] \rightarrow \mathbb{C}$ é uma forma linear estendida trivialmente para $B$ e $h$ é uma subálgebra de Cartan em $b$. Contudo, $I n d_{\beta}^{L} \mathbb{C} v_{\lambda}$ é gerado pelos elementos da forma $e_{-\alpha_{1}}^{k \alpha_{1}} \ldots e_{-\alpha_{m}}^{k \alpha_{m}} v_{\lambda}$, onde $e_{-\alpha_{j}}$ são vetores de origem negativa de $a$. Segue que $c \otimes t^{j}$ age como um escalar em $\operatorname{Ind} d_{\beta}^{L} \mathbb{C} v_{\lambda}$. Temos $\mathfrak{g}^{c}=\mathfrak{g}_{-1}+a+\sum_{i \geq 1} \mathfrak{g}_{i}$.

Claramente, $\left[a, \mathfrak{g}^{c}\right]=\mathfrak{g}^{c}$. Então segue por indução em $k \alpha_{1}+\ldots+k \alpha_{m}$ que $\mathfrak{g}_{1}^{\prime}+\sum_{i \geq 2} \mathfrak{g}_{i}+\left(\mathfrak{g}^{c} \otimes \mathbb{C}[t] t\right) \cap L$ age trivialmente em seu módulo induzido. 
Então temos:

Proposição 4.31. Todo L-módulo irredutivel é um $\mathfrak{g}_{0} \oplus(c \otimes \mathbb{C}[t] t)$-módulo irredutivel, onde $\mathfrak{g}_{1}^{\prime}+\sum_{i \geq 2} \mathfrak{g}_{i}+\left(\mathfrak{g}^{c} \otimes \mathbb{C}[t] t\right) \cap L$ age trivialmente.

Agora introduziremos a subálgebra auxiliar $\overline{\mathfrak{L}}=G_{0}+\mathfrak{g} \otimes \mathbb{C}[t]$ t. Pela Proposição 4.31, obtemos a classificação de $\overline{\mathfrak{L}}$-módulos irredutíveis de dimensão finita.

Proposição 4.32. Seja $V_{L}$ um L-módulo irredutível tal que $c \otimes t^{n}$ age como um escalar não-nulo. Então Ind $d_{L}^{\overline{\mathfrak{I}}} V_{L}$ é irredutivel. Além disso, suponha que $w$ é um $\overline{\mathfrak{L}}$-módulo irredutível com $\mathfrak{g} \otimes t^{n}$ agindo não-trivialmente, então $W \simeq \operatorname{Ind} d_{L}^{\overline{\mathfrak{s}}} V_{L}$, onde $V_{L}$ é como antes.

Demonstração. Seja $I=\left(G_{0}+\mathfrak{g}_{1}\right) \otimes t^{k}+\mathfrak{g} \otimes \mathbb{C}[t] t^{k+1}$, se $n=2 k$ ou $I=\mathfrak{g} \otimes \mathbb{C}[t] t^{K+1}$, se $n=2 k+1$. Note que $I$ é um ideal de $\overline{\mathfrak{L}}$. Pela Proposição 4.31, $V_{L}$ é uma soma direta de $I$-módulos unidimensionais mutuamente isomorfos $\mathbb{C} v_{\lambda}$, onde $\left(c \otimes t^{j}\right) v_{\lambda}=\lambda\left(c \otimes t^{j}\right) v_{\lambda}$. Considere o estabilizador do $I$-módulo $\mathbb{C} v_{\lambda}$ em $\overline{\mathfrak{L}}$. Como para todo vetor $\mathfrak{g} \in \mathfrak{g}_{-1}+\mathfrak{g}_{-1}^{*}$, existe um vetor $\mathfrak{g}^{\prime} \in \mathfrak{g}_{-1}+\mathfrak{g}_{-1}^{*}$ tal que $\left[\mathfrak{g}, \mathfrak{g}^{\prime}\right]=u c$, onde $u \neq 0$ e $a \in a+\mathfrak{g}_{2}$. Segue que o estabilizador é exatamente $L$, se $\lambda\left(c \otimes t^{n}\right) \neq 0$. Como $\overline{\mathfrak{L}} / L$ é também um espaço vetorial par completo, podemos utilizar o Teorema $4.30 \mathrm{e}$ a irredutibilidade seguirá. Por outro lado, suponha que $w$ seja um $\overline{\mathfrak{L}}$-módulo irredutível com o qual $\mathfrak{g} \otimes t^{n}$ age não-trivialmente. Seja $V_{L}$ um $L$-submódulo irredutível sobre $w$. Se $c \otimes t^{n}$ age trivialmente em $V_{L}$, então $\mathfrak{g} \otimes t^{n}$ age nãotrivialmente em $V_{L}$ pela Proposição 4.31. Contudo, $\mathfrak{g} \otimes t^{n}$ é um ideal de $\overline{\mathfrak{L}}$ e, portanto, age trivialmente em $W$, o que é uma contradição.

A Proposição 4.31 nos diz que quando induzimos de $L$ para $\overline{\mathfrak{L}}$, a irredutibilidade é preservada. Agora nosso principal objetivo é demonstrar por indução que $\overline{\mathfrak{L}}$ para $\mathfrak{L}$ também preserva irredutibilidade. Note que, da Proposição 4.32, temos:

Corolário 4.33. Todo $\overline{\mathfrak{L}}$-módulo irredutivel, onde $c \otimes t^{n}$ age nãotrivialmente, considerado como um $\mathfrak{g} \otimes t^{n}$-módulo, é uma soma direta dos mesmos $\mathfrak{g} \otimes t^{n}$-módulos unidimensionais $\mathbb{C} v_{\lambda}$. Além disso, $c \otimes t^{n}$ age como um escalar não-nulo $\lambda\left(c \otimes t^{n}\right)$, onde $\mathfrak{g}^{c} \otimes t^{n}$ age trivialmente.

Agora estamos próximos de classificar $\mathfrak{L}$-módulos irredutíveis. 
Proposição 4.34. Seja $V$ um $\overline{\mathfrak{L}}$-módulo irredutível, onde $c \otimes t^{n}$ age como um escalar não-nulo. Então Ind $\mathfrak{\mathfrak { L }}_{\mathfrak{L}}^{\mathfrak{L}} V$ é um $\mathfrak{L}$-módulo irredutivel. Além disso, todo $\mathfrak{L}$-módulo irredutivel, com $\mathfrak{g} \otimes t^{n}$ agindo não-trivialmente, é desta forma.

Demonstração. Iremos utilizar o Teorema 4.30 agora. Pelo Corolário 4.33, $V$ é uma soma direta de cópias dos $\mathfrak{g} \otimes t^{n}$-módulos $\mathbb{C} v_{\lambda}$ e $\lambda\left(c \otimes t^{n}\right) \neq 0$. Usando o mesmo argumento que o da Prova da Proposição 4.32, podemos verificar que o estabilizador é precisamente $\overline{\mathfrak{g}}$ se $\lambda\left(c \otimes t^{n}\right) \neq 0$. Então pelo Teorema 4.30, o módulo induzido é irredutível. O mesmo argumento da prova da Proposição 4.32 nos diz que todos são irredutíveis.

As Proposições 4.32 e 4.34 juntas nos dão:

Teorema 4.35. Todas as representações irredutiveis de dimensão finita de $\mathfrak{L}$, onde $\mathfrak{g} \otimes t^{n}$ age não-trivialmente, são da forma $\operatorname{Ind} d_{L}^{\mathfrak{L}} V_{L}$, onde $V_{L}$ é uma representação irredutivel de $L$, onde $c \otimes t^{n}$ age como um escalar não-nulo. Além disso, todas estas representações são irredutiveis.

Em [8], prova-se que se $S$ é uma superálgebra de Lie de dimensão finita tal que $[S, S]=S$ e $\Lambda(n)$ é a superálgebra de Grassman em $n$ variáveis ímpares, então todo $S \otimes \Lambda(n)$-módulo é um $S$-módulo irredutível, onde $S \otimes N$ age trivialmente, sendo $N$ o ideal nilpotente maximal de $\Lambda(n)$. Então para as supercorrentes $\mathfrak{g}[t, \theta]=\mathfrak{g} \otimes \mathbb{C}\left[t, t^{-1}\right] \otimes \Lambda(1)$, onde $\theta$ é uma variável ímpar e $\mathfrak{g}$ é uma das três séries de superálgebras de Lie simples, podemos aplicar estes resultados e o Teorema 4.35 para obter seus módulos conformes irredutíveis. Em vista a tudo isto, temos:

Resultado Principal: Relacionemos $R(\beta), R(\tilde{\mathfrak{g}}), R(\Re)$ e $R\left(\hat{\mathfrak{g}}_{\text {super }}\right)$ com as álgebras de Virasoro, de Corrente, de Neveu-Schwarz e a conforme supercorrente, respectivamente. Denotemos $R(\beta) \ltimes R(\tilde{\mathfrak{g}})$ e $R\left(\mathfrak{R} \ltimes R\left(\tilde{\mathfrak{g}}_{\text {super }}\right)\right.$ suas respectivas somas semidiretas. Então o seguinte é uma lista completa de seus módulos finitos irredutíveis não-unidimensionais (sobre $\mathbb{C}$ ):

(1) $M_{\beta}(\alpha, \Delta)$ e $M_{\Re}(\alpha, \Delta)$, onde $\alpha, \Delta \in \mathbb{C}$, com $\Delta \neq 0$.

(2) $M_{\tilde{\mathfrak{g}}}(\Lambda)$ e $M_{\tilde{\mathfrak{g}}_{\text {super }}}(\Lambda)$, onde $\Lambda$ é um peso inteiro dominante não-nulo.

(3) $M_{\beta \ltimes \tilde{\mathfrak{g}}}(\Lambda, \alpha, \Delta)$ e $M_{\beta \ltimes \mathfrak{g}_{\text {super }}}(\Lambda, \alpha, \Delta)$, onde $\Lambda$ é um peso inteiro dominante não-nulo e $\alpha, \Delta \in \mathbb{C}$, ou então se $\Lambda=0$, temos $\Delta \neq 0$ (relembrar os exemplos 4.13 e 4.15$)$. 
Para mostrar este resultado, temos o teorema abaixo:

Teorema 4.36. Seja $R$ uma álgebra conforme de um dos três tipos descritos no final da Seção 3.2. Então a lista completa dos R-módulos conformes finitos, irredutiveis, não-triviais $M$ é a seguinte:

(a) Se $R$ é uma álgebra conforme de Corrente Curg onde $\mathfrak{g}$ é uma álgebra de Lie semisimples de dimensão finita, então $M \cong M(\tilde{U})$, onde $U$ é um $\mathfrak{g}$-módulo irredutível não-trivial de dimensão finita.

(b) Se $R$ for a álgebra conforme de Virasoro, então $M \cong M(\Delta, \alpha)$, com $\Delta \neq 0$.

(c) Se $R$ for a soma semidireta da álgebra conforme de Virasoro e da álgebra conforme de Corrente Cur $\mathfrak{g}$, onde $\mathfrak{g}$ é uma álgebra de Lie de dimensão finita, então $M \cong M(\Delta, \alpha, U)$, onde $U$ é um $\mathfrak{g}$-módulo irredutivel de dimensão finita que deverá ser não-trivial se $\Delta=0$.

Demonstração. (a) Seja $R=$ Curg. Então temos:

$$
\mathfrak{L}=(\text { LieR })^{-}=\mathbb{C} T+\mathfrak{g}[T], T=-\partial_{t}
$$

com a filtração $\mathfrak{L}_{n}=\mathfrak{g}[t] t^{n}, n \in \mathbb{Z}_{+}$. Seja $M$ um $R$-módulo irredutível. Então este é um $\mathfrak{L}$-módulo conforme e podemos aplicar o Lema 4.19. Se $N \geq 1$, temos que $M=\mathbb{C}[T]_{\mathbb{C}}^{\otimes} M_{N}$, onde $M_{n}$ é um $\mathfrak{L}_{0} / \mathfrak{L}_{N}$-módulo irredutível. Se, além disso, $M$ é um módulo finito, então $\operatorname{dim}_{\mathbb{C}} M_{N}<\infty$ e poderemos aplicar um resultado bem-conhecido da teoria das Álgebras de Lie que diz que $M_{N}$ é um $\mathfrak{g}[t]$-módulo irredutível com ação trivial de $\mathfrak{g}[t] t$.

Se $N=0$, então $M_{N}$ é um $\mathfrak{g}[t]$-módulo trivial, logo um $\mathfrak{L}$-submódulo, e $\operatorname{assim} M=M_{N}$ é um $R$-módulo trivial.

(b) Seja Vir a álgebra conforme de Virasoro. Então temos $(\text { Lie Vir })^{-}=$ $\mathbb{C} T+$ Vect $\mathbb{C}$, onde Vect $\mathbb{C}=\bigoplus_{n \in \mathbb{Z}_{+}} c t^{n} \partial_{t}$, e $T$ age neste como $-a d \partial_{t}$. Isto mostra que (Lie Vir) ${ }^{-}$é uma soma direta (como ideais) da álgebra de Lie comutativa $\mathbb{C}\left(T+\partial_{t}\right)$ e a álgebra de $\mathfrak{L}=V$ ect $\mathbb{C}$.

Seja $M$ um Vir-módulo irredutível. Este é um (Lie Vir $)^{-}$-módulo irredutível. Logo $T+\partial_{t}$ age como um escalar, que denotaremos por $\alpha$ e 
$\mathfrak{L}$ age irredutivelmente em $M$. Defina a seguinte filtração em $\mathfrak{L}:$

$$
\mathfrak{L}_{n}=\bigoplus_{j \geq n} \mathbb{C} t^{j+1} \partial_{t}
$$

e apliquemos o Lema 4.19.

Se $N \geq 1$, argumentamos da mesma forma que no ítem $(a)$ para mostrar que $M=\mathbb{C}[T] \underset{\mathbb{C}}{\otimes} M_{N} \operatorname{com} M_{N}$ irredutível, e para mostrar que $M \cong M(\Delta, \alpha)$, com $\Delta \neq 0$, se $M$ for finito. Se $N=0$, então é fácil ver que $M$ é um $\mathfrak{L}$ módulo trivial unidimensional. A prova de $(c)$ é análoga.

Agora provaremos o seguinte teorema:

Teorema 4.37 (Análogo Conforme do Teorema de Lie). Seja M um módulo conforme finito sobre uma álgebra conforme finita e solúvel $R$. Então existe um autovetor comum (com autovalores em $\mathbb{C}$ ) de todos os operadores $a_{(n)}^{M}$, onde $a \in R, n \in \mathbb{Z}_{+}$.

Demonstração. Provaremos este teorema por indução na ordem lexicográfica do par (postoR, dim tor $R$ ) de inteiros não-negativos. Seja $S \subset R$ o último membro não-nulo de uma série derivada de $R$. Então $S$ é comutativo e $R_{(j)} S \subset S$, para todo $j \in \mathbb{Z}_{+}$. Logo temos uma representação de $R / S$ em $S$. Pela indutividade desta aplicação à álgebra conforme $R / S$, temos que existe um elemento não-nulo $b \in S$ tal que

$$
R_{(j)} b \in \mathbb{C} b
$$

para $j \in \mathbb{Z}_{+}$.

De fato, consideremos os operadores de álgebras de Lie em $M$

$$
\begin{aligned}
\mathfrak{g} & =(\text { LieR })_{-}^{M}=\sum_{\substack{a \in R \\
j \in \mathbb{Z}_{+}}} \mathbb{C} a_{(j)}^{M} \\
b & =\mathbb{C} \partial^{M}+\sum_{j \in \mathbb{Z}_{+}} \mathbb{C} b_{(j)}^{M}
\end{aligned}
$$


com a filtração $b_{n}=\sum_{j \geq n} \mathbb{C} b_{(j)}^{M}$. Renomeando

$$
\left[a_{(m)}^{M}, b_{(n)}^{M}\right]=\sum_{j \geq 0}\left(\begin{array}{c}
m \\
j
\end{array}\right)\left(a_{(j)} b\right)_{(m+n-j)}^{M}
$$

temos que

$$
\left[\mathfrak{g}, b_{n}\right] \subset b_{n}
$$

Seja $M_{n}=\left\{v \in M / b_{n} v=0\right\}$ e seja $N$ o menor $n \in \mathbb{Z}_{+}$tal que $M_{n} \neq 0$.

Caso 1: $N=0$

Então segue de (4.15) que $M_{0}$ é um $R$-submódulo não-nulo, logo um $R / \mathbb{C}[\partial] R$-submódulo, e podemos aplicar a indução sugerida.

Caso 2: $N \geq 1$

Então pelo Lema 4.19 aplicado ao $b$-módulo $M$, $\operatorname{dim}_{\mathbb{C}} M_{N}<\infty$. Por (4.15), $\mathfrak{g} M_{N} \subset M_{N}$ e desde que $\mathfrak{g}$ é uma álgebra de Lie solúvel, podemos aplicar o Teorema de Lie clássico para encontrar um autovetor para $\mathfrak{g}$ em $M_{N}$.

Observação 4.38. O teorema 4.36 nos diz que a família $M_{\mathfrak{R}}^{c}(\alpha, \Delta)$ (ver exemplo 4.16 para fórmulas explícitas) esgota todos os casos de módulos conformes irredutíveis não-triviais sobre a álgebra de NeveuSchwarz. Similarmente, o mesmo teorema também classifica todos os módulos conformes irredutíveis não-triviais de somas semidiretas.

Como na demonstração em [8], todas as álgebras conformes finitas semisimples são uma soma de álgebras da forma $R(\beta), R(\tilde{\mathfrak{g}})$ e $R(\beta) \ltimes R(\tilde{\mathfrak{g}})$. Os resultados desta seção nos dão uma descrição de todos os módulos irredutíveis finitos sobre todas as álgebras conformes finitas semisimples. A saber, temos o seguinte:

Proposição 4.39. Seja $R=R_{1} \oplus R_{2} \oplus \ldots \oplus R_{n}$ uma álgebra conforme semisimples finita. Suponha que $R_{i}$ é ou $R(\beta)$ ou $R(\beta) \ltimes R(\tilde{\mathfrak{g}})$, para algum 
i. Seja $V$ um módulo irredutivel finito da álgebra conforme $R$ com restrição a $R_{i}$ não-trivial. Então a restrição de $V$ a todos os $R_{j}$ são não-triviais, para $i \neq j$.

Demonstração. Como $R_{i}$ é uma das álgebras conformes $R(\beta)$ ou $R(\beta) \ltimes R(\tilde{\mathfrak{g}})$, existem $L_{(0)}^{i} \in R_{i}$ tais que $\left[\partial-L_{(0)}^{i}, R_{i}\right]=0$. Escolha qualquer $k \neq i$ e considere módulos irredutíveis sobre a álgebra conforme $R_{i} \oplus R_{k}$. Pela Proposição 4.11, considere módulos sobre $\mathbb{C} \partial \ltimes\left(\left(R_{i}\right)_{+} \oplus\left(R_{k}\right)_{+}\right) \simeq\left(R_{i}\right)_{+} \oplus$ $\left(\mathbb{C}\left(\partial-L_{(0)}^{i}\right) \ltimes\left(R_{k}\right)_{+}\right)$.

Agora um $\left(R_{i}\right)_{+}$-módulo irredutível não-trivial é livre sobre $\mathbb{C}\left[L_{(0)}^{i}\right]$ e também um $\mathbb{C}\left(\partial-L_{(0)}^{i}\right) \ltimes\left(R_{k}\right)_{+}$-módulo irredutível não-trivial é livre sobre $\mathbb{C}\left[\partial-L_{(0)}^{i}\right]$ pelo já discutido. Então nosso produto tensorial é livre sobre $\mathbb{C}\left[L_{(0)}^{i}\right] \otimes \mathbb{C}\left[\partial-L_{(0)}^{i}\right]$ e, portanto, não pode ser finito sobre $\mathbb{C}[\partial]$.

A Proposição 4.39 e o Teorema 4.36 implicam:

Teorema 4.40. Seja $R=R_{1} \oplus R_{2} \oplus \ldots \oplus R_{n}$ uma álgebra conforme finita semisimples, onde todo $R_{i}$ ou é simples ou da forma $R(\beta) \ltimes R(\tilde{\mathfrak{g}})$. Então $R$ tem um módulo finito fiel, se e somente se, todos os $R_{i}$ 's são álgebras conformes de Correntes, ou $n=1$. 


\section{Referências Bibliográficas}

[1] KAC,V.G., Vertex Algebra for begginers, University Lecture Notes, Vol. 10, AMS, Providence, 1996.

[2] LEPOWSKY,J.; LI,H., Introduction to Vertex Operator Algebras and Their Representations, Progress in Mathematics., Vol. 227

[3] CHEnG, S.J.; KAC, V.G., Conformal Modules, Asian J. Math., Vol.1, $N_{\circ} 1$ (1997) 181-193

[4] D'ANDREA,A.; KAC,V.G., Structure theory of finite conformal algebras, Selecta Math. (1998)

[5] KAC, V.G., Lie Superalgebras, Adv. in Math., 26 (1977) 8-96.

[6] BORCHERDS, R., Vertex algebras, Kac-Moody algebras, and the Monster, Proc. Natl. Acad. Sci. USA, 83, (1986), 3068-3071.

[7] BLATTNER, R.J., Induced and Produced Modules of Lie Algebras, Trans. AMS, 144 (1969) 457-474.

[8] CHENG, S.J., Differentiably Simple Lie Superalgebras and Representations of Semisimple Lie superalgebras, J. Alg., 173 (1995) $1-43$.

[9] KAC,V.G., Simple irreducible graded Lie algebras of finite growth., Math. USSR-Izvestija 2 (1968), 1271-1311.

[10] MATHIEU, O., Sur un problème de V.G.Kac: la classificacion de certaines algèbres de Lie graduèes simples. J. Algebra 102 (1986), 505536 .

[11] MATHIEU, O., Classification des algèbres de Lie graduèes simples de croissance $\leq 1$., Invent. Math. 86 (1986), 371-426

[12] MATHIEU, O., Classification of simple graded Lie algebras of finite growth., Invent. Math. 108 (1992), 455-519. 
[13] GELFAND,I.M., DORFMAN, I.Ya., Hamiltonian operators and algebraic structures related to them, Funct. Anal. and Appl. 13 (1979), 248-262.

[14] SAN MARTIN, L.A.B, Álgebras de Lie, Editora Unicamp 